\title{
A Neural Network Approach to the Virtual Dressing Room
}

By

Thanasiri Muttulingam, B. Eng

Bachelors of Engineering, Carleton University, 2005

A thesis submitted to

The faculty of Graduate Studies and Research

In partial fulfillment of

The requirements for the degree of

\section{Master of Applied Science in Systems and Computer Engineering}

\author{
Ottawa-Carleton Institute for Electrical and Computer Engineering \\ Department of Systems and Computer Engineering \\ Carleton University \\ Ottawa, Ontario
}

\author{
CCopyright \\ 2007, Thanasiri Muttulingam
}




$\begin{array}{ll}\begin{array}{l}\text { Library and } \\ \text { Archives Canada }\end{array} & \begin{array}{l}\text { Bibliothèque et } \\ \text { Archives Canada }\end{array} \\ \begin{array}{l}\text { Published Heritage } \\ \text { Branch }\end{array} & \begin{array}{l}\text { Direction du } \\ \text { Patrimoine de l'édition }\end{array} \\ \begin{array}{l}\text { 395 Wellington Street } \\ \text { Ottawa ON K1A ON4 }\end{array} & \begin{array}{l}\text { 395, rue Wellington } \\ \text { Ottawa ON K1A ON4 } \\ \text { Canada }\end{array}\end{array}$

Your file Votre référence ISBN: 978-0-494-33662-5 Our file Notre référence ISBN: 978-0-494-33662-5

NOTICE:

The author has granted a nonexclusive license allowing Library and Archives Canada to reproduce, publish, archive, preserve, conserve, communicate to the public by telecommunication or on the Internet, loan, distribute and sell theses worldwide, for commercial or noncommercial purposes, in microform, paper, electronic and/or any other formats.

The author retains copyright ownership and moral rights in this thesis. Neither the thesis nor substantial extracts from it may be printed or otherwise reproduced without the author's permission.
AVIS:

L'auteur a accordé une licence non exclusive permettant à la Bibliothèque et Archives Canada de reproduire, publier, archiver, sauvegarder, conserver, transmettre au public par télécommunication ou par l'Internet, prêter, distribuer et vendre des thèses partout dans le monde, à des fins commerciales ou autres, sur support microforme, papier, électronique et/ou autres formats.

L'auteur conserve la propriété du droit d'auteur et des droits moraux qui protège cette thèse. $\mathrm{Ni}$ la thèse ni des extraits substantiels de celle-ci ne doivent être imprimés ou autrement reproduits sans son autorisation.
In compliance with the Canadian

Privacy Act some supporting forms may have been removed from this thesis.

While these forms may be included in the document page count, their removal does not represent any loss of content from the thesis.
Conformément à la loi canadienne sur la protection de la vie privée, quelques formulaires secondaires ont été enlevés de cette thèse.

Bien que ces formulaires aient inclus dans la pagination, il n'y aura aucun contenu manquant.

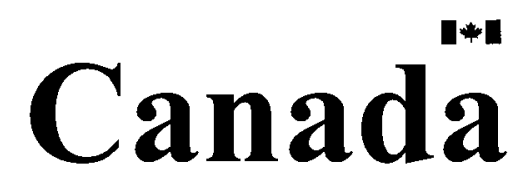




\section{ABSTRACT}

The thesis describes the development and implementation of the basic concept of a Virtual Dressing Room (VDR) simulation, which is a system to allow customers to use their own 3D models to 'try on' outfits and see how they look before they buy them. The primary objective of the project is to design a multilayer perceptron neural network for the virtual dressing room.

This project was divided into three main tasks: (1) theory of creation of the 3D model, (2) design and implementation of a multilayer perceptron neural network, and (3) implementation of the VDR system user interface. This thesis project is mainly focused on designing the neural network. The multilayer perceptron neural network is designed to retrieve a particular preference of garments for a specific customer depending on his or her age, gender and the ethnicity. Thus, the customer does not have to go through the entire database of the garments to select one piece of clothes based on his or her interest. The database will contain all sorts of clothes: wedding dresses for females and suits for males, sports wears for both genders, night gowns, and they might also have unique dresses that are of interest to specific cultures such as Sarees for South Asians, etc. For example, a customer from Africa will not waste his or her time by going through the entire database to choose one for his or her preference. The multilayer perceptron neural network will choose the dresses for their specific interest. The neural network is trained for each ethnic group, but if someone comes with multiple ethnic backgrounds such as African American, the neural network will generalize the preference of the garments for them too. 


\section{ACKNOWLEDGEMENT}

I would like to express my sincere thanks to my supervisors Professors Peter Liu and Brahim Chebbi for their support, patience and well-anchored guidance throughout the process of this thesis.

I would also like to express my very special thanks to my parents, and to my brother and sister. Without their support and encouragement, this work would not have been possible. 


\section{TABLE OF CONTENTS}

\section{ABSTRACT ….........................................................................................................II}

LIST OF TABLES

VII

LIST OF FIGURES

IXX

\section{CHAPTER 1 - INTRODUCTION .......................................................1}

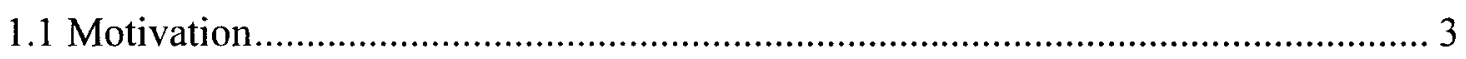

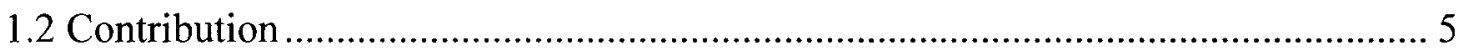

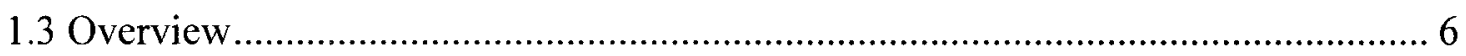

CHAPTER 2 - BACKGROUND AND ANALYSIS....................7

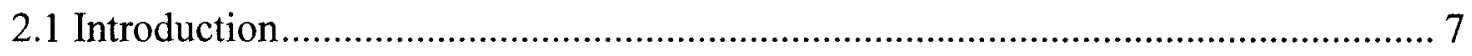

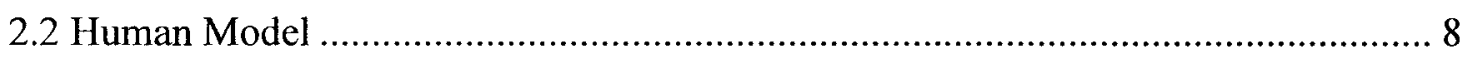

2.2.1 3D Model Reconstruction Using Humanoid Model ...............................................

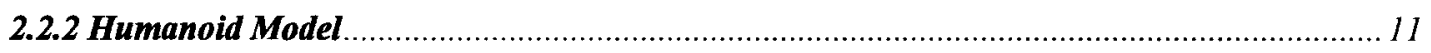

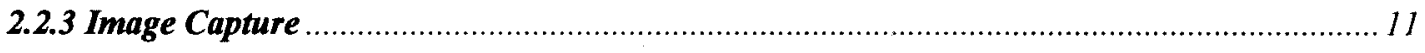

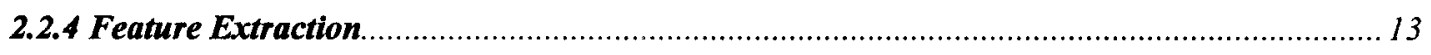

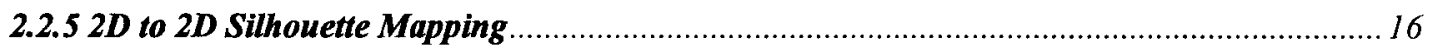

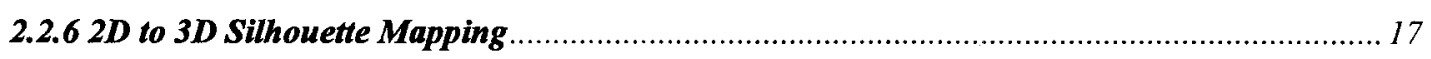

2.3 Acquiring Images from the Remote Nodes ....................................................... 18

2.3.1 Maximized Speedup through Performance Prediction ................................................. 19 
2.3.3 Improving the Performance with Speculation............................................................ 22

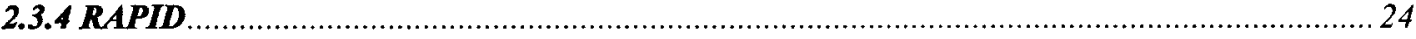

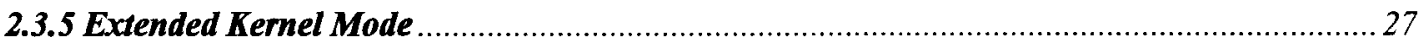

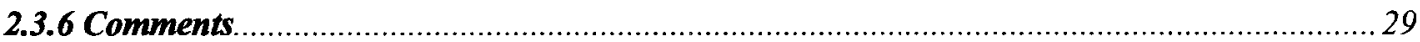

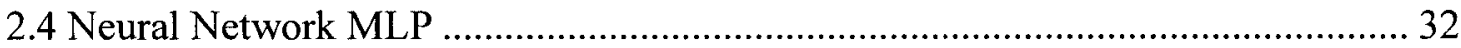

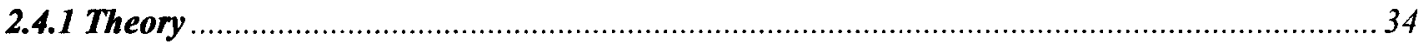

2.4.2 Architecture and Design of Lyapunov Stability-Based Adaptive Back-Propagation (LABP) 35

2.4.3 Architecture and Design of Recursive Least Squares Algorithm (RLS) ........................... 38

\section{CHAPTER 3 - VIRTUAL DRESSING ROOM SYSTEM .....41}

3.1 Stations and VDR System User Interface .................................................. 41

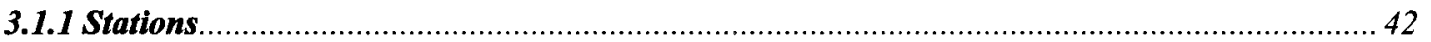

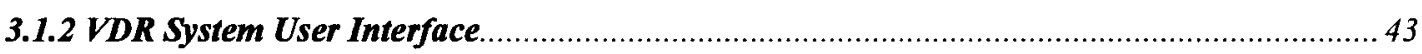

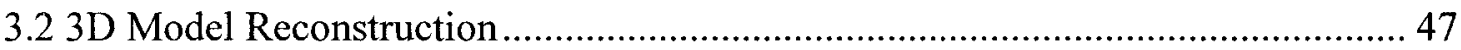

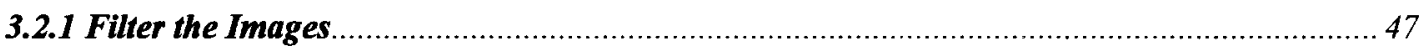

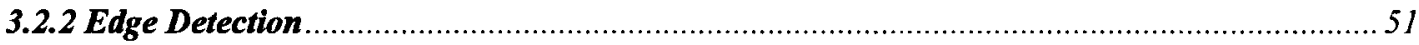

3.2.3 Implementation of 3D Model Reconstruction ......................................................... 54

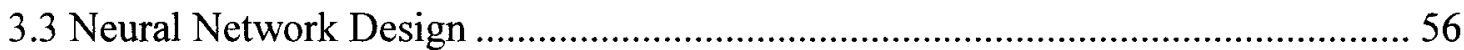

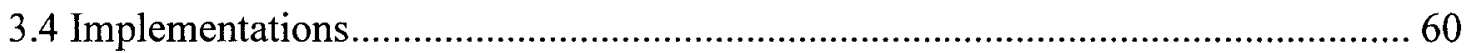

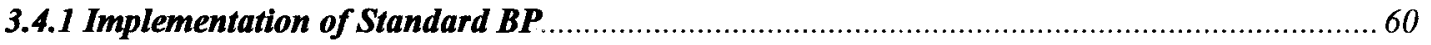

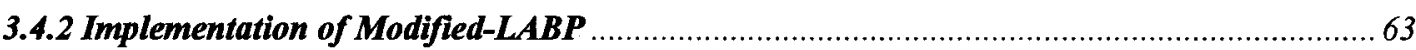

CHAPTER 4 - RESULTS AND DISCUSSION.......................65

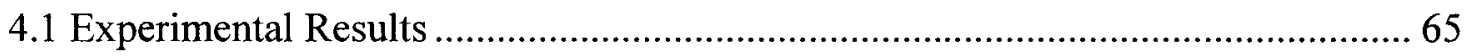

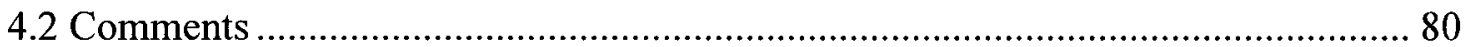


CHAPTER 5 - CONCLUSION AND FUTURE WORK .........82

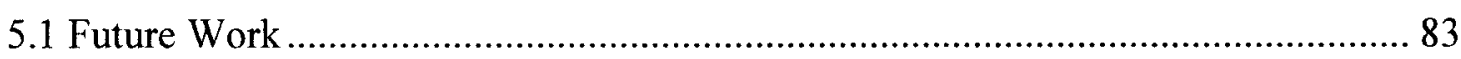

REFERENCE ....................................................................................................88

APPENDIX A - THE RESULTED IMAGES .............................88 


\section{LIST OF TABLES}

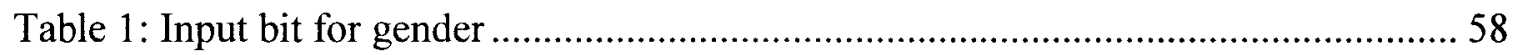

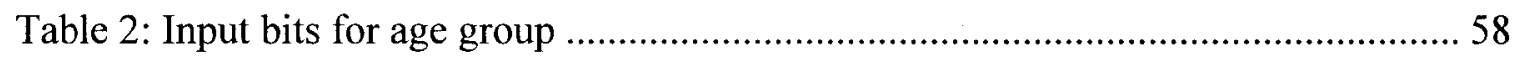

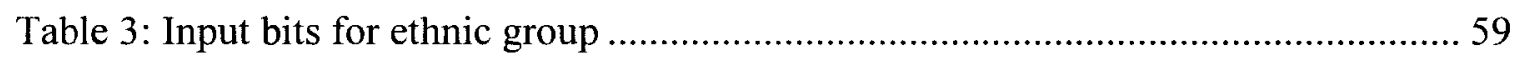

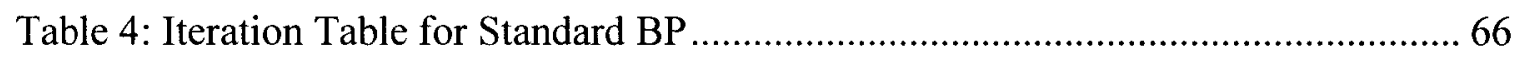

Table 5: Inputs and Outputs from Standard BP Algorithm ............................................ 66

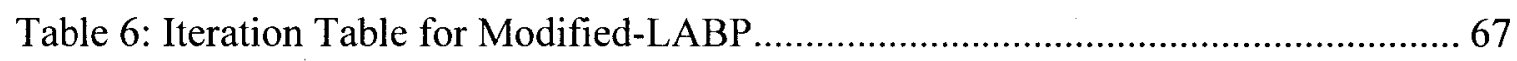

Table 7: Inputs and Outputs from Modified-LABP Algorithm ....................................... 68

Table 8: A set of Sample Inputs and Desired Outputs................................................. 71

Table 9: A set of Sample Inputs and Outputs from Standard BP Algorithm for VDR

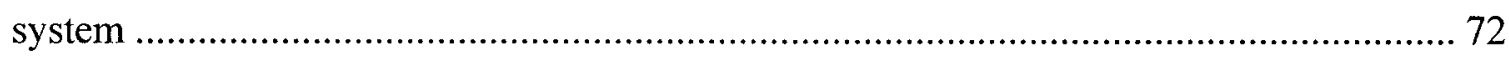

Table 10: A set of Sample Inputs and Outputs from Modified-LABP Algorithm for VDR system

Table 11: A set of Sample Inputs and Outputs from Modified-LABP Algorithm for VDR

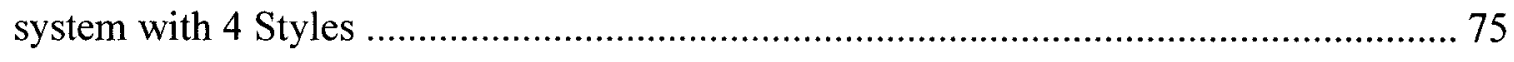

Table 12: A set of Sample Inputs and Outputs from Modified-LABP Algorithm for VDR

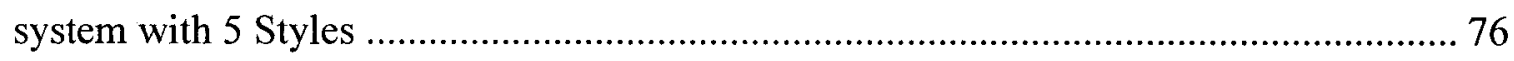

Table 13: A set of Sample Inputs and Desired Outputs for 7 Styles ................................. 77

Table 14: A set of Sample Inputs and Outputs from Modified-LABP Algorithm for VDR

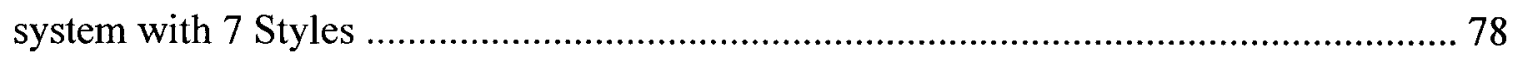

Table 15: A set of Sample Inputs and Outputs from Modified-LABP Algorithm for VDR

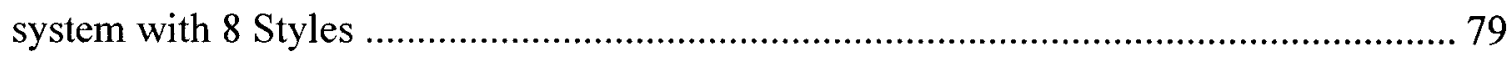


Table 16: A Sample Generalization Result. 


\section{LIST OF FIGURES}

Figure 1: Overview of 3D Model Reconstruction [1] ................................................ 10

Figure 2: Image with Extracted Features [1] ................................................................ 13

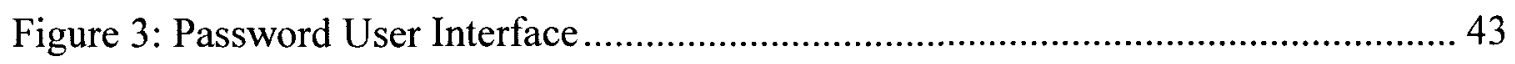

Figure 4: Main VDR System User Interface ............................................................... 44

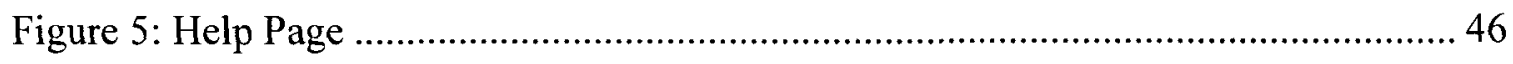

Figure 6: Original Image and Filtered Image ……………......................................... 50

Figure 7: Silhouette Images of Front and Side View.................................................... 53

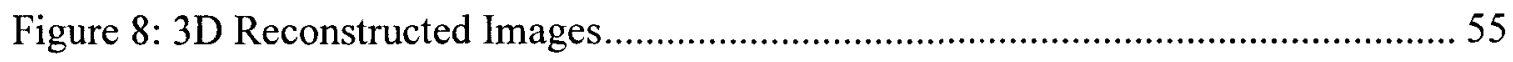

Figure 9: Architecture of the Neural Network ……………........................................ 57

Figure 10: Desired and Actual outputs with added Noise level. ....................................... 74

Figure 11: Original and Gaussian and Salt and Pepper Noisy Images ............................. 88

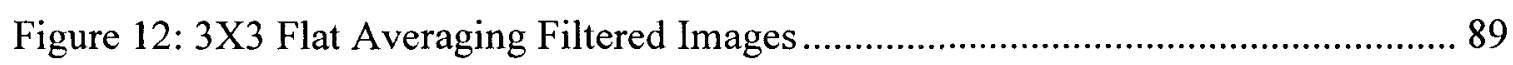

Figure 13: 7X7 Flat Averaging Filtered Images .............................................................. 89

Figure 14: 3X3 Directional Averaging Filtered Images ………....................................... 90

Figure 15: 7X7 Directional Averaging Filtered Images .................................................. 90

Figure 16: 7X7 Gaussian Filtered Images .................................................................... 91

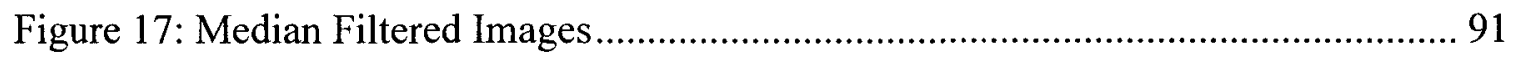

Figure 18: Canny Edge Detector on Original and Gaussian Filtered Gaussian and Salt and

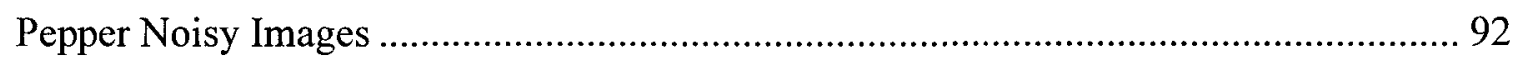

Figure 19: Canny Edge Detector on Median Filtered Gaussian and Salt and Pepper Noisy

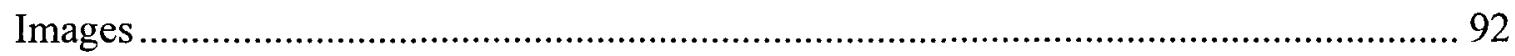


Figure 20: Log Edge Detector on Original and Gaussian Filtered Gaussian and Salt and Pepper Noisy Images

Figure 21: Log Edge Detector on Median Filtered Gaussian and Salt and Pepper Noisy Images

Figure 22: Prewitt Edge Detector on Original and Gaussian Filtered Gaussian and Salt and Pepper Noisy Images

Figure 23: Prewitt Edge Detector on Median Filtered Gaussian and Salt and Pepper Noisy Images 94

Figure 24: Sobel Edge Detector on Original and Gaussian Filtered Gaussian and Salt and Pepper Noisy Images 95

Figure 25: Sobel Edge Detector on Median Filtered Gaussian and Salt and Pepper Noisy Images 95 


\section{CHAPTER 1 - INTRODUCTION}

This thesis project proposes a Virtual Dressing Room (VDR) simulation to allow clothing store customers to use their own 3D model to try the suitability of outfits before they buy them. The purpose of this simulation is to improve the clothing stores' customer friendliness. In clothing stores, the customers have to try on the garments every time to select the one that they want. This will not allow the customer to try more than perhaps 10 or 15 outfits due to the time limits of patience or boredom. Moreover, the customer can try only the materials that are available to them in the store they are currently in. In order to avoid these two circumstances and to improve sales by allowing customers to try more materials in a shorter time period, this project proposes a Virtual Dressing Room (VDR) system. This will allow customers to try clothing on a computer 
without physically wearing them and also to try on the clothes that are offered in other branches of the store without going there.

There will no longer be a need for individual changing rooms. A hall with a few stations will fulfill the purpose of the existing changing rooms. There will be two kinds of stations: one capable of taking photos to create $3 \mathrm{D}$ images in the computer and the other with a computer to try the clothes. Photo stations will each have 4 fixed cameras and a place where the customer is required to stand when the photos are taken. Using these photos, a 3D image of the customer will be reconstructed. The $3 \mathrm{D}$ image can be accessed by the user at the computer station using a password, which will be given to the customer at the photo station. There are many methods to reconstruct $3 \mathrm{D}$ images. In this report, a method is discussed and a modified version of it is implemented for this project.

After the reconstruction of the 3-D model, the user can select garments from the database to try on the model. These images of the garments can be anywhere in the stores' computer network. Distributed shared memory (DSM) is used to acquire these images from remote nodes. Many methods are used in implementing DSM. In this project, the analysis of the five most popular methods has been used to draw a conclusion of the most efficient method.

After having the full 3-D human body model and images of garments, there is a need to display specific garments for a specific customer instead of wasting their time by requiring the customer to go through the entire database. For example, the preferences of the style of a teenaged customer are different from those of a middle-aged customer. In 
addition to age differences, there will be gender, ethnic, shape and size differences. To choose the specific garments for a specific customer, Multilayer Perceptron (MLP) neural network is used. In this project, the neural network will be trained with age, gender and ethnicity as inputs and preference of styles of the garments as outputs. Three methods are discussed in this project: standard back propagation (BP), Lyapunov stability-based adaptive back-propagation (LABP), and recursive least squares (RLS). Afterwards, the standard BP and a new version of LABP are implemented to test and prove that the Modified-LABP gives a better performance for this project.

\subsection{Motivation}

One of the difficult and time consuming aspects of buying a garment is fitting the garment on one individual and seeing the look of the garment on him or her. Even if, a customer likes them, he or she cannot try on all the garments physically. To pick one, they have to choose some garments at first sight and then try on the few they picked. What if the one garment that they ignored would be good on them? They will never know that because they did not try that one.

Another difficult part is whether a particular garment is available in store or not. If it is not in the store, a customer might have to travel to another branch or wait until it is brought in from the warehouse. This is another problem which is time consuming and troublesome. 
These problems can be solved with a virtual dressing room (VDR) system. The customer does not have to try on all the garments physically to see the fitting. However, they can try all the garments in a virtual environment, if they want, because mouse clicking is not going to take much of their time. Moreover, the customer does not have to travel from one branch to another to try out a garment. They can see all the garments that can be purchased from all the branches of the store by viewing through the VDR system.

This project also helps the clothing store managements by improving sale. If a customer cannot find clothes in one store, he or she might not go to another branch of the same store. They might go to another store located in the neighbourhood. The VDR system will prevent these inconveniences. Furthermore, an easy way to see the fitting of the garment will attract more customers to the store. Therefore, this will improve sales in clothing stores. Also, stores would not need large showrooms to display their garments. As the customers can see the garments on the computer, there would be no need for more customer service personnel to help customers to locate garments or to put the garments back on the shelf from the fitting room. This would reduce stores' operating expenditure. The time saving and conveniences are the main reasons for my motivation to propose the VDR system.

The motivation for the neural network, which is the main contribution for this VDR system, is to give an extra feature to the VDR system. In the clothing stores there will be many kinds of outfits: wedding dresses for women and suits for men, night gowns, sports clothes, casual garments, party dresses, and so on. When a customer wants 
to select a casual dress, he or she has to go through the entire database to select one dress. By inputting personal information such as age, gender and ethnicity of the customer through the neural network, the customer's preference of the style of garments will be obtained. As such, customers would not have to go through the entire database to select their clothes.

\subsection{Contribution}

Designing of a neural network to obtain the preference of customers based on their gender, age and ethnicity is my contribution toward VDR system. The neural network will get age, gender, and the ethnic background from the customer. The neural network will be trained with many customers' preferences. From the trained network, the customer will be shown the clothes that suit his or her taste.

A survey is done on DSM systems to choose a better DSM to get the garments' images from remote nodes. The remote access is important because each branch of the clothing store will have their separate servers with the garments that are available in that particular branch. If a customer is not interested in any of the clothing in that particular branch, the VDR system will access the database form other branches of the store. For this process, a better DSM is needed for remote access. 


\subsection{Overview}

In the following sections, a detailed analysis of user interface of VDR system, 3D human model reconstruction, distributed shared memory (DSM), and Multilayer Perceptron (MLP) Neural network are presented with implementations and results of user interface of VDR system, 3D human model reconstruction and MLP Neural network. Subsequently, the report presents a short discussion of future work followed with the conclusion. 


\section{CHAPTER 2 - BACKGROUND AND ANALYSIS}

\subsection{Introduction}

A survey is done on 3D modeling, distributed memory system (DSM) and neural network. The 3D modeling is to reconstruct a $3 \mathrm{D}$ model of the customer and garments so when a customer picks a dress from the database, it will be tried on his or her 3D model.

The DSM is to get all the garments from all servers from other branches of the stores. If there is only one server, there is a danger of loosing the entire database of garments if there is a problem with a server. Further, overload of system access can be generated if many customers form many stores try to access the server at the same time. 
Also, there will be customers who will be happy with the clothes from one store, so no need for remote access at that time. Thus, there is a need for server in each store. If the need of remote access is aroused, the DSM can be used. For this purpose, there is a survey done on DSM to choose the best DSM for this particular system. Last but not least, the survey is done on neural networks to choose the better neural network for this VDR system.

\subsection{Human Model}

A 3D human model is required for Virtual Dressing Room (VDR) system to try garments without the person actually wearing it. There are many methods to reconstruct a 3D model image from photos. Two papers by [2] Cipolla and Giblin, and [3] Zhao and Mohr proposed 3D image surface reconstruction using apparent contours. 3D image quadric surface reconstruction is proposed by Cross and Zisserman [4]. Conic surface reconstruction for photo-grammetry techniques proposed Slama [5]. Sullivan and Ponce proposed silhouette with B-splines based reconstruction methods [6]. Two papers [7], [8] proposed the method, which deform a mesh model to fit the actual image of the person. Furthermore, the paper by Chang and Gerhard proposed free form surface reconstruction [9]. [1] proposed the reconstruction of $3 \mathrm{D}$ image using a humanoid model and four orthogonal view images. The details of this method are discussed in the following section because the modification of this method is used for this VDR system implementation. 


\subsubsection{D Model Reconstruction Using Humanoid Model}

To generate a 3-D human model of a customer, a generic humanoid 3D model is used [1]. From this generic model four orthogonal views (front, back and both sides) images are produced. These are already saved in the database. When a customer stands on the landmark in front of the camera four orthogonal view photos will be taken. These images, called data images, will undergo filtering, edge detection and creation of silhouette images. After the creation of silhouette images on both generic humanoid model and data orthogonal view images, a set of key feature points will be found on both sets of images. The feature points on the model and data images are aligned to match both sets of images. From these silhouette images, with the feature points, 2D-to-2D linear affine mapping creates sets of dense images [1]. These dense $2 \mathrm{D}$ images are then used to define the deformation for the $3 \mathrm{D}$ model [1]. Using this deformation on the humanoid 3D model, the 3D model of an actual human body will be reconstructed. The details of each step is discussed in the following sub-sections. Figure 1 shows an overview of the algorithm: $1 \mathrm{a}$ is the humanoid model, $1 \mathrm{~b}$ is orthogonal images of humanoid model, $1 \mathrm{c}$ is the orthogonal views of a person, $1 \mathrm{~d}$ is the silhouette images of the humanoid model with features, $1 \mathrm{e}$ is the silhouette images of the person with features, If is the dense images and $1 \mathrm{~g}$ is the $3 \mathrm{D}$ model [1]. 


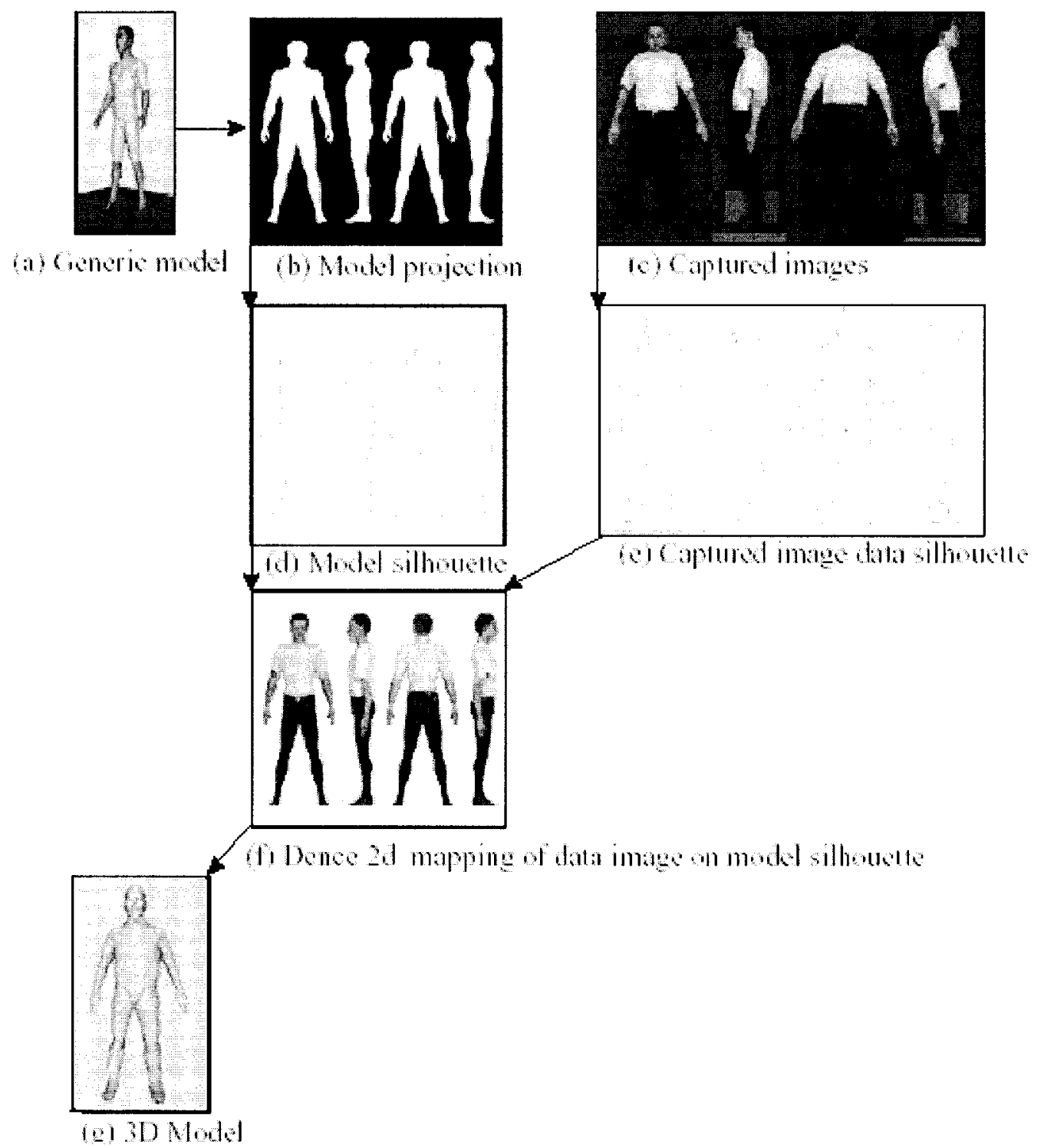

Figure 1: Overview of 3D Model Reconstruction [1]

In this section, reconstruction of 3D model of a human body will be discussed in detail. The model is built using Matlab. The following sections will go through the process in detail. 


\subsubsection{Humanoid Model}

Any public available humanoid model can be used for this algorithm. This humanoid model is a $3 \mathrm{D}$ polygonal mesh segment [1].

$$
\vec{u}_{j}=\{u, v\}_{j=1}^{N_{p}}
$$

Here $U_{j}^{N p}$ is to represent the polygonal mesh with a list of $N_{p} 3 D$ vertices.

\subsubsection{Image Capture}

Using the cameras, all the four orthogonal view images are taken. This will give a set of four data images of a customer.

$$
I_{i}^{D}, i=1,2,3,4
$$


The homogeneous coordinate of the camera $3 \mathrm{D}$ to $2 \mathrm{D}$ projection is

$$
\vec{u}_{i}^{\prime}=P_{i} \vec{x}^{\prime}=M E_{i} \vec{x}^{\prime}
$$

where

$\vec{u}^{\prime}=(u, v, w)-3 \mathrm{D}$ point

$\vec{u}=(u / w, v / w)-2 \mathrm{D}$ point in the camera image plane

$\vec{x}^{\prime}=(x, y, z, 1)-3 \mathrm{D}$ point

$\vec{x}=(x, y, z)-$ world homogenous coordinate

$\mathrm{P}$ is $4 \times 3$ projection matrix, which is changed into $3 \times 4$ camera calibration matrix $M . E_{i}$ is the extrinsic parameter of view transformation.

$$
\begin{gathered}
M=\left[\begin{array}{cccc}
f_{u} & 0 & o_{u} & 0 \\
0 & f_{v} & o_{v} & 0 \\
0 & 0 & 1 & 0
\end{array}\right] \\
E_{i}=\left[\begin{array}{cc}
R_{i} & \vec{t}_{i} \\
\overrightarrow{0}^{T} & 1
\end{array}\right]
\end{gathered}
$$

where $R_{i}$ is $3 \times 3$ rotation matrix and $t_{i}$ is $3 \times 1$ translation vector. 0 is $3 \times 1$ vector of zeros. $f_{u}$ and $f_{v}$ are camera calibration parameters (focal lengths) and $o_{u}$ and $o_{v}$ are image origin.

$f_{u}=$ vertical pixels $x$ distance to camera vertical height

$f_{v}=$ horizontal pixels $x$ distance to camera

horizontal height 


\subsubsection{Feature Extraction}

The key features need to be extracted in order to establish the correct correspondence between humanoid orthogonal view images and data images. Figure 2 shows the front orthogonal view image with features [1].

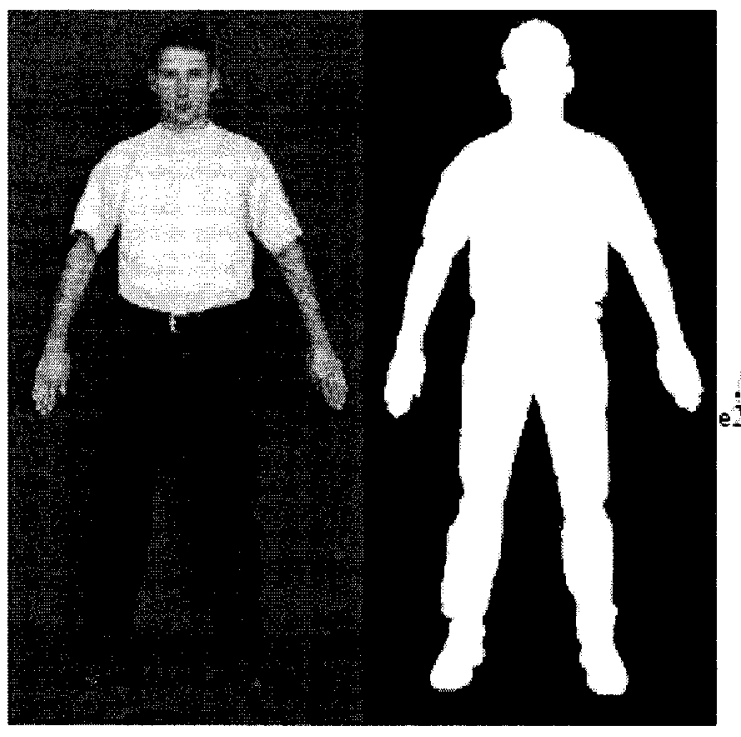

(a) Image

b) Sithouete

(c) Extrema

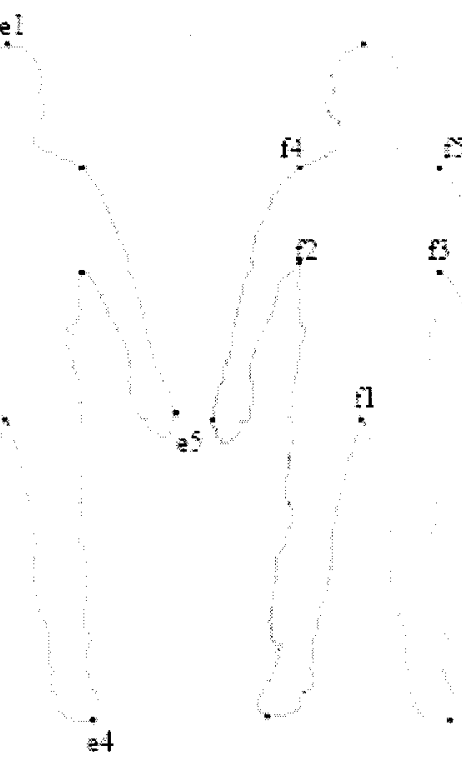

(d) Features

Figure 2: Image with Extracted Features [1]

An algorithm to find feature points is given as follows [1]. First, it explains about finding the extrema points $\mathrm{u}_{\mathrm{el-e}}$ as shown in figure 2c. To find the extrema points on the top of the head, the following formula is used,

$$
\begin{gathered}
u_{e 1}=\min \left(\left\{u_{j}\right\}_{j=1}^{N_{p}}\right) \quad \text { and } \\
v_{e 1}=v_{j}
\end{gathered}
$$

The minimum is used here because the top of the head is the contour point with minimum vertical coordinate $u$. 
To calculate the extrema points on the left arm, $\mathrm{u}_{\mathrm{e} 2}$ and right arm, $\mathrm{u}_{\mathrm{e} 5}$, the following formula is used,

$$
\begin{gathered}
v_{e 2}=\min \left(\left\{v_{j}\right\}_{j=1}^{N_{p}}\right) \quad \text { and } \\
u_{e 2}=u_{j} \\
v_{e 5}=\max \left(\left\{v_{j}\right\}_{j=1}^{N_{p}}\right) \quad \text { and } \\
u_{e 5}=u_{j}
\end{gathered}
$$

Because the arms take as the minimum and maximum on horizontal coordinates on the contour points, here the minimum and maximum of horizontal coordinates, $\mathrm{v}$, are used.

To evaluate the centroid of the silhouette image, the following equation is used

$$
\vec{u}_{c}=\frac{1}{N_{p}} \sum_{j=1}^{N_{p}} \vec{u}(i)
$$

To calculate the extrema points on the left feet, $u_{e 3}$ and right feet, $u_{e 4}$, the maximum vertical coordinate of the contour on either side of the centroid, $\mathrm{v}_{\mathrm{c}}$, is used.

$$
\begin{gathered}
u_{e 3}=\max \left(\left\{u_{j}\right\}_{j=1}^{N_{p}}\right) \quad \text { and } \\
v_{e 3}=v_{j} \leq v_{c} \\
u_{e 4}=\max \left(\left\{u_{j}\right\}_{j=1}^{N_{p}}\right) \quad \text { and } \\
v_{e 4}=v_{j} \geq v_{c}
\end{gathered}
$$


After finding the extrema points, the algorithm went on and explained how to extract the feature points shown in figure $2 d$. The feature points for crotch $u_{f 1}$, left arm $\mathrm{u}_{\mathrm{f} 2}$, and right arm $\mathrm{u}_{\mathrm{f} 3}$ are evaluated with vertical coordinates, $u$.

Crotch

$$
\begin{gathered}
u_{f 1}=\min \left(\left\{u_{j}\right\}_{j=e 3}^{e 4}\right) \quad \text { and } \\
v_{f 1}=v_{j}
\end{gathered}
$$

Left arm

$$
\begin{gathered}
u_{f 2}=\min \left(\left\{u_{j}\right\}_{j=e 2}^{e 3}\right) \quad \text { and } \\
v_{f 2}=v_{j}
\end{gathered}
$$

Right arm

$$
\begin{gathered}
u_{f 3}=\min \left(\left\{u_{j}\right\}_{j=e 4}^{e 5}\right) \text { and } \\
v_{f 3}=v_{j}
\end{gathered}
$$

The feature points for left shoulder $\mathrm{u}_{\mathrm{f} 4}$, and right shoulder $\mathrm{u}_{\mathrm{f}}$ are evaluated with horizontal coordinates, $v$.

Left shoulder

$$
\begin{gathered}
u_{f 4}=\min \left(\left\{u_{j}\right\}_{j=e 1}^{e 2}\right) \text { and } \\
v_{f 4}=v_{f 2}
\end{gathered}
$$

Right shoulder

$$
\begin{gathered}
u_{f 5}=\min \left(\left\{u_{j}\right\}_{j=e s}^{l e l}\right) \text { and } \\
v_{f 5}=v_{f 3}
\end{gathered}
$$




\subsubsection{D to 2D Silhouette Mapping}

After the feature point extraction, using these points, a dense image is created. As it is mentioned in the feature points extraction the body parts can be separated into seven parts: head, shoulder, left arm, right arm, torso, left feet and right feet. By a 2D linear mapping, a body part can be created using the humanoid model and the data image.

$$
\vec{u}^{\prime D}=S \vec{u}^{M}
$$

Equation 27 coordinates a particular body part in the mapping, which is between the corresponding silhouette images of humanoid and data.

$$
S=\left[\begin{array}{ccc}
s_{u} & s_{u v} & t_{u} \\
s_{v u} & s_{v} & t_{v} \\
0 & 0 & 1
\end{array}\right]
$$

where $\mathrm{s}$ represents rotation, shear and scale; and $\mathrm{t}$ represents the translation. $\mathrm{s}_{\mathrm{v}}$ represents the horizontal scaling factor and $s_{u}$ represents the vertical scaling factor. Because of the principal axis of the body parts are aligned, $\mathrm{s}_{\mathrm{uv}}$ and $\mathrm{s}_{\mathrm{vu}}$ are zero.

$$
\begin{gathered}
s_{u}=\frac{u_{\max }^{D}-u_{\min }^{D}}{u_{\max }^{M}-u_{\min }^{M}} \\
t_{u}=-s_{u} u_{\min }^{M}+u_{\min }^{D}
\end{gathered}
$$




$$
\begin{gathered}
s_{v}(u)=\frac{v_{\max }^{D}(u)-v_{\min }^{D}(u)}{v_{\text {max }}^{M}(u)-v_{\min }^{M}(u)} \\
t_{v}(u)=-s_{v}(u) v_{\min }^{M}(u)+v_{\min }^{D}(u)
\end{gathered}
$$

\subsubsection{D to 3D Silhouette Mapping}

After $2 \mathrm{D}$ to $2 \mathrm{D}$ mapping, the next step is $2 \mathrm{D}$ to $3 \mathrm{D}$ mapping. For this affine transform from orthogonal $2 \mathrm{D}$ to $3 \mathrm{D}$ model, the $3 \mathrm{D}$ displacement is required. For the displacement component, first inverse projections must be calculated from the following formula:

$$
\begin{aligned}
\vec{x}^{\prime D}=\vec{x}^{\prime M}+\Delta \bar{x}_{i} & =\lambda_{i}\left(\vec{x}^{D}\right) P_{i}^{-1}\left(\vec{u}_{i}^{M}+\delta \bar{u}_{i}^{\prime}\right) \\
& =\lambda_{i}\left(\bar{x}^{D}\right) E_{i}^{-1} M^{-1}\left(\bar{u}_{i}^{\prime M}+\Delta \bar{u}_{i}^{\prime}\right)
\end{aligned}
$$

Where $\lambda_{\mathrm{j}}$ represents the scaling factor.

$$
\begin{aligned}
& M=\left[\begin{array}{ccc}
\frac{1}{f_{u}} & 0 & \frac{-o_{u}}{f_{u}} \\
0 & \frac{1}{f_{v}} & \frac{-o_{v}}{f_{v}} \\
0 & 0 & 1 \\
0 & 0 & 0
\end{array}\right] \\
& E_{i}=\left[\begin{array}{cc}
R_{i}^{-1} & -R_{i}^{-1} \bar{t}_{i} \\
\overrightarrow{0}^{T} & 1
\end{array}\right]
\end{aligned}
$$




$$
\vec{x}^{D}=\vec{x}^{M}+\Delta \vec{x}_{i}=\lambda_{i}\left(\vec{x}^{D}\right) R_{i}^{-1} M^{-1}\left(\vec{u}_{i}^{M}+\Delta \vec{u}_{i}\right)-R_{i}^{-1} \vec{t}_{i}
$$

Using the above formula the 3D displacement component can be calculated as follows

$$
\Delta \vec{x}_{i} \approx \lambda_{i}\left(\vec{x}^{M}\right) R_{i}^{-1} M^{-1}\left(\vec{u}_{i}^{M}+\Delta \vec{u}_{i}\right)-R_{i}^{-1} \vec{t}_{i}-\vec{x}^{M}
$$

When the estimated displacement component is put on the vertex on the $3 \mathrm{D}$ humanoid mode it will give affine transform to orthogonal to $3 \mathrm{D}$ image.

\subsection{Acquiring Images from the Remote Nodes}

Parallel processing system is an important task in this project because the garments' images may not be stored in one computer and there is a need to bring a collection of garments from other computers (nodes) by high speed network. Distributed Shared Memory (DSM) is a system, which supports to bring data from remote nodes to the local nodes for processing the single computing. For this project, a better DSM system is needed. A survey is done on it to choose the better DSM system.

There are two kinds of DSM systems: software DSM system and hardware DSM system. The DSM system is implemented using many methods. All these methods are to improve the performance of the system. One of the many challenges dealing with DSM is remote memory access latency. Many methods are implemented to reduce the remote memory access latency to achieve better performance. For this project, five of the many 
methods, which were proposed by many talented authors, are analyzed: maximized speedup through performance prediction [10], hiding latency through bulk transfer [11], improving performance with speculation [12], RAPID [13], and extended kernel mode [14].

\subsubsection{Maximized Speedup through Performance Prediction}

The major interest of implementing a parallel processing system is to improve the

performance. Normally, a lager DSM system gives better speed. However, because of the overhead cost for large size of DSM system, the performance could not be improved or even degraded. Therefore, the best performance can be achieved by determining the appropriate size of the DSM system [10].

In the paper, "Maximizing Speedup through Performance Prediction for Distributed Shared Memory Systems," a performance prediction model is proposed. A DSM system Proteus is implemented. The runtime information was used to predict the performance of any parallel applications, which run on Proteus. Proteus DSM system contains a set of runtime Library to provide globally shared address space among nodes; Ethernet connects the nodes. Proteus also supports dynamic node reconfiguration that allows them to add or remove a new computer anytime; that is Proteus supports node reconfiguration to adjust the system size at runtime. Proteus also supports per-node multithreading, thread migration, synchronization primitives, and load balancing [10]. 
The prediction method was used to collect runtime information. Using this runtime information, the execution time of application was predicted under different system sizes. The following equation was used to predict the time application,

$$
t(n)=\frac{t_{s}}{n} t_{p o}(n)
$$

$t(n)$ is the predicted time of application; $t_{s}$ is the time of application executing in one node; $n$ is number of nodes used for the execution; and $t_{p o}$ is the time induced by parallelizing overhead $[10]$ and this $\mathrm{t}_{\mathrm{po}}$ can be predicted by using following formula,

$$
t_{p o}(n)=t_{\text {sys }}(n)+t_{\text {com }}(n)+t_{\text {wait }}(n)
$$

$t_{c o m}$ is communication overhead; $t_{s y s}$ is system overhead; and $t_{\text {wait }}$ is waiting and load balancing overhead. Using $t(n)$, the predicted time of application, the DSM size is adjusted to appropriate size which gives the best performance [10].

By using this performance prediction mechanism, the appropriate system size, which gives max speedup, is determined. The experiment result shows that the accuracy of the performance prediction mechanism is acceptable [10]. 


\subsubsection{Hiding Latency through Bulk Transfer and Prefetching}

DSM machine achieves high performance by caching of sharing data. This will reduce the remote node usage, so the remote access latency will be reduced. But this works fine and gives good performance only on fine-grain pattern. In coarse-grain pattern, the performance is reduced by cache miss and remote access latency because coarse-grain pattern has small size of cache compare to shared memory in the system. This limitation can be overcome by software prefetching; however, there are some critical problems in this approach. One of the problems is "memory latency widely varies depending on the remote node in which the required data is located" [11].

To overcome these problems and to get good performance, a bulk transfer and prefetching method is proposed in the paper, "Hiding Latency Through Bulk Transfer and Prefetching in Distributed Shared Memory Multiprocessors." First, the remote access latency was overcome by converting remote access into local access. For this, a part of local memory was used as a destination of the bulk transfer. The Adaptive Granularity (AG) was used for bulk transfer. When a node requests data, the remote node will send a bulk of data; but, when false sharing expected, the remote node only sends half of the bulk data, which contains the requested data. If there is invalidation message, it will only affect the half bulk. Therefore, the memory latency problem is solved because the data is in local node. AG also guarantees the consistency of the replication data in local memory [11]. 
After the data is replicated and stored in local memory, software prefetching is used to overcome the local access latency. This software prefetching is developed into uni-processor; so, the frequently coherent traffic problem, which is caused by multiprocessors specific characteristics, is overcome [11].

When this bulk transfer and prefetching method are simulated, the result shows that the memory latency for local and remote access was reduced by $25-40 \%$ [11].

\subsubsection{Improving the Performance with Speculation}

DSM brings data, which is located in remote node to local node for processing. DSM cache data from remote memory to local memory and runs coherent protocol to ensure all replicas of data remains coherent to improve the performance. Because of high overhead of the coherent operation, software DSM performance is degraded [12].

In the paper, "Improving the Performance of Software Distributed Shared Memory with Speculation," Speculation Home-based Release Consistency (SHRC) method is proposed that uses speculation to improve the performance. Speculation is executing an operation before it is actually needed. "Speculation can improve the performance by weakening dependencies in program execution" [12]. Speculation predicts the subordinate processing, which will be needed in the future use, and execute it before the outcome is required. This speculation was used here to predict the remote 
data, which will be required later. Then, a protocol was used to transfer the remote data from the home node to the local node before the data is needed. This mechanism, which uses speculation, is implemented in the home node (remote node) [12].

They modified Home-based Lazy Release Consistency (HLRC) protocol into Speculation Home-based Release Consistency (SHRC), which is responsible for maintaining and distributing the data store on the memory space to other nodes. This protocol implies that it will only send the updated version of the data to other nodes if only they were asked. The extended version of HLRC protocol, SHRC, will speculatively perform certain coherence operation in the data before it is required.

This SHRC maintains a table with patterns of past shared memory access. SHRC protocol will use these patterns to predict the future memory access and transfer them to the local mode. Therefore, local nodes will not try to access remote data from home node. The home node itself sends the remote data to the local node, which may require the data in future. Therefore, there will not be a remote access. It will always be local access.

For the application, which has regular access pattern, this method performs effectively and efficiently. The SHRC improves performance by reducing the remote access latency. 


\subsubsection{RAPID}

There are many methods that were proposed to reduce remote memory access latency. In a large scale DSM, a node, which needs some data from a remote, will broadcast messages to all nodes. The remote node which has the requested data will send a message back to the node which requested the data with requested data "if the data is clean," or forward the request back "if the data is dirty." This transfer undergoes communication with remote nodes to access remote memory. Increase in remote memory access latency is the main problem when the nodes on DSM system increase. The methods, which were proposed to reduce the remote memory access latency, successfully achieved the goal but they were expensive because most of these methods require more bandwidth [13].

In the paper, "RAPID: reconfigurable and scalable all-photonic interconnect for distributed shared memory multiprocessors", there is a method proposed to reduce the remote memory access latency using fibre optical interconnects which provides higher bandwidth and lower latency and lower cost [13].

This RAPID architecture design contains "3-tuples": P, D and G. P is number of processors in a node (an assumption - only one processor is assigned to one node); D is number of nodes in a group; and $G$ is number of groups $(D \geq G$ is another assumption to ensure that every group communicates to every other group). There are two subnetworks: scalable intragroup interconnection (IGI) and scalable intergroup remote 
interconnection (SIRI). All nodes are connected to these sub-networks through the intergroup passive coupler (IGPC). The local and remote communications are separated to ensure efficient implementation. All local interconnects are implemented using waveguide optics and all remote interconnections are implemented using fibre optics because fibre optics can be extended to different length [13].

Each node is identified as $R(d, g)$, where the $d$ is node number and $g$ is the group number. Within the group, all the nodes are connected to multiplexers and demultiplexers. This will help for local and remote communication. The wavelength is reused to design an efficient wavelength assignment strategy. For this strategy, the spatial division multiplexing (DSM) technique is used [13].

Each node is assigned to a wavelength. When node 0 wants to communicate to nodel within the same group (local communication), node 0 will use the wavelength; this wavelength is assigned to nodel to transmit the data. Therefore, each node will receive optical packet at the unique wavelength that is assigned to that node [13].

For remote communication, there are two groups involved: destination and source group. For a specific destination, a source group is assigned to a unique wavelength at which it can transmit the packet to destination group [13].

For message routing in RAPID, there are three possibilities: local message routing, remote message routing and broadcast. If it is a local message routing, the 
source and the destination nodes are in the same group, such as $\mathrm{R}(0, \mathrm{~g} 1)$ and $\mathrm{R}(1, \mathrm{~g} 1)$. The source node uses the pre-assigned wavelength of the destination node to transmit the packet using waveguide optics. If it is a remote communication, the source and destination nodes are not in the same group. The source node will use the specific wavelength, which is assigned to the specific source group, to transmit packet using fibre optics. The node in the destination that receives the packet will forward the packet to the destination node using the wavelength of the destination node. If it broadcasts and the broadcasting is for only the nodes within the group, it will use wavelength 0 to transmit the message. If it is broadcasting for all the groups, the source node uses the specific wavelengths that for the destination group. A node in the destination group gets the message and uses wavelength 0 to broadcast the message to the nodes within the specific destination group [13].

This architecture is much faster and inexpensive and reduces the remote access latency. RAPID maximizing the channel availability for communication and wavelength are reused for local and remote communications. This RAPID architecture provides high bandwidth and highly scalable network with minimized latency. If this achieved by traditional electrical way, it will be much more expensive [13]. 


\subsubsection{Extended Kernel Mode}

Many people are interested in clusters of personal computers. A set of kernel mechanism allows users to control the DSM on this cluster. These parallel applications need processors and memory scalability; but the software DSM depends on the OS virtual memory manager and do not maximize the usage of the local memory for data sharing. Moreover, the DSM uses mechanism to ensure data consistency at the expense of increasing system complexity. To solve these problems, memory is managed globally. For this global memory management, first, OS virtual memory mechanism must be extended. Then a set of module must be provided for basic functionalities with minimum overhead. Later, high-level modules will support the DSM protocols [14].

In this paper, "Distributed Shared Memory in Kernel Mode," a new set of kernel mechanism is proposed to allow users to have full control on the DSM. This new set of kernel mechanism is called MOMEMTO (MOre MEMory Than Others) DSM system. This MOMEMTO extends Linux $2.4 \mathrm{kernel}$ and provides a set of mechanism to support global shared memory. MOMEMTO provides a basic shared mechanism by creating and managing its own virtual memory area (VMA) in kernel. If a user requests memory address, the MOMEMTO creates its own virtual memory address and uses them in process' VMA. Then the process uses this MOMEMTO memory. Each application has virtual memory segment into its address space. Normally, a node will have its own virtual memory segment. In MOMEMTO, all nodes will have their own virtual memory segment. But all the nodes will share them. Therefore, the virtual memory segment is 
managed globally. Individual nodes will allocate the virtual memory segment they own at initialization [14].

MOMEMTO uses three functions: mmt_init, mmt_mmap, and mmt_update. The function mmt_init is to initialize the MOMEMTO. The input parameter is a text file, which contains all the nodes that are going to share the memory. All application must call this function at all the nodes. The function mmt_mmap is to map a VMA that will be shared among the nodes. The input is how much memory is asked by every node. All nodes must call this function. The function $\mathrm{mmt}$-update is called to update virtual memory pages. The latest version will be sent to all other nodes. If the owner node does not have the latest version, the owner's virtual memory page will be updated first; then all the other nodes' virtual memory pages will be updated [14].

MOMEMTO executed in low overhead cost and performed well with small overhead for virtual memory management [14]. 


\subsubsection{Comments}

The first paper [10] analyses the performance prediction mechanism to improve the performance of the parallel processing system. The paper explains how to determine the appropriate size of the DSM, which will give a best speedup. The execution time is predicted using runtime information to adjust the DSM system. In the formula, which was used to predict execution time, there are 3 parameters: $t_{s}, n$, and $t_{p o} . n$ is known parameter and $t_{s}$ and $t_{p o}$ are determined using runtime information. Even though, the experiment gave an acceptable result, $t_{s}$ and $t_{p o}$ must be calculated separately before finding the prediction time to adjust DSM size. It will have extra overhead.

The second paper [11] examines how to reduce the remote access latency. The method that was proposed in this paper to reduce remote access latency was to transfer a bulk of data with requested data to local memory. Therefore, most of the time when the required data will be in the local memory, so the remote memory access latency is reduced. However, if the bulk of data does not contain data which was needed for the specific application, there will be overhead because the size of the transfer data is bigger than the size of the requested data, so higher bandwidth is required. Moreover, remote access is needed because the bulk data does not contain useful data for that specific application. Therefore, this bulk transfer can worsen the problem by adding more overheads with remote memory access latency. But if the data contains useful information for the application, it will give high performance. 
In the third paper [12], a method using speculation is analyzed to improve performance by reducing remote memory access latency. The remote node (home node) has a protocol, SHRC, which predicts the data that is needed in future, using speculation. SHRC protocol maintains a table with past memory pattern. Using this table, SHRC protocol predicts the future memory access and transfer that memory to the local node before it required. If the application has a regular access pattern, this method improves the performance. Otherwise, the performance can be degraded because home node transfer data which is never required by the application. Therefore, with the remote access latency, there will be overhead to transfer not useful data and a higher bandwidth is needed. Therefore, before using this method, determining the pattern of the memory access of the application is required. Thus, this will add another overhead to predict the memory access pattern of the applications.

In the fourth paper [13], a method is proposed to use fibre optical interconnects to transfer data. This will increase the bandwidth and it is inexpensive. Therefore, the RAPID architecture is fast, reduces the remote access latency, and maximizes the channel availability. This method reuses wavelength. It is easy to add or remove a node to a group anytime because the only thing needed is assigning a wavelength to the node if the node is added. If the node is removed nothing will be required to be changed. For communication to the remote node, the wavelength is assign to the group, so each node does not have to care about that. This will be more than one node on a group and work as a one local node. Therefore, if an application is only needed the nodes within the group, there will not be any remote group access needed. 
The fifth paper [14] analyzes a hardware DSM system, MOMEMTO. MOMEMTO is an extended version of Linux 2.4 kernel to support global shared memory management. It provides three basic functions to initialize map and update. There will not be data consistency problem because the virtual memory is shared globally. However, critical section problem will be there. This method also needs more bandwidth when the data is transferred from physical memory to cache.

All those five methods are implemented to improve the DSM system performance in different ways. But, for the purpose of this project, reducing the remote memory access latency is the better method because the bulk of data that contains not-useful data is minimal. 


\subsection{Neural Network MLP}

In the Virtual Dressing Room (VDR) system, if the customer asks for it, the system must find the garments that are most appropriate for the particular customer. For example, if the customer is a teenaged girl, then the materials that fall under categories, female and teenage will be chosen to be displayed. In doing this, it ensures that the customer does not have to go through the entire database to select the desired outfit. The database will contain all sorts of garments such as sports clothing, wedding dresses and night clothing. It will be a waste of time and effort to go through the entire database to select one or two garments. To choose the style of the garments for a specific customer, the Multilayer Perceptron (MLP) Neural network is used. This system will train with customers' age, gender and ethnic group as inputs to choose styles. For example, a teenage boy and a girl may like the same kind of T-shirt. If the VDR system does not use neural network, the database must save the T-shirt in teenage boy section and teenage girl section. This will lead to store one item more than one time and waste of hardware because it is not going to be one or two items. Another example could be a 30-year-old woman and a 50-year-old woman wanting to choose a similar kind of jackets. The neural network will give output which indicates the style of garment that is preferred by age, gender and ethnic group. For example, the teenage girl likes the style group 1 and 3 and teenage boys like the style group 2 and 3 .

Multilayer Perceptron is a network of simple neurons called perceptron. MLP is made up of several layers of neurons. Each layer is fully connected to the next layer. The supervised learning problem of the MLP can be solved with the standard back- 
propagation (BP) algorithm. The standard BP algorithm is based on gradient descent. The weights are modified in the direction that corresponds to the negative gradient of an error measurement. However, there are some drawbacks for standard back-propagation algorithm. One of them is the rate of convergence which is very slow. Second one is that the rate of the convergence is highly dependent on choice of the learning rate, which is pre-specified tolerance. If the learning rate is chosen as a large value, it will lead to rapid learning but weight choice may cause oscillation or become unstable. On the other hand, if the learning rate is chosen as a small value, it will lead to a slow learning. Therefore, for standard back-propagation, the training is dependent on the learning rate, but finding appropriate learning rate is difficult. The third drawback is that the standard backpropagation is prone to become trapped in the nearest local minima and/or fail to find global minimum representing the solution [15]

In this project, two algorithms were analyzed to accelerate the learning algorithm for MLP Networks. One is Lyapunov stability-based adaptive back-propagation (LABP), and the other one is recursive least squares (RLS) algorithm. Standard BP and LABP algorithms are implemented for testing on this project. This will take age, gender and ethnic group as the inputs and preference of the styles as the output. 


\subsubsection{Theory}

Training algorithm learns or updates weight until the mean squared error between the output predicted by the network (or actual output) and the desired output (or target output) is less than a pre-specified small value. The total error can be calculated using the following formula [16]:

$$
E=\frac{1}{N} \sum_{k=1}^{N} E(k)
$$

where,

$$
E(k)=\frac{1}{M} \sum_{i=1}^{M}\left[y_{i}(k)-\hat{y}_{i}(k)\right]^{2}
$$

where $\mathrm{N}$ is number of patterns in the training set, $\mathrm{M}$ is the number if the output nodes, $y_{i}(k)$ is the actual output of the training pattern $\mathrm{k}$ and $\hat{y}_{i}(k)$ is the desired output of the training pattern $\mathrm{k}$. The standard back-propagation updates the weight using the following formula [16]:

$$
w(\text { new })=w(\text { old })-\eta \frac{E(k)}{w}
$$

where $\mathrm{w}$ is the weight and $\eta$ is the learning rate. The main problem here is choosing the proper learning rate, which allows convergence and is required for the stability of the total system. But there is not a specific definition to choose learning rate [16]. 
To overcome this problem, many algorithms were reviewed from the literature. The rest of the report analyses two algorithms to accelerate learning algorithm for MLP. One is Lyapunov stability-based adaptive back-propagation (LABP), and the other one is recursive least squares (RLS).

\subsubsection{Architecture and Design of Lyapunov Stability- Based Adaptive Back-Propagation (LABP)}

Lyapunov stability theory, which is implemented for the standard BP, guarantees the stability of the neural network. This algorithm is called Lyapunov stability- Based Adaptive Back-Propagation. The Lyapunov stability theory adjusts the weight so that the error between the actual output and the target output can converge to zero asymptotically. Moreover, the Lyapunov stability theory ensures that the system will not be stuck on the local minima and will have fast convergence [15].

There are five steps to train this algorithm [15].

Step 1:

The weights $\left(\mathrm{w}_{\mathrm{ij}}\right.$ and $\left.\mathrm{w}_{\mathrm{jl}}\right)$ are randomly initialized. $\mathrm{w}_{\mathrm{ij}}$ represents the connection weight between $\mathrm{i}^{\text {th }}$ input node and $\mathrm{j}^{\text {th }}$ hidden node. Let say, there are $\mathrm{m}$ input nodes and $\mathrm{M}$ hidden nodes. As a result, $\mathrm{i}$ goes from 1 to $\mathrm{m}$ and $\mathrm{j}$ goes from 1 to $\mathrm{M}$. $\mathrm{w}_{\mathrm{jl}}$ represents the connection weight between the $\mathrm{j}^{\text {th }}$ hidden node and $\mathrm{l}^{\text {th }}$ output node. 
Step 2:

The input vector is passed to the nodes in the input layers.

$$
\vec{X}_{i}(k)=\left[x_{1}(k), x_{2}(k), \ldots, x_{m}(k)\right]
$$

where, $\bar{X}_{i}(k)$ represents the input vector of the $\mathrm{k}^{\text {th }}$ training pattern.

Step3:

The output function $\mathrm{q}_{\mathrm{i}}(\mathrm{k})$ is computed.

$$
q_{i}(k)=f_{j}\left(\sum_{i=1}^{m}\left[w_{i j}-x_{i}(k)\right]\right)
$$

where, $f_{j}$ is the activation function,

$$
f_{j}(\bullet)=\frac{1}{1+e^{-\alpha(\bullet)}}
$$

where, $\alpha$ is pre-specified parameter.

After computing the output function, the actual output is computed using the following equation,

$$
y(k)=\sum_{j=1}^{M}\left[w_{j l}-q_{i}(k)\right]
$$

Step 4:

The error between the actual and the target output is computed.

$$
e(k)=y(k)-\hat{y}(k)
$$


Step 5:

The weights are adjusted using the following equations.

$$
w_{j l}(k)=w_{j l}(k-1)+\Delta w_{j l}(k)
$$

where,

$$
\Delta w_{j l}(k)=\frac{1}{q_{i}(k)} \frac{1}{M}\left[\hat{y}(k)-\sum_{j=1}^{M}\left[w_{j l} \bullet q_{i}(k)\right]\right]
$$

and,

$$
w_{i j}(k)=w_{i j}(k-1)+\Delta w_{i j}(k)
$$

where,

$$
\Delta w_{i j}(k)=-w_{i j}(k-1)+\frac{1}{x_{i}(k)} \frac{1}{M} g_{j}(u(k))
$$

where,

$$
u(k)=\frac{1}{w_{\jmath}(k)} \frac{1}{M} \hat{y}(k)
$$

where,

$$
g_{j}(u(k))=1+e^{-\alpha(u(k))}
$$

These weights adjustment leads the error $\mathrm{E}(\mathrm{k})$ to asymptotically converge to zero. 


\subsubsection{Architecture and Design of Recursive Least Squares Algorithm}

(RLS)

The Recursive Least Squares (RLS) algorithm is proposed to speed up the convergence.

A performance measure is introduced in RLS algorithm [17].

$$
Q(k)=\sum_{t=1}^{k} \lambda^{k-t} \sum_{j=1}^{N} E_{j}^{L}(t)^{2}
$$

where,

$$
E_{j}^{L}=\hat{y}_{j}-f\left(\bar{x}^{L}(t)^{T} \cdot \bar{w}_{j}^{L}(k)\right)
$$

where, $\mathrm{N}$ is number of nodes in the output layer, $\lambda$ is a positive constant less than and close to $1, \mathrm{E}_{\mathrm{j}}^{\mathrm{L}}$ represents the error of the $\mathrm{j}^{\text {th }}$ node in the output layer, $\hat{y}_{j}$ is the desired output of the $\mathrm{j}^{\text {th }}$ node, $\vec{x}^{L}$ is vector of inputs for the output layer, and $\vec{w}_{j}^{L}$ is vector of weights of the $\mathrm{j}^{\text {th }}$ node in the output layer.

Partial derivative of $\mathrm{Q}(\mathrm{k})$ is taken with respect to weight and is set to zero to minimize the performance index.

$$
\frac{Q(k)}{\vec{w}_{i}^{n}(k)}=\sum_{t=1}^{k} \lambda^{k-t} \sum_{j=1}^{N} \frac{y_{j}^{L}(t)}{\vec{w}_{i}^{n}(k)} E_{j}^{L}(t)=0
$$


where, $y_{j}^{L}$ is the actual output of the $\mathrm{j}^{\text {th }}$ node in the output layer and $\bar{w}_{i}^{n}$ is the vector of weights of the $\mathrm{i}^{\text {th }}$ node in the $\mathrm{n}^{\text {th }}$ layer.

After some calculation the equation, which is shown above, can be converted to

$$
\sum_{i=1}^{n} \lambda^{k-1} f^{\prime 2}\left(s_{i}^{n}(t)\right)\left[b_{i}^{n}(t)-\bar{x}^{n}(t)^{T} \bar{w}_{i}^{n}(k)\right] \vec{x}^{n}(t)^{T}=0
$$

where,

$$
\begin{gathered}
s_{i}^{n}(t)=\vec{w}_{i}^{n}(k) \vec{x}^{n}(t)^{T} \text { and } \\
b_{i}^{n}(t)=f^{-1}\left(\hat{y}_{j}^{n}\right)
\end{gathered}
$$

The above equation can be presented in the vector form as follows

$$
\vec{r}_{i}^{n}(k)=\bar{R}_{i}^{n}(k) \bar{w}_{i}^{n}(k)
$$

where, vector $\vec{r}_{i}^{n}$ and matrix $\vec{R}_{i}^{n}$ can be calculated by

$$
\begin{aligned}
& \vec{r}_{i}^{n}(k)=\lambda \vec{r}_{i}^{n}(k-1)+f^{\prime 2}\left(s_{i}^{n}(t)\right) \bar{x}^{n}(k) \hat{y}_{i}^{n}(k) \\
& \vec{R}_{i}^{n}(k)=\lambda \vec{R}_{i}^{n}(k-1)+f^{\prime 2}\left(s_{i}^{n}(t)\right) \bar{x}^{n}(k) \bar{x}^{n}(k)^{T}
\end{aligned}
$$

So, the weight can be calculated as follows

$$
\begin{aligned}
\bar{w}_{i}^{n}(k) & =\vec{r}_{i}^{n}(k) \vec{R}_{i}^{n}(k)^{-1} \\
& =\bar{w}_{i}^{n}(k-1)+g_{i}^{n}(k) E_{i}^{n}(k)
\end{aligned}
$$


where,

$$
g_{i}^{n}(k)=\frac{f^{\prime}\left(s_{i}^{n}(k)\right) \bar{R}_{i}^{n}(k-1)^{-1} \bar{x}^{n}(k)}{\lambda+f^{\prime 2}\left(s_{i}^{n}(k)\right) \bar{x}^{n}(k)^{T} \bar{R}_{i}^{n}(k-1)^{-1} \bar{x}^{n}(k)}
$$

The adjustment of the weights leads to speed up the convergence. 


\section{CHAPTER 3 - VIRTUAL DRESSING ROOM SYSTEM}

\subsection{Stations and VDR System User Interface}

There is no need for separate rooms to 'try on' the garments anymore. Instead, there will be a hall with 2 kinds of stations: photo stations to take photos and computer stations to 'try on' the garments. The computers in the computer station have the Virtual Dressing Room (VDR) simulations. This simulation has a VDR system user interface to obtain the inputs from the customers and presents the outputs to the customers. The following sections will discuss the stations and the VDR system user interface in detail. 


\subsubsection{Stations}

The two stations are photo stations and computer stations. The photo stations have 2 cameras, which will be used to acquire orthogonal view (front and side) images of the customer. The cameras are fixed to take the orthogonal images. From those orthogonal images, 3D images will be generated. To access their own $3 \mathrm{D}$ images from the computer station, the customers will be given a password.

The computer station, which has a computer and a user manual for the users' usage, contains a Virtual Dressing Room (VDR) simulation on the computer. Using the VDR simulation, the $3 \mathrm{D}$ images that were reconstructed in the photo station can be used to 'try on' the garments. A database of the images of garments is accessible to the customers. If the customer provides his or her age, gender and ethnicity, the garments with the style that is mostly preferred by that particular age, gender and ethnic group will appear first. If the customer is not satisfied with the chosen garments, they can always view the entire database. 


\subsubsection{VDR System User Interface}

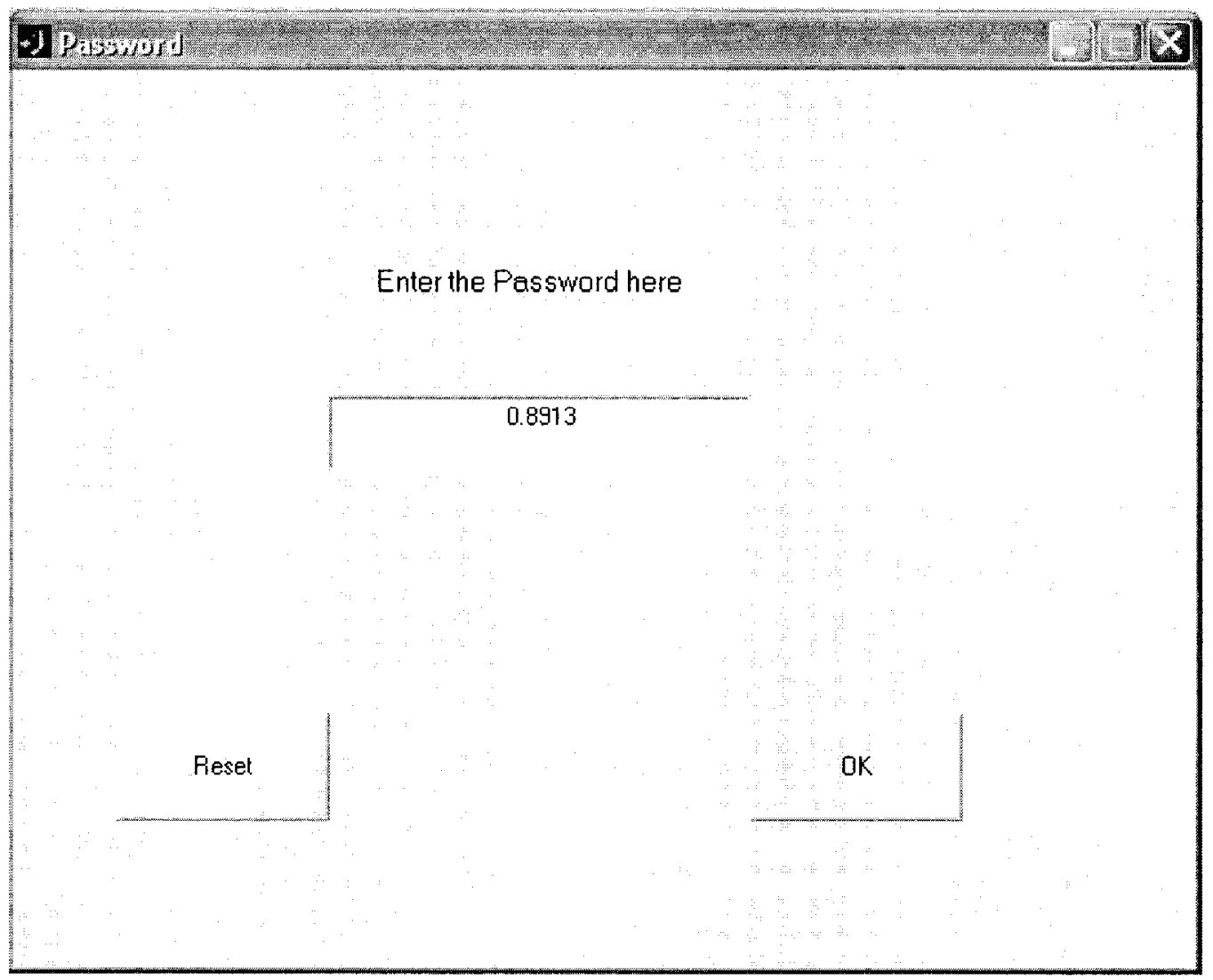

Figure 3: Password User Interface

A user interface is implemented using Matlab to access the Virtual Dressing Room (VDR) system. After the photos are taken, the customer comes to the computer station. The computer shows a window (user interface) as shown in Figure 3 to enter the password, which was given in the photo station. When the customer enters the correct password and presses OK, the main VDR system user interface will open. If the customer fails to give the correct password, an error message will pop up. There is a 'Reset' button to reset and re-enter the password. 


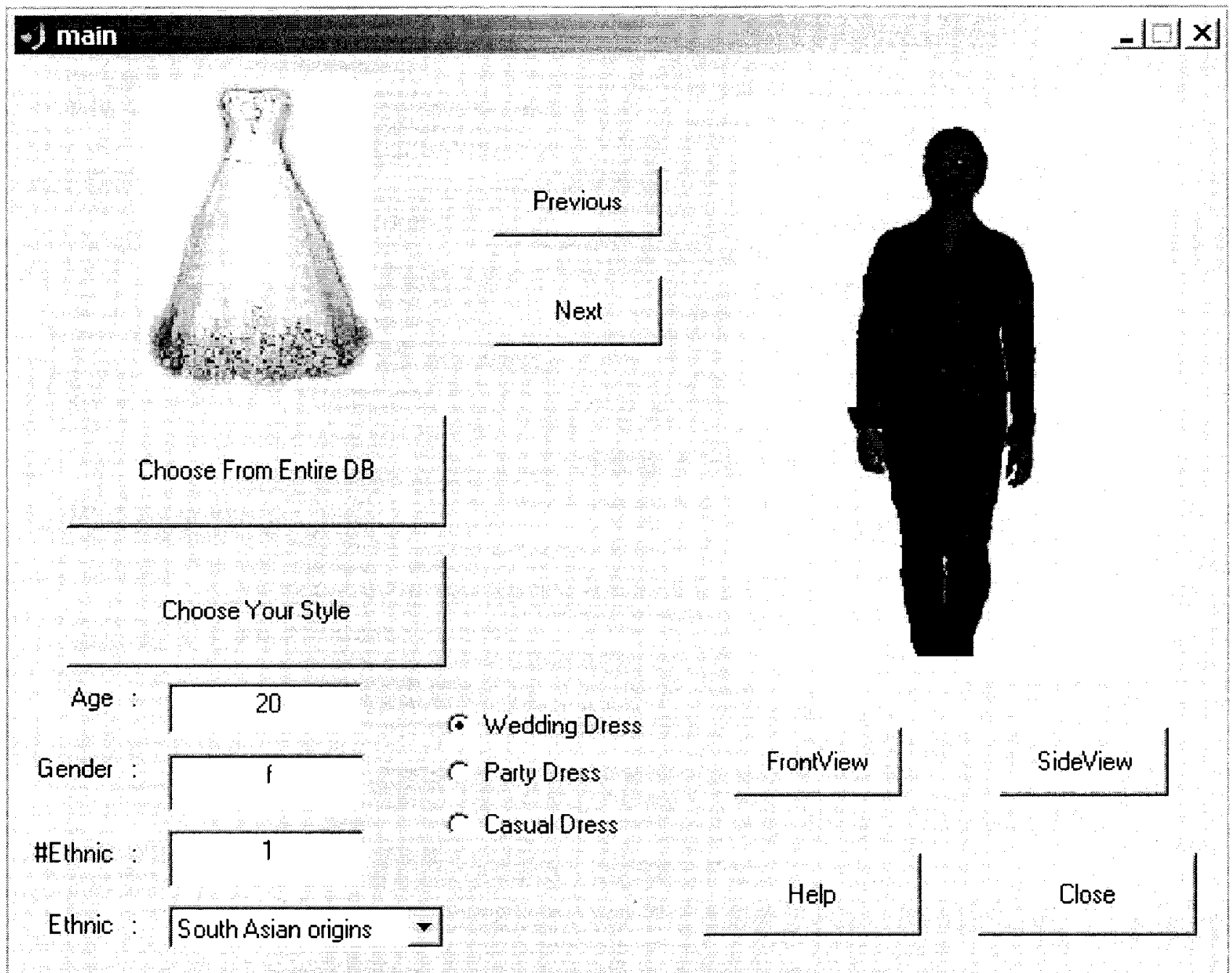

Figure 4: Main VDR System User Interface

When the customer enters the correct password, the main VDR system user interface will be open as illustrated in Figure 4. The 3D image is shown in the top right hand corner. If the customer presses on 'Front View', he or she will be able to see the front view 3D image and he or she can press on 'Side View' to see the side view 3D image. 
On the top left hand corner, the images of the garments from the database are shown. If the customer clicks on 'Choose From Entire DB', all the garments from the database can be seen by pressing 'Previous' and 'Next' buttons. These garments will include wedding dresses, party dresses, and casual dresses, etc.; that is the entire database. There can be sports clothes, gym clothes, and night clothes too, but due to the lack of the real database, the garments are limited to wedding, party, and causal dresses.

Going through the entire database is a waste of time if the customer has come for a particular garment such as a wedding dress. In that case, the user selects the 'Wedding Dress', gives the gender and presses 'Choose Your Style' button to see the wedding dresses from the database. It will exclude party and casual dresses. If the customer is a female, the wedding tuxedos will be excluded as well. The same applies for the party dresses. By selecting the 'Party Dress' and giving the gender, a customer can see the party dresses that are appropriate for them.

If the customer chooses casual dresses, the age, gender and ethnic group are needed. The age must be a number and gender must be typed as 'female', 'male', ' $\mathrm{f}$ ' or ' $\mathrm{m}$ ' and the case is not sensitive. If the customer fails to give proper entry, error messages will pop up. Casual dresses is selected when the customer presses 'Choose Your Style' button, the garments, which are particularly interested by that specific gender, age and ethnic group will be selected from the database and is shown in the top left hand corner. If they do not like the garments that are chosen for them, they can always go to choosing from the entire database. 
Clicking on the 'Help' button will open a window as shown in Figure 5 to help to use main VDR system user interface. This page can be used to give the customer the direction to find the clothes in the store.

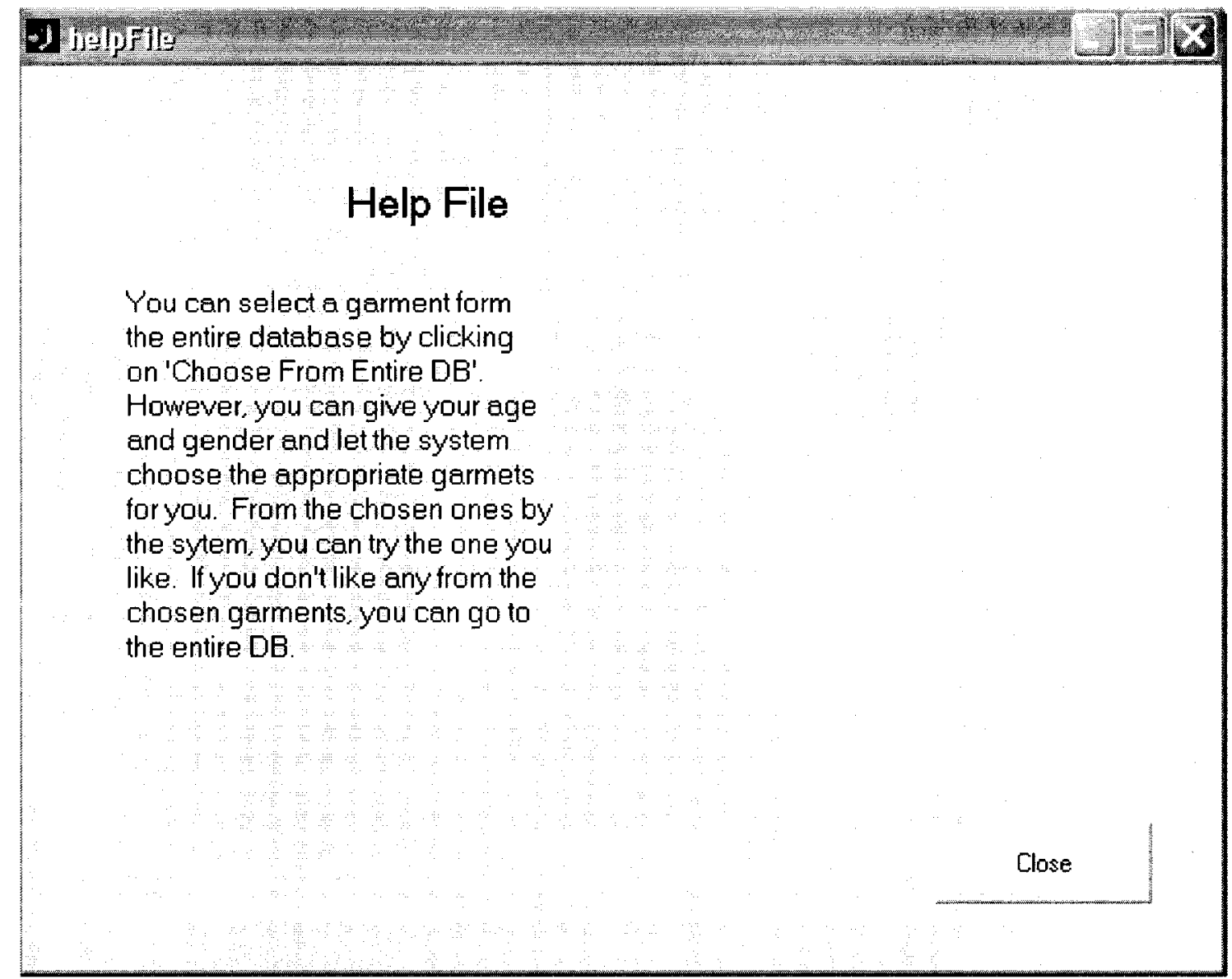

Figure 5: Help Page 


\subsection{D Model Reconstruction}

\subsubsection{Filter the Images}

After capturing the images, they are filtered so it will be easier to detect edges. There are many ways to filter images: flat averaging filter, directional averaging filter, Gaussian filter, and median filter. Noises are added to the images and then filtered to find better silhouette images.

$3 \times 3$ flat averaging filter on original image gives a pure black and white image. The grey color is removed to white. $3 \mathrm{X} 3$ flat averaging filter on Gaussian noisy image removes some of the Gaussian noise, but there is still noise. $3 \mathrm{X} 3$ flat averaging filter on salt and pepper noisy image filtered some of the dark and light spots all over the places, but the salt and pepper noise is not removed entirely. $3 \times 3$ flat averaging filter does not blur the image so much.

$7 X 7$ flat averaging filter on original image gives a pure black and white image. The grey color is removed to white. 7X7 flat averaging filter on Gaussian noisy image removes most of the noisy effect. $7 \mathrm{X} 7$ flat averaging filter on salt and pepper noisy image filtered most of the dark and light spots all over the places, but the salt and pepper noise is not removed completely. $7 \mathrm{X} 7$ flat averaging filter gives better-filtered (noisy removed) images compared to $3 \mathrm{X} 3$ flat averaging filter but it blurs the images. 
$3 \mathrm{X} 3$ directional averaging filter on original image gives the same noise removal performance as $3 \mathrm{X} 3$ flat averaging filter. $3 \mathrm{X} 3$ directional averaging filter on Gaussian noisy image removes noise the same as $3 \times 3$ flat averaging filter. $3 \mathrm{X} 3$ directional averaging filter on salt and pepper noisy image gives almost the same noise removal performance as $3 \mathrm{X} 3$ flat averaging filter, but $3 \times 3$ flat averaging filter is slightly better. $3 \times 3$ directional averaging filter gives better blurring effect than $3 \times 3$ flat averaging filter. That is, the images filtered with $3 \mathrm{X} 3$ flat averaging filter are more blurred than the images filtered with $3 \times 3$ directional averaging filter.

7X7 directional averaging filter on original image gives same noise removal performance as $7 \mathrm{X} 7$ flat averaging filter, but the blurring effect with this one is better than $7 \mathrm{X} 7$ flat averaging filtered images. $7 \mathrm{X} 7$ flat averaging filter on Gaussian noisy image gives same noise removal performance as $7 \mathrm{X} 7$ directional averaging filter, but the blurring effect with this one is better than $7 \times 7$ flat averaging filtered images. $7 \mathrm{X} 7$ flat averaging filter on salt and pepper noisy image gives better noise removal performance as $7 \times 7$ directional averaging filter (this one still contains more dark and light spots), but the blurring effect with this one is better than 7X7 flat averaging filtered images.

$7 \mathrm{X} 7$ Gaussian filter on original image gives same noise removal performance as $7 \mathrm{X} 7$ flat/directional averaging filters, but the blurring effect on this one is better than $7 \mathrm{X} 7$ flat averaging filter, but more blur than $7 \mathrm{X} 7$ directional averaging filtered images. $7 \mathrm{X} 7$ Gaussian filter on Gaussian noisy image gives same noise removal performance as $7 \mathrm{X} 7$ directional averaging filter, but the blurring effect on this one is better than $7 \times 7$ flat 
averaging filter, but more blur than $7 \mathrm{X} 7$ directional averaging filtered images. $7 \mathrm{X} 7$ salt and pepper filter on Gaussian noisy image gives same noise removal performance as 7X7 flat averaging filter, but the blurring effect on this one is better than $7 \mathrm{X} 7$ flat averaging filter, but more blur than $7 \mathrm{X} 7$ directional averaging filtered images.

Median filter on original image does not make any difference. The image is still the same as the original one. It removes some of the Gaussian noise on the Gaussian noisy image, but works really well with salt and pepper noisy image. It removes all the noise and produces an image almost the same as the original.

Median filter works really well with salt and pepper noisy images, and 7X7 directional averaging filter and 7X7 Gaussian filter work better with Gaussian noisy images compared to the other filters. The size of the filter mask is important because it decides the blurring effect and noise removal performance. The larger the filter mask the more blurry the image, but the better the noise removal performance.

The conclusion is reached that the median filter works really well with salt and pepper noisy images, and 7X7 directional averaging filter and 7X7 Gaussian filter work better with Gaussian noisy images compared to the other filters. The size of the filter mask is important because it decides the blurring effect and noise removal performance. The larger the filter mask makes the more blurry the image, but the better the noise removal performance. Therefore, the $7 \times 7$ Gaussian filter is used in this project for filtering the image of the customer. Figure 6 shows the original and the filtered image. 
This image is filtered with $7 \times 7$ Gaussian filter. The other filters' resulted images are in Appendix A.

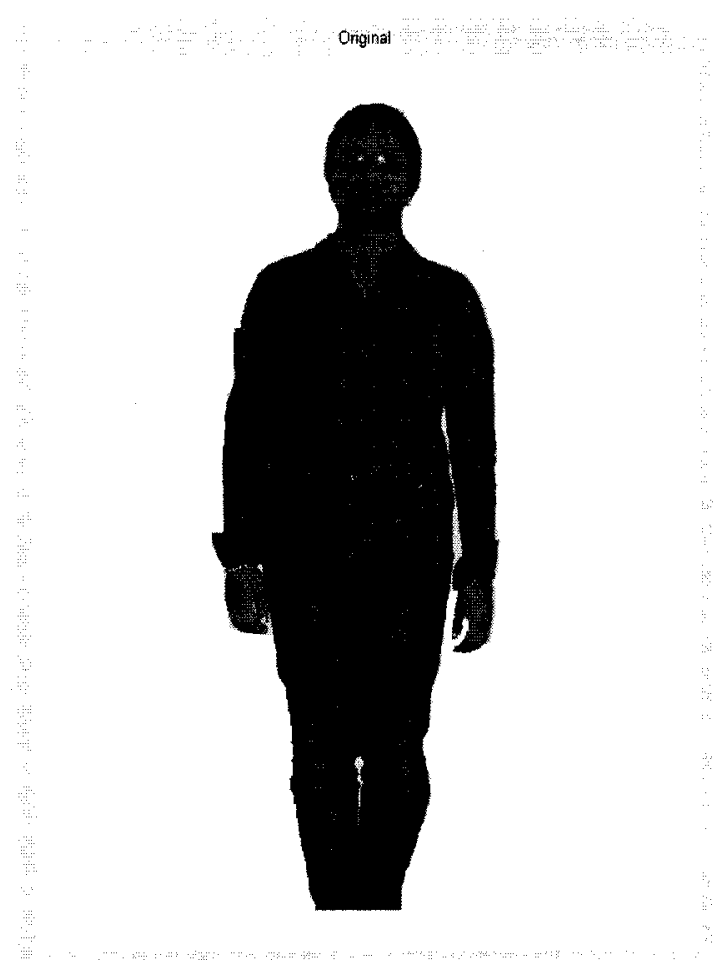

a Original Image

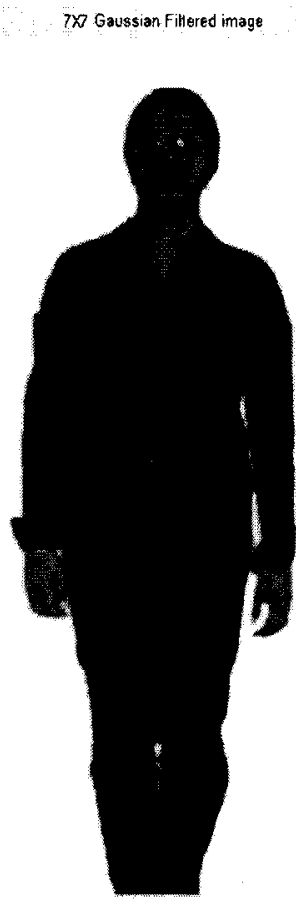

b Filtered Image

Figure 6: Original Image and Filtered Image 


\subsubsection{Edge Detection}

After filtering the images, the silhouette images are required to be found so that the background will be separated from the image. There are many ways to detect the edges in Matlab: Sobel, Prewitt, log, canny. They are used on all the filtered images: on original and noise added ones. A conclusion has been drawn from the result to get the better silhouette image.

Sobel edge detector does not detect all the edges with any of the five images. On the original image and median filtered salt and pepper noise image, it gives most of the edges. The result is almost the same for the Gaussian noisy image. On Gaussian filtered Gaussian noise, there are some edges detected. On salt and pepper noisy image, there are many white dots, so the edges are not clear at all.

Prewitt edge detector gives the same edge detection as Sobel edge detector for all five images.

The first two edge detectors are better than Log edge detector. On original image and median filtered salt and pepper noise image, it detects most of the edges but there is noise. On salt and pepper noisy image and Gaussian noisy image, there are many white dots, so the edges are not clear at all. On Gaussian filtered Gaussian noise image, it detects the edges but they are not clear at all. This edge detector is more sensitive to the noise. 
The first two edge detectors are better than Canny edge detector. On the original image and median filtered salt and pepper noise image, it does not detect all the edges but contains noise so the figure is not clear. On salt and pepper noisy image, Gaussian noisy image, and Gaussian filtered Gaussian noise image, there are many white dots, so the edges are not clear at all. This edge detector is more sensitive to the noise.

As a result, the Sobel edge detector has fulfilled the purpose of this project. Figure 7 shows the silhouette image, which is generated using Sobel. The other silhouette images using the other edge detectors are in Appendix A. 

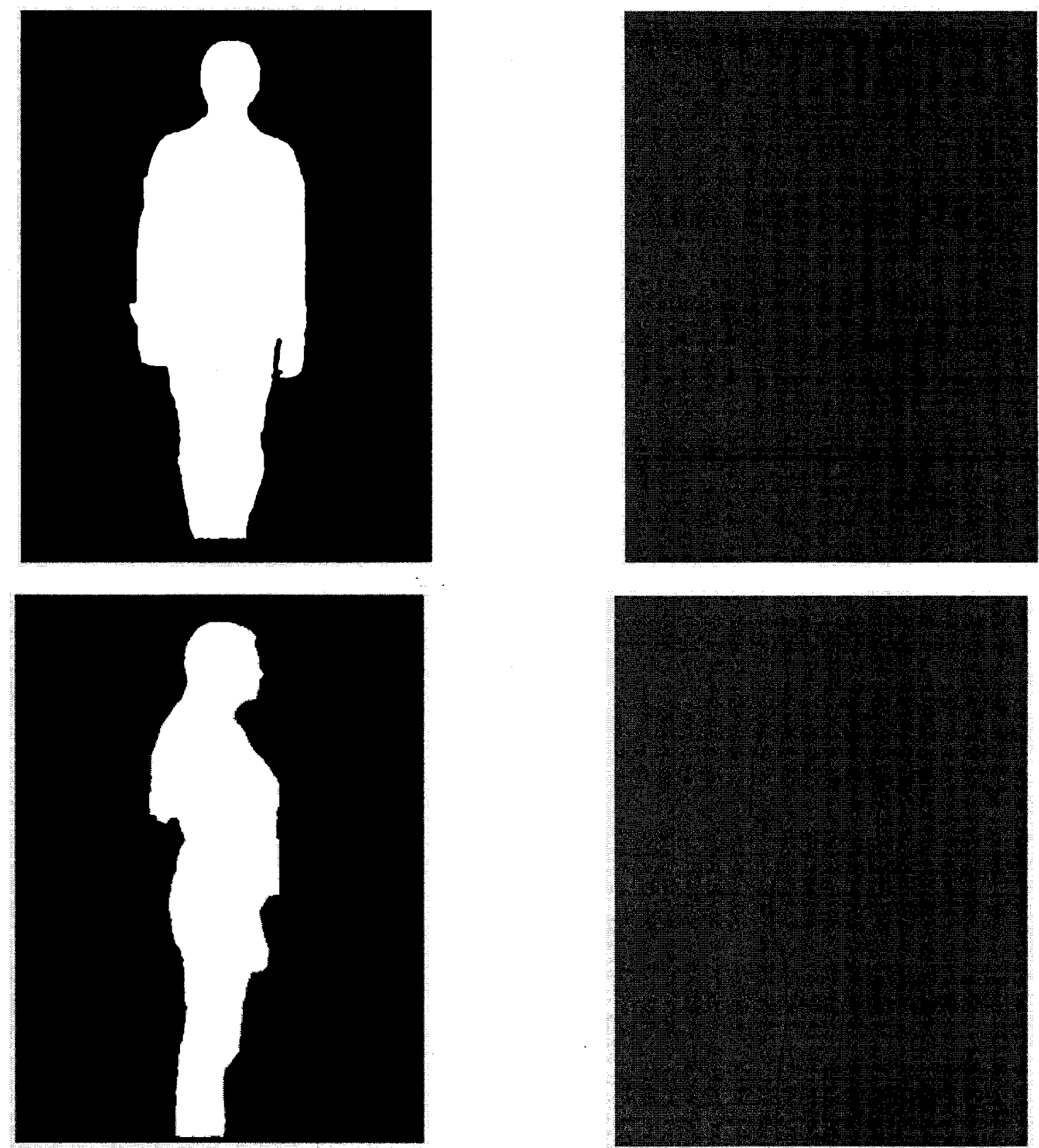

Figure 7: Silhouette Images of Front and Side View 


\subsubsection{Implementation of 3D Model Reconstruction}

MatLab is used to reconstruct a 3D model with the orthogonal images (front and side). The images are captured with a digital camera and are resized to $554 \times 738$ pixels. The reason for the resizing is because of the slowness of Matlab. Instead of going into all the details as discussed in the above sections, the $3 \mathrm{D}$ model is reconstructed using only two images for this project. Because the accuracy is needed for this specific system, the 3D model can be reconstructed with 2 orthogonal view images. The images are required to have white background for better output because Matlab gives good silhouette images if the background is white. The $3 \mathrm{D}$ humanoid model is also not used to implement this model. The reason for this is that the humanoid model is used mainly to create action figures from the reconstructed model. For this project's purpose the action figures are not needed at all.

Significant changes are made to the method to simplify the algorithm that is discussed above as it is implemented to reconstruct the 3D image. If the whole method is needed to be implemented, the time limitation will be the problem. Because of the time limitation, the method is simplified and used for the VDR system. Furthermore, this image can fulfill the purpose of this project. Figure 8 shows the resulted orthogonal 3D images. 

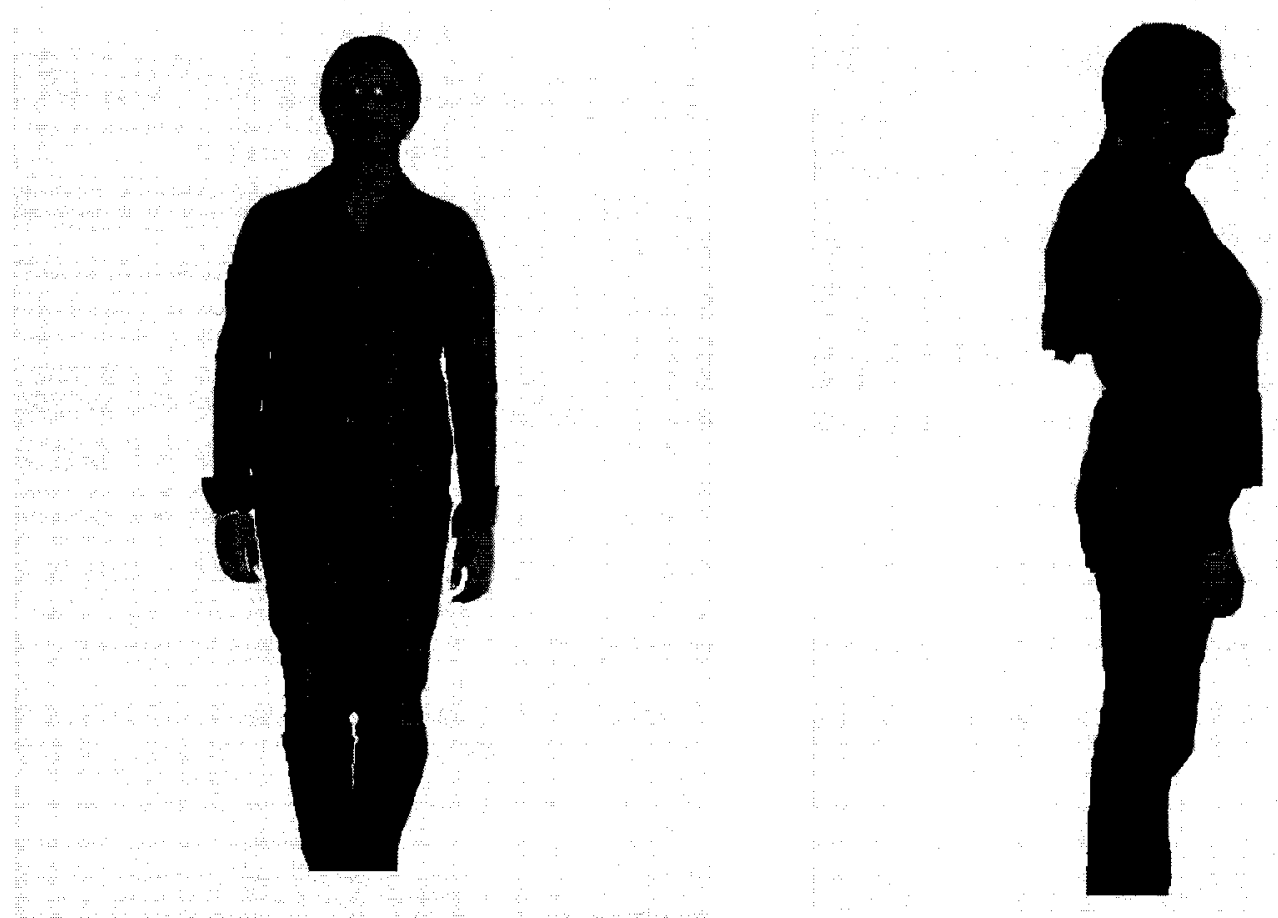

Figure 8: 3D Reconstructed Images 


\subsection{Neural Network Design}

Neural network was used to represent the network of biological neurons. But, now the term neural network used to refer artificial neural network, which is an interconnected group of artificial neurons or nodes. The neural network can be used in many fields to solve many problems that are impossible to solve by a computer technique.

Neural network can predict an answer, which is impossible to predicted by a human or a computer. "Neural networks, with their remarkable ability to derive meaning from complicated or imprecise data, can be used to extract patterns and detect trends that are too complex to be noticed by either humans or other computer techniques"[22]. Adaptive learning, self-organization, real time operation, and fault tolerance via redundant information coding are some of the advantages of neural network [21].

Neural network is used in medical purposes because of its accuracy. It is used for survival prediction for the cancer therapy. "Using just the TNM variables both the back propagation neural network and the probabilistic neural network are significantly more accurate..." [20]. It is also used for pattern recognition such as human face recognition [23]. Neural network can be used to solve real time problems such as signal processing or robotic navigation [24], [25], [26]-[29]. Using the penalty function method, a neural network proposed to solve nonlinear convex programming problems [30]. The same problem is solved using neural network with equality constraints [31] and linear equality constraints [32] 
In this project, the neural network is designed to predict a person's preference in cloths and styles. The preference of a person is not easy to predict. The neural network is trained with age, gender and ethnic groups. But, it is impossible to train the network with all the possibilities. After testing with some possible inputs, the rest of the perdition is required to leave with the network to decide.

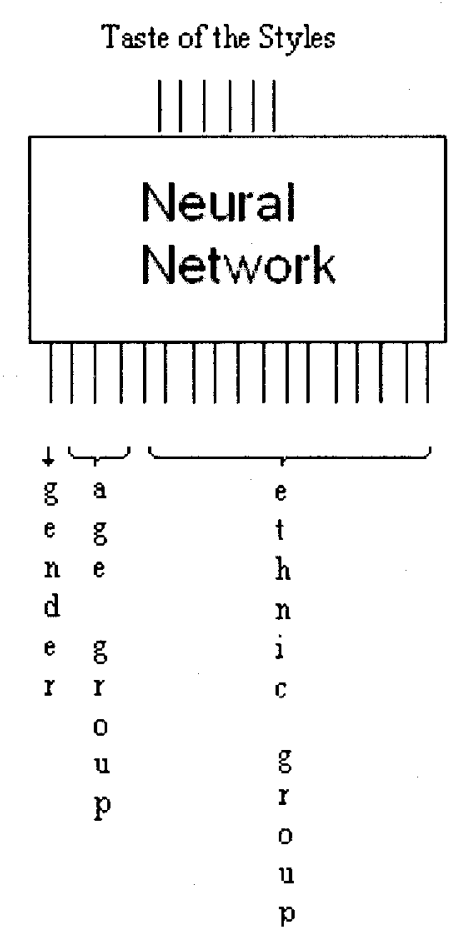

Figure 9: Architecture of the Neural Network

Designing and implementing a neural network will help the user to select clothes for their preference without going through the entire database. There are many ethnic groups in Canada and they have particular preference in different kinds if clothes. Age, gender and ethnic group will also affect the preference of the garments. 
This neural network is designed so that the network acquires age, gender and ethnic group as input strings and output the preference on a particular style.

There are 17 bits on the input string. The left most bit indicates the gender of the customer as shown in the table below.

Table 1: Input bit for gender

\begin{tabular}{|c|c|}
\hline Left most bit & Gender \\
\hline 0 & Female \\
\hline 1 & Male \\
\hline
\end{tabular}

Next 3 bits are for age groups. There will not be much different in the taste of the age 13 and 15 , but there will be a much different if the age is 13 and 23 . So the age is divided in to groups as shown in the table below.

Table 2: Input bits for age group

\begin{tabular}{|c|c|}
\hline Bits 2 to 4 & Age group \\
\hline $0,0,0$ & $6-12$ \\
\hline $0,1,0$ & Teenage \\
\hline $0,0,1$ & $20-25$ \\
\hline $0,1,1$ & $26-30$ \\
\hline $1,0,0$ & $31-40$ \\
\hline $1,1,0$ & $41-50$ \\
\hline $1,0,1$ & $51-65$ \\
\hline $1,1,1$ & Seniors \\
\hline
\end{tabular}


There are 13 ethnic groups in Canada. For this project, all the 13 ethnic groups are chosen. The digits 5 to 17 indicate the ethnic group. If the digit 5 is set, the British Isles origins ethnicity is chosen and if the digit 6 is set, the French origins ethnicity is chosen as shown in the table below.

Table 3: Input bits for ethnic group

\begin{tabular}{|c|l|}
\hline Bits 5 to 17 & \multicolumn{1}{|c|}{ Ethnic group } \\
\hline 5 & British Isles origins \\
\hline 6 & French origins \\
\hline 7 & Aboriginal origins \\
\hline 8 & North American origins \\
\hline 9 & Caribbean origins \\
\hline 10 & Latin/Central/South American origins \\
\hline 11 & European origins \\
\hline 12 & African origins \\
\hline 13 & Arab origins \\
\hline 14 & West Asian origins \\
\hline 15 & South Asian origins \\
\hline 16 & East and Southeast Asian origins \\
\hline 17 & Oceania origins \\
\hline & \\
\hline
\end{tabular}

The system trained with a customer from one particular ethic background. But the neural network will generalize and give the result if the customer has more then one ethnic background: American African, Asian American, and so on. 


\subsection{Implementations}

Two MLP networks are implemented using standard BP and LABP algorithms and they were tested with the inputs and outputs for this specific project. Visual $\mathrm{C}++$ is used to implement these algorithms. The system gets a string of binary numbers, which indicates the gender, age and ethnicity, as the inputs. The first bit indicates the gender: 0 is female and 1 is male. The last three bits indicates the age:000 is for age of 6-12, 010 is for teenage, 001 is for age between $20-25,011$ is for age between $26-30,100$ is for age between $31-40,110$ is for age between 41-50, 101 is for age between $51-65$ and 111 is for seniors. Next 13 bits are for ethnicities. The bits string, which is given to the input nodes, has seventeen bits. Then the system gives the preference of the six styles as the outputs.

\subsubsection{Implementation of Standard BP}

An MLP network is implemented using standard BP algorithm for this application. In this network, there is one hidden layer with hundred hidden neurons (nodes). Seventeen neurons in the input layer represent the seventeen bits in a training bits string. There are six nodes in the output layer. In this case, six output nodes are needed because it is going to return the taste of the six different styles. The training patterns contain all the combination of the age, gender and ethnic groups with seventeen bits. 
First, using rand() function, which is a built-in function in Visual $\mathrm{C}++$, the weights are initialized randomly. Then, net ${ }^{\mathrm{h}}$ is computed for all the hidden nodes by multiplying the inputs and the weights. Using net $^{\mathrm{h}}$ and sigmoid function, the output of the hidden nodes are calculated.

$$
\text { out_hidden }[i]=\operatorname{sigmoid}\left(\text { net }^{h}[i]\right)
$$

Further more, net ${ }^{\circ}$ is calculated for all the output nodes by multiplying the output of the hidden nodes and weights. Using net ${ }^{\circ}$ and sigmoid function, the actual outputs are calculated.

After computing the actual outputs, the error between the target and actual outputs are calculated using the following formula,

$$
E_{i}=y_{i}\left(1-y_{i}\right)\left(\hat{y}_{i}-y_{i}\right)
$$

Where, $\hat{y}_{i}$ is the desired output of the $\mathrm{i}^{\text {th }}$ output node and $\mathrm{y}_{\mathrm{i}}$ is the actual output of the $\mathrm{i}^{\text {th }}$ output node. Then the stopping factor is calculated using the error value.

$$
E P=0.5 \times \sum_{i=1}^{L} E_{i}^{2}
$$

That is the error from the entire $L$ output nodes (in this case $L=1$ ) are squared and added together. The half of this value is the stopping factor. This stopping factor is used later in the stopping condition to stop the training iteration. 
Using the error between the target and actual outputs, the hidden weight is calculated. The learning rate, error of the output and the outputs of the hidden nodes are multiplied together and added with the weight from the previous iteration to calculate the new hidden weight.

hidden_weight $[$ middlenode $][$ outputnode $]+=$ ETA $*$ error $[$ outputnode $] *$ out_hidden $[$ middlenode $]$

The error of the output node and the weights, which are connected to the output node, are multiplied with output of the hidden node as follows,

$$
E_{i} * w_{i j}^{o} * \text { out_hidden }[i] *(1-\text { out_hidden }[i])
$$

Then, it is multiplied with the input and the learning rate and added with the weight from the previous iteration to calculate new input weight.

The stopping factor (EP) is computed for the entire patterns and added together to get $\mathrm{EP}_{\text {sum. }}$ After every iteration, the new $\mathrm{EP}_{\text {sum }}$ is compared with the previous $\mathrm{EP}_{\text {sum. If }}$ the difference between those two $\mathrm{EP}_{\text {sum }}$ is smaller than a pre-specified tolerance, the training can be stopped because it means the neural network system is trained.

After the training, a set of test patterns is given as inputs to the MLP network. Using the weights, which were calculated by the training, the output bits are computed for all the test patterns. 


\subsubsection{Implementation of Modified-LABP}

Modified-LABP algorithm is implemented as the standard BP using Visual $\mathrm{C}++$. It differs from standard BP in some of the steps. For starter, LABP uses different equations to calculate the outputs (or activate the outputs).

$$
f_{j}(\bullet)=\frac{1}{1+e^{-\alpha(\bullet)}}
$$

The hidden weight is calculated as follows.

$$
\begin{aligned}
& \text { temp }=(\text { desired_output }[\text { symbol__index }][\text { outputnode }]-\text { out_output }[\text { outputnode }]) \\
& \text { hidden_weight_change }=\left(\frac{1}{\text { out_hidden }[\text { middlenode }]}\right) *\left(\frac{1}{\text { Total_hidden }}\right) * \text { temp }
\end{aligned}
$$

hidden_weight $[$ middlenode $][$ outputnode $]+=$ hidden_weight_change

where, the out_output represent the actual output, out_hidden represents the output of the hidden node, and Total_Hidden is the total number of nodes in the hidden layer. 
The input weight is calculated as follows.

input_weight_change $=((-1) *$ input_weight $[$ input_node $][$ middle_node $])+\left(\frac{1}{\text { TOTAL_INPUT }}\right)\left(\frac{1}{\text { input_pattern }[\text { symbol_index }][\text { input_node }]}\right)$

input_weight[input_node $][$ middle _node $]+=A L P H A^{*}$ della_hidden[middle_node $]^{*}$ input_pattern[symbol_index $][$ input_node $]$

where, input_weight represents the connection weight between input and hidden layers, TOTAL_INPUT represents the total number of nodes in the input layer, input pattern represents the inputs, and ALPHA is a pre-specified small number.

From the equations, you can see that the input weights are calculated almost like standard BP algorithm. This is not the way the LABP algorithm is proposed. These changes are made so that the Modified-LABP algorithm would be able to give better performances. The LABP is originally proposed for discrete time system. The inputs of the discrete time system are connected to each other and there is a small difference between two inputs. On the other hand, mapping function is used for this project's purpose and a mapping function does not have any connection between two inputs. This change makes the LABP algorithm to work on this system and gives a better result than standard BP algorithm. So this Modified-LABP is a better neural network for this project. 


\section{CHAPTER 4 - RESULTS AND DISCUSSION}

\subsection{Experimental Results}

First, both algorithms, standard BP and Modified-LABP are tested with an encoder.

The results by running the MLP neural network, which used standard BP algorithm, are presented below. 
Table 4: Iteration Table for Standard BP

\begin{tabular}{|l|l|}
\hline \multicolumn{2}{|l|}{ Iteration No. :1945 } \\
\hline DeltaEsum & 0.008001 \\
\hline BetaEsum & 0.000002 \\
\hline Iteration No. :1946 \\
\hline DeltaEsum & 0.007999 \\
\hline BetaEsum & 0.000002 \\
\hline
\end{tabular}

The Standard BP neural network is now being tested and following is the test result.

Table 5: Inputs and Outputs from Standard BP Algorithm

\begin{tabular}{|c|c|c|c|}
\hline & Input Pattern & Desired Output & Output \\
\hline 1 & 0000 & 0 & 0.444740 \\
\hline 2 & 0010 & 1 & 0.918392 \\
\hline 3 & 00001 & 1 & 0.910345 \\
\hline 4 & 0011 & 0 & 0.104549 \\
\hline 5 & 0100 & 1 & 0.915775 \\
\hline 6 & 0110 & 0 & 0.097797 \\
\hline 7 & 0101 & 0 & 0.138005 \\
\hline 8 & 01111 & 1 & 0.822363 \\
\hline 9 & 1000 & 1 & 0.921906 \\
\hline 10 & 1010 & 0 & 0.096581 \\
\hline 11 & 1001 & 0 & 0.101707 \\
\hline 12 & 1011 & 1 & 0.867716 \\
\hline 13 & 1100 & 0 & 0.091753 \\
\hline 14 & 1110 & 1 & 0.875901 \\
\hline 15 & 1101 & 1 & 0.837488 \\
\hline 16 & 1111 & 0 & 0.222477 \\
\hline
\end{tabular}


The output of the entire iteration is not given here, but the last two iterations and the test results are presented. As it is shown above, it took 1946 iteration to finish the training.

The results by running the MLP neural network, which used Modified-LASP algorithm, are presented below.

Table 6: Iteration Table for Modified-LABP

\begin{tabular}{|l|l|}
\hline \multicolumn{2}{|l|}{ Iteration No. :1451 } \\
\hline DeltaEsum & 0.008011 \\
\hline BetaEsum & 0.000011 \\
\hline Iteration No. :1452 \\
\hline DeltaEsum & 0.008000 \\
\hline BetaEsum & 0.000011 \\
\hline
\end{tabular}


The Modified-LABP neural network is now being tested and following is the test result.

Table 7: Inputs and Outputs from Modified-LABP Algorithm

\begin{tabular}{|c|c|c|c|}
\hline & Input Pattern & Desired Output & Output \\
\hline 1 & 0000 & 0 & 0.444740 \\
\hline 2 & 0010 & 1 & 0.918392 \\
\hline 3 & 00001 & 1 & 0.910345 \\
\hline 4 & 00011 & 0 & 0.104549 \\
\hline 5 & 0100 & 1 & 0.915775 \\
\hline 6 & 01110 & 0 & 0.097797 \\
\hline 7 & 0101 & 0 & 0.138005 \\
\hline 8 & 01111 & 1 & 0.822363 \\
\hline 9 & 1000 & 1 & 0.921906 \\
\hline 10 & 10010 & 0 & 0.096581 \\
\hline 11 & 1001 & 0 & 0.101707 \\
\hline 12 & 1011 & 1 & 0.867716 \\
\hline 13 & 1100 & 0 & 0.091753 \\
\hline 14 & 11110 & 1 & 0.875901 \\
\hline 15 & 1101 & 1 & 0.837488 \\
\hline 16 & 1111 & 0 & 0.222477 \\
\hline
\end{tabular}

The output of the entire iteration is not given here, but the last two iterations and the test results are presented. As it is shown above, it took only 1452 iteration to finish the training. It finished the training in 494 iterations earlier than standard BP algorithm, so it converges faster than standard BP. However, this algorithm works more effectively with discrete time system because the inputs of the discrete time system are connected to 
each other and there is a small difference between two inputs. On the other hand, this system is a mapping function, which does not have any connection between two inputs.

After the encoder testing, both algorithms, standard BP and Modified-LABP are tested for the VDR system. The garments are separated into groups of different styles. The inputs and desired outputs will be acquired from some customers to train the neural network. For this experiment, the assumption is that there are 6 styles of dresses. The outputs are chosen by the project developer and it does not have to be the actual liking of the particular group. The more the style groups leads to the more appropriate style for the particular age, gender and ethnic group. Because there is no actual database access, the project is tested with limited information.

The inputs are given in the following pattern:

Gender (1st bit)

0 -female

1-male

Age (last 3 bits)

$0,0,0-6-12$

0,1,0-teenage

$0,0,1-20-25$

$0,1,1-26-30$

$1,0,0-31-40$

$1,1,0-41-50$

1,0,1-51-65

$1,1,1$-seniors 
The rest of the digits (5 to 17) are for ethnic groups.

5 British Isles origins

6 French origins

7 Aboriginal origins

8 North American origins

9 Caribbean origins

10 Latin/Central/South American origins

11 European origins

12 African origins

13 Arab origins

14 west Asian origins

15 South Asian origins

16 East and Southeast Asian origins

17 Oceania origins

The output pattern indicates the style chosen by the users. The output 0.90 .00 .0 0.00 .00 .0 implies that they prefer style 1 ; the output 0.00 .90 .00 .00 .00 .0 means that they prefer style 2 , and the output 0.90 .80 .00 .00 .00 .0 means that they prefer style 1 and 2 , etc. When the age, gender and ethnic group are given as inputs, the neural network will output preference of styles. Then, the style number will be used to fetch the desired style garments' images from the database.

Both algorithms are trained with the same desired output. The Modified-LABP gave the correspondence results when it was tested as shown in Table 10, but the standard BP failed to do that. Table 10 shows a sample set of the inputs and the desired outputs. The ethnic group is British Isles origins because the $5^{\text {th }}$ digit is one. 
Table 8: A set of Sample Inputs and Desired Outputs

\begin{tabular}{|c|c|c|}
\hline & Input Pattern & Output- Styles \\
\hline & & $\begin{array}{llllll}1 & 2 & 3 & 4 & 5 & 6\end{array}$ \\
\hline 1 & 00001000000000000 & $\begin{array}{llllll}0.2 & 0.0 & 0.9 & 0.2 & 0.0 & 0.0\end{array}$ \\
\hline 2 & 00101000000000000 & $\begin{array}{llllll}0.0 & 0.9 & 0.0 & 0.8 & 0.0 & 0.0\end{array}$ \\
\hline 3 & 00011000000000000 & $\begin{array}{llllll}0.0 & 0.8 & 0.0 & 0.9 & 0.0 & 0.0\end{array}$ \\
\hline 4 & 00111000000000000 & $\begin{array}{llllll}0.0 & 0.8 & 0.0 & 0.9 & 0.0 & 0.0\end{array}$ \\
\hline 5 & 01001000000000000 & $\begin{array}{llllll}0.0 & 0.0 & 0.0 & 0.0 & 0.0 & 0.9\end{array}$ \\
\hline 6 & 01101000000000000 & $\begin{array}{llllll}0.0 & 0.0 & 0.0 & 0.0 & 0.0 & 0.9\end{array}$ \\
\hline 7 & 01011000000000000 & $\begin{array}{llllll}0.0 & 0.0 & 0.0 & 0.0 & 0.0 & 0.9\end{array}$ \\
\hline 8 & 01111000000000000 & $\begin{array}{llllll}0.0 & 0.0 & 0.0 & 0.0 & 0.0 & 0.8\end{array}$ \\
\hline 9 & 10001000000000000 & $\begin{array}{lllllll}0.9 & 0.0 & 0.0 & 0.0 & 0.0 & 0.0\end{array}$ \\
\hline 10 & 10101000000000000 & $\begin{array}{llllll}0.0 & 0.9 & 0.0 & 0.0 & 0.0 & 0.0\end{array}$ \\
\hline 11 & 10011000000000000 & $\begin{array}{llllll}0.0 & 0.9 & 0.0 & 0.0 & 0.0 & 0.0\end{array}$ \\
\hline 12 & 10111000000000000 & $\begin{array}{llllll}0.0 & 0.9 & 0.0 & 0.0 & 0.0 & 0.0\end{array}$ \\
\hline 13 & 11001000000000000 & $\begin{array}{llllll}0.0 & 0.0 & 0.0 & 0.0 & 0.9 & 0.0\end{array}$ \\
\hline 14 & 11101000000000000 & $\begin{array}{lllllll}0.0 & 0.0 & 0.0 & 0.0 & 0.9 & 0.0\end{array}$ \\
\hline 15 & 11011000000000000 & $\begin{array}{llllll}0.0 & 0.0 & 0.0 & 0.0 & 0.9 & 0.0\end{array}$ \\
\hline 16 & 11111000000000000 & $\begin{array}{llllll}0.0 & 0.0 & 0.0 & 0.0 & 0.9 & 0.0\end{array}$ \\
\hline
\end{tabular}


The results obtained by running the MLP neural network, which used Standard BP algorithm, are presented below.

Table 9: A set of Sample Inputs and Outputs from Standard BP Algorithm for VDR system

\begin{tabular}{|c|c|c|}
\hline & Input Pattern & Output- Styles \\
\hline & & 6 \\
\hline 1 & $000001 \ldots$ & 0.2020080 .0962070 .9983380 .3982130 .0004650 .000345 \\
\hline 2 & $0001001 \ldots$ & 0.0010990 .9963990 .0234800 .9744730 .0075660 .000027 \\
\hline 3 & $00011 \ldots$ & 0.0010830 .9912250 .0289170 .9726850 .0109440 .000026 \\
\hline 4 & $00111 \ldots$ & 0.0002310 .9963340 .0092970 .9819510 .0138300 .000032 \\
\hline 5 & $010001 \ldots$ & 0.0096120 .0032320 .0146360 .0089220 .0423560 .000012 \\
\hline 6 & $01101 \ldots$ & 0.0050250 .0039570 .0033040 .0222550 .0365270 .000007 \\
\hline 7 & $01011 \ldots$ & 0.0071580 .0027980 .0038580 .0263840 .0320750 .000005 \\
\hline 8 & $0 1 \longdiv { 1 1 1 \ldots }$ & 0.0016540 .0107730 .0039960 .0278440 .0209200 .000002 \\
\hline 9 & $10001 \ldots$ & 0.9596010 .0359500 .0220540 .0262630 .0379720 .000222 \\
\hline 10 & $10101 \ldots$ & 0.0338790 .9624600 .0028640 .0106480 .0210440 .000033 \\
\hline 11 & $10011 \ldots$ & 0.0282380 .9570440 .0119300 .0086510 .0260360 .000032 \\
\hline 12 & $10111 \ldots$ & 0.0021750 .9909770 .0073330 .0166160 .0297730 .000017 \\
\hline 13 & $110001 \ldots$ & 0.0115100 .0334320 .0114490 .0088480 .9398610 .000024 \\
\hline 14 & $11101 \ldots$ & 0.0099430 .0588950 .0077580 .0159720 .9369340 .000025 \\
\hline 15 & $11011 \ldots$ & 0.0115540 .0593460 .0065640 .0200690 .9391150 .000027 \\
\hline 16 & $11111 \ldots$ & 0.0068490 .0692310 .0047250 .0282860 .9176490 .000018 \\
\hline
\end{tabular}


The results obtained by running the MLP neural network, which used ModifiedLASP algorithm, are presented below.

Table 10: A set of Sample Inputs and Outputs from Modified-LABP Algorithm for VDR system

\begin{tabular}{|c|c|c|}
\hline \multirow{2}{*}{ 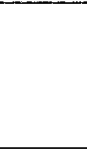 } & \multirow{2}{*}{ Input Pattern } & Output- Styles \\
\hline & & 4 \\
\hline 1 & $00001 \ldots$ & 0.4309180 .2935910 .9990020 .1377820 .0588610 .326631 \\
\hline 2 & $00101 \ldots$ & 0.0021340 .9986980 .0164970 .9820870 .0027080 .011313 \\
\hline 3 & $000011 \ldots$ & 0.0013270 .9979460 .0173430 .9820130 .0043400 .013336 \\
\hline 4 & $000111 \ldots$ & 0.0002470 .9983900 .0016680 .9944360 .0009430 .001734 \\
\hline 5 & $010001 \ldots$ & 0.0030960 .0047400 .0223840 .0063000 .0210570 .996422 \\
\hline 6 & $01101 \ldots$ & 0.0007920 .0138140 .0026680 .0083280 .0224170 .985913 \\
\hline 7 & $01011 \ldots$ & 0.0002600 .0122560 .0057090 .0121900 .0211790 .983870 \\
\hline 8 & $0111111 \ldots$ & 0.0002840 .0236340 .0023710 .0174990 .0168800 .975286 \\
\hline 9 & $10001 \ldots$ & 0.9719870 .0332400 .0230430 .0119740 .0257560 .000029 \\
\hline 10 & $10101 \ldots$ & 0.0199710 .9754570 .0020040 .0096850 .0100720 .000015 \\
\hline 11 & $100011 \ldots$ & 0.0198190 .9766090 .0021430 .0099930 .0116230 .000025 \\
\hline 12 & $10111 \ldots$ & 0.0037350 .9966350 .0017300 .0167330 .0068790 .000012 \\
\hline 13 & $11001 \ldots$ & 0.0186290 .0004430 .0023680 .0006300 .9778840 .013115 \\
\hline 14 & $11101 \ldots$ & 0.0042640 .0031010 .0014770 .0009250 .9808830 .015449 \\
\hline 15 & $11011 \ldots$ & 0.0038160 .0028840 .0017830 .0010720 .9804440 .015244 \\
\hline 16 & $11111 \ldots$ & 0.0044080 .0070340 .0011900 .0019290 .9687490 .016703 \\
\hline
\end{tabular}


Figure 10 shows the difference between the desired and the actual outputs. This is the result of Modified- LABP. There is not much error when there is less noise level. When the noise level increases, the error also increases. However, the error is still acceptable.

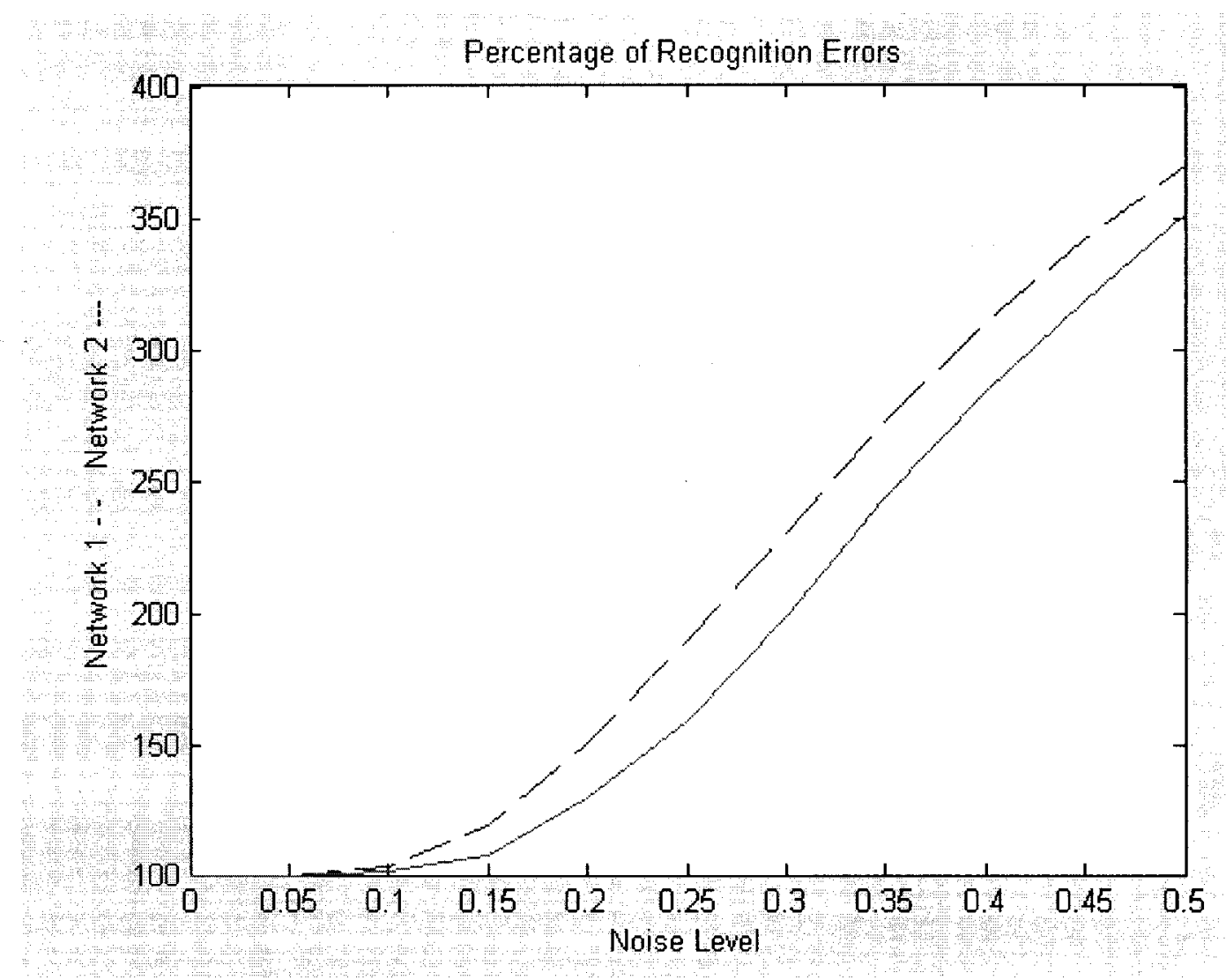

Figure 10: Desired and Actual outputs with added Noise level. 
Experiments have been performed to check if the system still works if one or more styles are removed.

Table 11: A set of Sample Inputs and Outputs from Modified-LABP Algorithm for VDR system with 4 Styles

\begin{tabular}{|l|l|l|}
\hline & Input Pattern & Output- Styles \\
\hline 1 & 00001000000000000 & 0.4309180 .9990020 .1377820 .058861 \\
\hline 2 & 00101000000000000 & 0.0021340 .0164970 .9820870 .002708 \\
\hline 3 & 000111000000000000 & 0.0013270 .0173430 .9820130 .004340 \\
\hline 4 & 0011110000000000000 & 0.0002470 .0016680 .9944360 .000943 \\
\hline 5 & 01001000000000000 & 0.0030960 .0223840 .0063000 .021057 \\
\hline 6 & 011010000000000000 & 0.0007920 .0026680 .0083280 .022417 \\
\hline 7 & 010111000000000000 & 0.0002600 .0057090 .0121900 .021179 \\
\hline 8 & 011111000000000000 & 0.0002840 .0023710 .0174990 .016880 \\
\hline 9 & 1000010000000000000 & 0.9719870 .0230430 .0119740 .025756 \\
\hline 10 & 1001010000000000000 & 0.0199710 .0020040 .0096850 .010072 \\
\hline 11 & 100111000000000000 & 0.0198190 .0021430 .0099930 .011623 \\
\hline 12 & 10111100000000000000 & 0.0037350 .0017300 .0167330 .006879 \\
\hline 13 & 11001000000000000 & 0.0186290 .0023680 .0006300 .977884 \\
\hline 14 & 111001000000000000 & 0.0042640 .0014770 .0009250 .980883 \\
\hline 15 & 11011000000000000 & 0.0038160 .0017830 .0010720 .980444 \\
\hline 16 & 111111000000000000 & 0.0044080 .0011900 .0019290 .968749 \\
\hline
\end{tabular}


Table 12: A set of Sample Inputs and Outputs from Modified-LABP Algorithm for VDR system with 5 Styles

\begin{tabular}{|l|l|l|l|}
\hline & Input Pattern & Output- Styles \\
\hline 1 & 00000100000000000000 & 0.4309180 .2935910 .9990020 .1377820 .058861 \\
\hline 2 & 000100100000000000000 & 0.0021340 .9986980 .0164970 .9820870 .002708 \\
\hline 3 & 00001100000000000000 & 0.0013270 .9979460 .0173430 .9820130 .004340 \\
\hline 4 & 0001111000000000000000 & 0.0002470 .9983900 .0016680 .9944360 .000943 \\
\hline 5 & 01000100000000000000 & 0.0030960 .0047400 .0223840 .0063000 .021057 \\
\hline 6 & 01110100000000000000 & 0.0007920 .0138140 .0026680 .0083280 .022417 \\
\hline 7 & 010101100000000000000 & 0.0002600 .0122560 .0057090 .0121900 .021179 \\
\hline 8 & 0111111000000000000000 & 0.0002840 .0236340 .0023710 .0174990 .016880 \\
\hline 9 & 10000100000000000000 & 0.9719870 .0332400 .0230430 .0119740 .025756 \\
\hline 10 & 100100100000000000000 & 0.0199710 .9754570 .0020040 .0096850 .010072 \\
\hline 11 & 10001100000000000000 & 0.0198190 .9766090 .0021430 .0099930 .011623 \\
\hline 12 & 101111000000000000000 & 0.0037350 .9966350 .0017300 .0167330 .006879 \\
\hline 13 & 11000100000000000000 & 0.0186290 .0004430 .0023680 .0006300 .977884 \\
\hline 14 & 111100100000000000000 & 0.0042640 .0031010 .0014770 .0009250 .980883 \\
\hline 15 & 111011100000000000000 & 0.0038160 .0028840 .0017830 .0010720 .980444 \\
\hline 16 & 1111111000000000000000 & 0.0044080 .0070340 .0011900 .0019290 .968749 \\
\hline
\end{tabular}


The experiment is repeated using more styles as outputs as shown in the tables below. The first table shows the desired outputs; whereas, the rest show the results using Modified-LABP. The reason for this extended experiment is to check if the system still works if more styles are introduced and whether it will adjust accordingly. The test was done using many outputs out of which, 8 have been shown.

Table 13: A set of Sample Inputs and Desired Outputs for 7 Styles

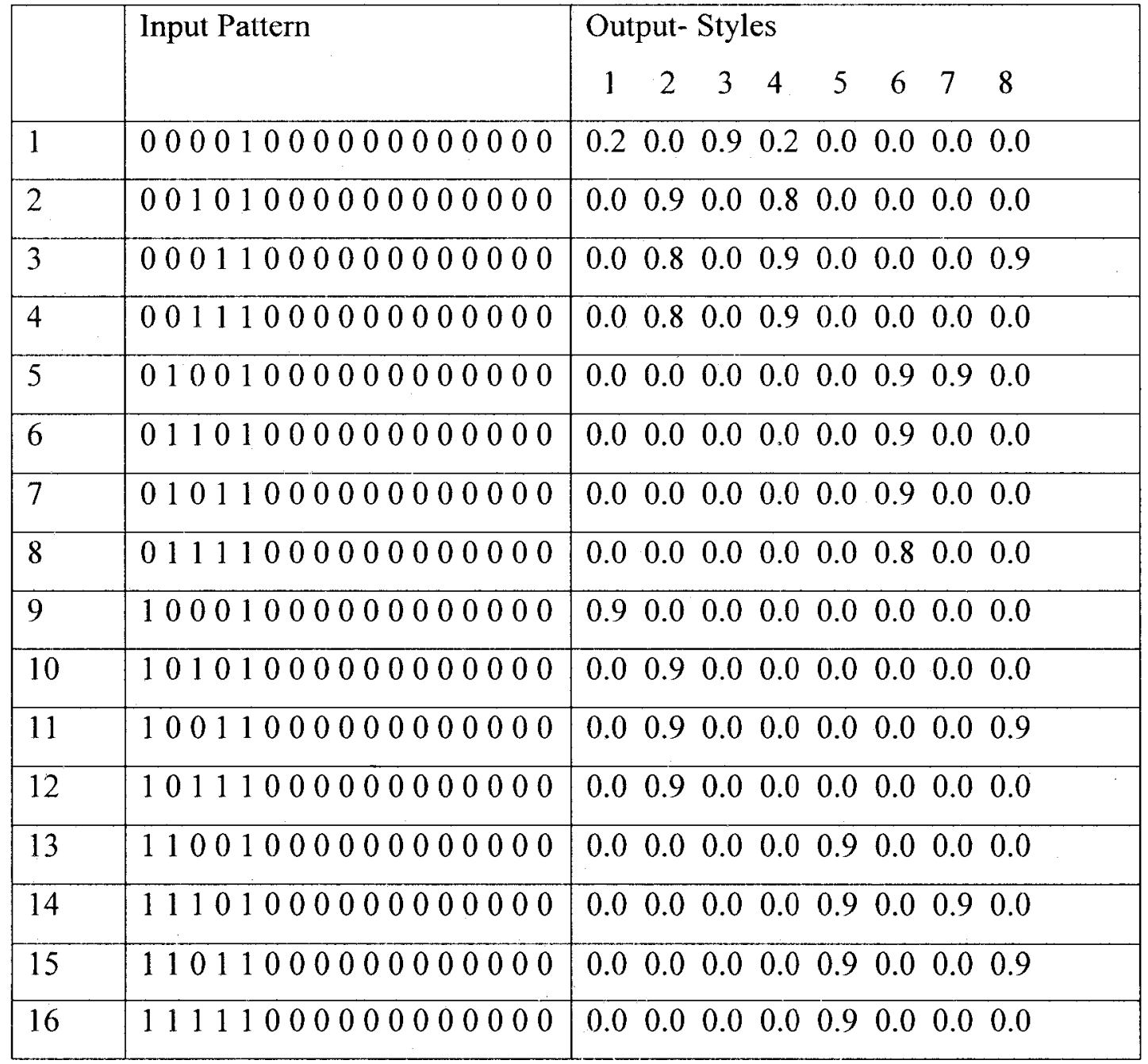


Table 14: A set of Sample Inputs and Outputs from Modified-LABP Algorithm for VDR system with 7 Styles

\begin{tabular}{|c|c|c|}
\hline & \multirow{2}{*}{$\begin{array}{l}\text { Input } \\
\text { Pattern }\end{array}$} & Output- Styles \\
\hline & & 7 \\
\hline 1 & $000001 \ldots$ & 0.4309180 .2935910 .9990020 .1377820 .0588610 .3266310 .023043 \\
\hline 2 & $00101 \ldots$ & 0.0021340 .9986980 .0164970 .9820870 .0027080 .0113130 .003735 \\
\hline 3 & $000011 \ldots$ & 0.0013270 .9979460 .0173430 .9820130 .0043400 .0133360 .023712 \\
\hline 4 & $000111 \ldots$. & 0.0002470 .9983900 .0016680 .9944360 .0009430 .0017340 .019621 \\
\hline 5 & $01001 \ldots$ & 0.0030960 .0047400 .0223840 .0063000 .0210570 .9964220 .998390 \\
\hline 6 & $01101 \ldots$ & 0.0007920 .0138140 .0026680 .0083280 .0224170 .9859130 .015251 \\
\hline 7 & $0101011 \ldots$ & 0.0002600 .0122560 .0057090 .0121900 .0211790 .9838700 .001477 \\
\hline 8 & $01111 \ldots$ & 0.0002840 .0236340 .0023710 .0174990 .0168800 .9752860 .137782 \\
\hline 9 & $10001 \ldots$ & 0.9719870 .0332400 .0230430 .0119740 .0257560 .0000290 .004264 \\
\hline 10 & $10101 \ldots$ & 0.0199710 .9754570 .0020040 .0096850 .0100720 .0000150 .013096 \\
\hline 11 & $10011 \ldots$ & 0.0198190 .9766090 .0021430 .0099930 .0116230 .0000250 .032688 \\
\hline 12 & $10111 \ldots$ & 0.0037350 .9966350 .0017300 .0167330 .0068790 .0000120 .016997 \\
\hline 13 & 11001 & 0.0186290 .0004430 .0023680 .0006300 .9778840 .0131150 .005540 \\
\hline 14 & $11101 \ldots$ & 0.0042640 .0031010 .0014770 .0009250 .9808830 .0154490 .986635 \\
\hline 15 & $11011 \ldots$ & 0.0038160 .0028840 .0017830 .0010720 .9804440 .0152440 .006301 \\
\hline 16 & $11111 \ldots$ & 0.0044080 .0070340 .0011900 .0019290 .9687490 .0167030 .013715 \\
\hline
\end{tabular}


Table 15: A set of Sample Inputs and Outputs from Modified-LABP Algorithm for VDR system with 8 Styles

\begin{tabular}{|c|c|c|}
\hline & Input & Output- Styles \\
\hline & Pattern & 7 \\
\hline 1 & $00001 \ldots$ & 0.4309180 .2935910 .9990020 .1377820 .0588610 .3266310 .0230430 .032546 \\
\hline 2 & $0010101 \ldots$ & 0.0021340 .9986980 .0164970 .9820870 .0027080 .0113130 .0037350 .024568 \\
\hline 3 & $00011 \ldots$ & 0.0013270 .9979460 .0173430 .9820130 .0043400 .0133360 .0237120 .922243 \\
\hline 4 & $0001111 \ldots$ & 0.0002470 .9983900 .0016680 .9944360 .0009430 .0017340 .0196210 .019530 \\
\hline 5 & $010001 \ldots$ & 0.0030960 .0047400 .0223840 .0063000 .0210570 .9964220 .9983900 .001241 \\
\hline 6 & $01101 \ldots$ & 0.0007920 .0138140 .0026680 .0083280 .0224170 .9859130 .0152510 .015466 \\
\hline 7 & $01011 \ldots$ & 0.0002600 .0122560 .0057090 .0121900 .0211790 .9838700 .0014770 .017034 \\
\hline 8 & $011111 \ldots$ & 0.0002840 .0236340 .0023710 .0174990 .0168800 .9752860 .1377820 .125758 \\
\hline 9 & $10001 \ldots$ & 0.9719870 .0332400 .0230430 .0119740 .0257560 .0000290 .0042640 .128698 \\
\hline 10 & $10101 \ldots$ & 0.0199710 .9754570 .0020040 .0096850 .0100720 .0000150 .0130960 .016311 \\
\hline 11 & $100011 \ldots$ & 0.0198190 .9766090 .0021430 .0099930 .0116230 .0000250 .0326880 .916191 \\
\hline 12 & $101111 \ldots$ & 0.0037350 .9966350 .0017300 .0167330 .0068790 .0000120 .0169970 .118321 \\
\hline 13 & $11001 \ldots$ & 0.0186290 .0004430 .0023680 .0006300 .9778840 .0131150 .0055400 .017443 \\
\hline 14 & $11101 \ldots$ & 0.0042640 .0031010 .0014770 .0009250 .9808830 .0154490 .9866350 .004340 \\
\hline 15 & $11011 \ldots$ & 0.0038160 .0028840 .0017830 .0010720 .9804440 .0152440 .0063010 .916225 \\
\hline 16 & $11111 \ldots$ & 0.0044080 .0070340 .0011900 .0019290 .9687490 .0167030 .0137150 .114720 \\
\hline
\end{tabular}


A test was performed to check the neural networks generalization technique. If a person has more than one ethnic background the network is required to generalize the style and give an appropriate preference as output.

Table 16: A Sample Generalization Result

\begin{tabular}{|c|c|c|c|c|c|c|c|c|}
\hline Gender & $\begin{array}{l}\text { Age } \\
\text { Group }\end{array}$ & $\begin{array}{l}\text { Ethnic } \\
\text { Group }\end{array}$ & $\begin{array}{l}\text { Input } \\
\text { (Sample) }\end{array}$ & $\begin{array}{l}\text { Output } \\
1\end{array}$ & 3 & 4 & 5 & 6 \\
\hline Female & $26-30$ & British Isles origins & $00011110 \ldots$ & 0.00 .8 & 0.0 & 0.9 & 0.0 & 0.0 \\
\hline Female & $26-30$ & French origins & $001101 \ldots$ & $0.0 \quad 0.0$ & 0.9 & 0.9 & 0.0 & 0.0 \\
\hline Female & $26-30$ & $\begin{array}{l}\text { British Isles and } \\
\text { French origins }\end{array}$ & $001111 \ldots$ & 0.00 .4 & 0.5 & 0.9 & 0.0 & 0.0 \\
\hline
\end{tabular}

\subsection{Comments}

Many algorithms were proposed to accelerate the MLP training algorithm. This report discusses two of these algorithms: Lyapunov stability-based adaptive backpropagation algorithm (LABP) and Recursive least squares algorithm (RLS). LABP guarantees the stability of the total network. The error between target and actual outputs converges to zero asymptotically. RLS guarantees fast convergence without trap in the local minima.

For this project, standard BP and Modified-LABP algorithms are implemented to use in Virtual Dressing Room (VDR) system using Visual C++. First both algorithms are 
tested with an encoder and the Modified-LABP algorithm finished the training 494 iteration advanced to the standard BP. This algorithm is proposed for discrete time system. However, the changes in the process of updating the input weights make the Modified-LABP algorithm to work for the encoder. The results show that ModifiedLABP works better than standard back-propagation algorithm. Then both of them are tested with the age, gender and ethnic group as inputs and preference of styles as outputs. The Modified-LABP gives the better output and the standard BP does not give the correct output at all. This shows that the standard BP cannot be used in the VDR system. It may give the correct output, if the correct learning rate is found; but that is almost impossible. Also, if there is a new style in the store, the neural network must be trained again with the new desired outputs. In that case, finding the learning rate again is not practical. Therefore, using Modified-LABP is the most appropriate for this project because one does not have to change anything other than the desired output for Modified-LABP.

The results also show that any number of styles can be added and removed from the system and the system will predict the output. Furthermore, the customer can have any ethnic group or can have any number of ethnic backgrounds. The system will generalize the output and predict the correct results. 


\section{CHAPTER 5 - CONCLUSION AND FUTURE WORK}

The clothing stores' customer friendly environment is increased by this project: Virtual Dressing Room (VDR) simulation. The 3D reconstruction program is implemented to acquire photos of a customer and reconstruct a 3D model of the particular customer, so the customer can try the garments without physically 'put on' them. Moreover, choosing the appropriate garments for a particular group of customers using a Modified-LABP MLP neural network is implemented. The customer, if she or he wanted, leaves the system to select garments that are of interest to their particular age, gender and ethnic group. It will save their time because the database will have thousands of garments for both genders and there will be sports clothing and wedding dresses too. If a customer has to go through the entire database to select an item it is waste of time. However, if they do not want the garments that are selected by the system for them, they 
can always fetch the entire garments form the database to try. Furthermore, the user interface for the system is created so the customer can easily use the VDR system.

Moreover, the system is built in a way so the management can add or remove any number of styles to the system and it will work correctly. Also, any number of inputs can be added.

\subsection{Future Work}

There is some work remaining to be done to use the Virtual Dressing Room (VDR) system as full. First, the application must read the images directly from the cameras and save them. Because of the lack of equipment and software, this is not implemented yet. Now the VDR system uses already-taken images and no cameras are used.

A random number, to be used as an access password, must be created for each $3 \mathrm{D}$ reconstructed image. The DSM method and merging the garments and the $3 \mathrm{D}$ images remain to be implemented in future work.

The current implementation of the neural network has as inputs: gender, age, and ethnic groups and as output numbers to indicate the preference of styles. This must be 
changed so that shape, and skin complexion will be included in inputs and size and color will be included in outputs. The VDR system also needs to get an appropriate database to check the Modified-LABP. 


\section{REFERENCE}

[1] A. Hilton, D.Beresford, T. Gentils, R.J.Smith, W. Sun, and J.Illingworth, "WholeBody Modelling of People from Multi-View Images to Populate Virtual Worlds," Journal the vision computer, vol. 16, pp. 411-436, Nov. 2000.

[2] R. Cipolla and P. Giblin, "Visual Motion of Curves and Surfaces" Cambridge University Press, 2000.

[3] C. Zhao and R. Mohr, "B-spline Patches for Surface Reconstruction in Computer Vision," In P.-J. Laurent, A. L. Mehaute, and L. Schumaker, editors, Wavelets, Images and Surface Fitting, pp 521-528, 1994.

[4] G. Cross and A. W. Zisserman, "Quadric Surface Reconstruction from Dual-Space Geometry," In Proceedings of the $6^{\text {th }}$ International Conference on Computer Vision, $\mathrm{pp}$ 25-31, 1998.

[5] C. C. Slama, editor, "Manual of Photogrammetry. American Society of Photogrammetry and Remote Sensing," Falls Church, Virginia, USA, 1980.

[6] S. Sullivan and J. Ponce, "Automatic Model Construction and Pose Estimation from Photographs using Triangular Splines," IEEE Transactions on Pattern Analysis and Machine Intelligence, vol. 20(10), pp1091-1097, 1998.

[7] P. Fua and Y. LeClerc, "Object-Centered Surface Reconstruction: Combining MultiImage Stereo and Shading," International Journal of Computer Vision, vol. 16, pp35-56, 1995.

[8] L. Zhang and S. Seitz, "Image-Based Multiresolution Shape Recovery by Surface Deformation," In Proceedings of SPIE: Videometrics and Optical Methods for 3D Shape Measurement, pp 51-61, 2001.

[9] Chang Shu and Gerhard Roth, "Free-From Surface Reconstruction from Multiple Images," 4th International Conference on 3-D Digital Imaging and Modeling, pp163-170, October, 2003.

[10]Y. 'Zhuang C. Shieh T. Liang and C. Chou, "Maximizing Speedup through Performance Prediction for Distributed Shared Memory Systems," IEEE $21^{\text {st }}$ International Conference on Distributed Computing Systems, pp.723-726, April 2001.

[11] Y. Roh Byeong, H. Seong and D. Park, "Hiding Latency Through Bulk Transfer and Prefetching in Distributed Shared Memory Multiprocessors," IEEE $4^{\text {th }}$ International Conference on High Performance Computing, vol. 1, pp. 164-166, May 2000. 
[12] M. Kistler, and L. Alvisi, "Improving the Performance of Software Distributed Shared Memory with Speculation," IEEE Transactions on Parallel and Distributed Systems, vol. 16, pp. 885-896, September 2005.

[13] A. Kodi, and A. Louri, "RAPID: Reconfigurable and Scalable All-Photonic Interconnect for Distributed Shared Memory Multiprocessors," IEEE Journal Lightwave Technology, vol. 22, pp. 2101-2110, September 2004.

[14] T. Trevisan, V. Costa, L. Whately, and C. Amorim, "Distributed Shared Memory in Kernel Mode," Proceedings of IEEE $14^{\text {th }}$ Symposium Computer Architecture and High Performance Computing, pp. 159-166, October 2002.

[15] Z. Man, S. Phooi, and H. Wu, "Lyapunov Stability-Based Adaptive Backpropagation for Discrete Time System," Proceedings of IEEE Fifth Int. Symposium Signal Processing and Its Applications, vol. 2, pp. 661-664, Aug. 1999.

[16] A. Parlos, B. Fernandez, A. Atiya, J. Muthusami, and W. Tsai, "An Accelerated Learning Algorithm for Multilayer Perceptron Networks," IEEE Transactions on Neural Networks, vol. 5, pp. 493-497, May 1994.

[17] J. Bilski, and L. Rutkowski, "A Fast Training Algorithm for Neural Networks," IEEE Transactions on Circuits and Systems II: Analog and Digital Signal Processing, vol. 45, pp. 749-753, Jun. 1998.

[18] N. Jojic, J. Gu, T.S. Shen, and T.S Huang, "Computer Modeling, Analysis, and Synthesis of Dressed Humans," IEEE Transactions on Circuits and Systems for Video Technology, vol.9, pp.378 - 388, Mar. 1999.

[19] A. Sappa, N. Aifanti, S. Malassiotis, and M.G. Strintzis, "Monocular 3D Human Body Reconstruction Towards Depth Augmentation of Television Sequences," International Conference on Image Processing, 2003. ICIP 2003. Proceedings. 2003, vol. 2, pp. 325-328, Sept. 2003.

[20] H.B.Burke, D.B.Rosen, and P.H.Goodman, "Comparing Artificial Neural Networks to Other Statistical Methods for Medical Outcome Prediction," IEEE International Conference on Computational Intelligence and IEEE World Congress on Neural Networks, vol. 4, pp. 2213 - 2216, July 1994.

[21] I.Aleksander, and H.Morton, "An Introduction to Neural Computing," 2nd edition [22] C. Stergiou and D. Siganos, "Neural Networks." http://www.doc.ic.ac.uk/ nd/surprise_96/journal/vol4/cs11/report.html\#References [23] L.Chen, "Pattern Classification by Assembling Small Neural Networks," IEEE International Conference on Neural Networks, vol. 3, pp.1947-1952, July 2005. 
[24] T. Yoshikawa, "Foundations of Robotics: Analysis and Control," Cambridge, MA: MIT Press, 1990.

[25] B. Kosko, "Neural Networks for Signal Processing," Englewood Cliffs, NJ:

Prentice- Hall, 1992.

[26] D. W. Tank and J. J. Hopfield, "Simple Neural Optimization Networks: An A/D Converter, Signal Decision Circuit, and a Linear Programming Circuit," IEEE Transactions on Circuits Syst. II, vol. 33, pp. 533-541, May 1986.

[27] A. Cichocki and R. Unbehauen, "Switched-Capacitor Artificial Neural Networks for Differential Optimization,” J. Circuit Theory Applicat., vol.19, pp. 161-187, 1991.

[28] G. L. Dempsey and E. S. McVey, "Circuit Implementation of a Peak Detector Neural Network," IEEE Transactions on Circuits Syst. II, vol. 40, pp. 585-591, Sept. 1993.

[29] A. Cichocki and R. Unbehauen, "Neural Networks for Optimization and Signal Processing," New York: Wiley, 1993.

[30] M.P.Kennedy and L.O.Chua, "Neural Networks for Nonlinear Programming," IEEE Transactions on Circuits System II, vol. 35, pp. 554-562, May 1988.

[31] S. Zhang, X. Zhu, and L.-H. Zou, "Second-Order Neural Networks for Constrained Optimization," IEEE Transactions on Neural Networks, vol. 3, pp. 1021-1024, Nov. 1992.

[32] Q. Tao, J. D. Cao, M. S. Xue, and H. Qiao, "A High Performance Neural Network for Solving Nonlinear Programming Problems with Hybrid Constraints," Phys. Lett. A, vol. 288, no. 2, pp. 88-94, 2001. 


\section{APPENDIX A - THE RESULTED IMAGES}

The images show the different results acquired from the different filters and edge detection.

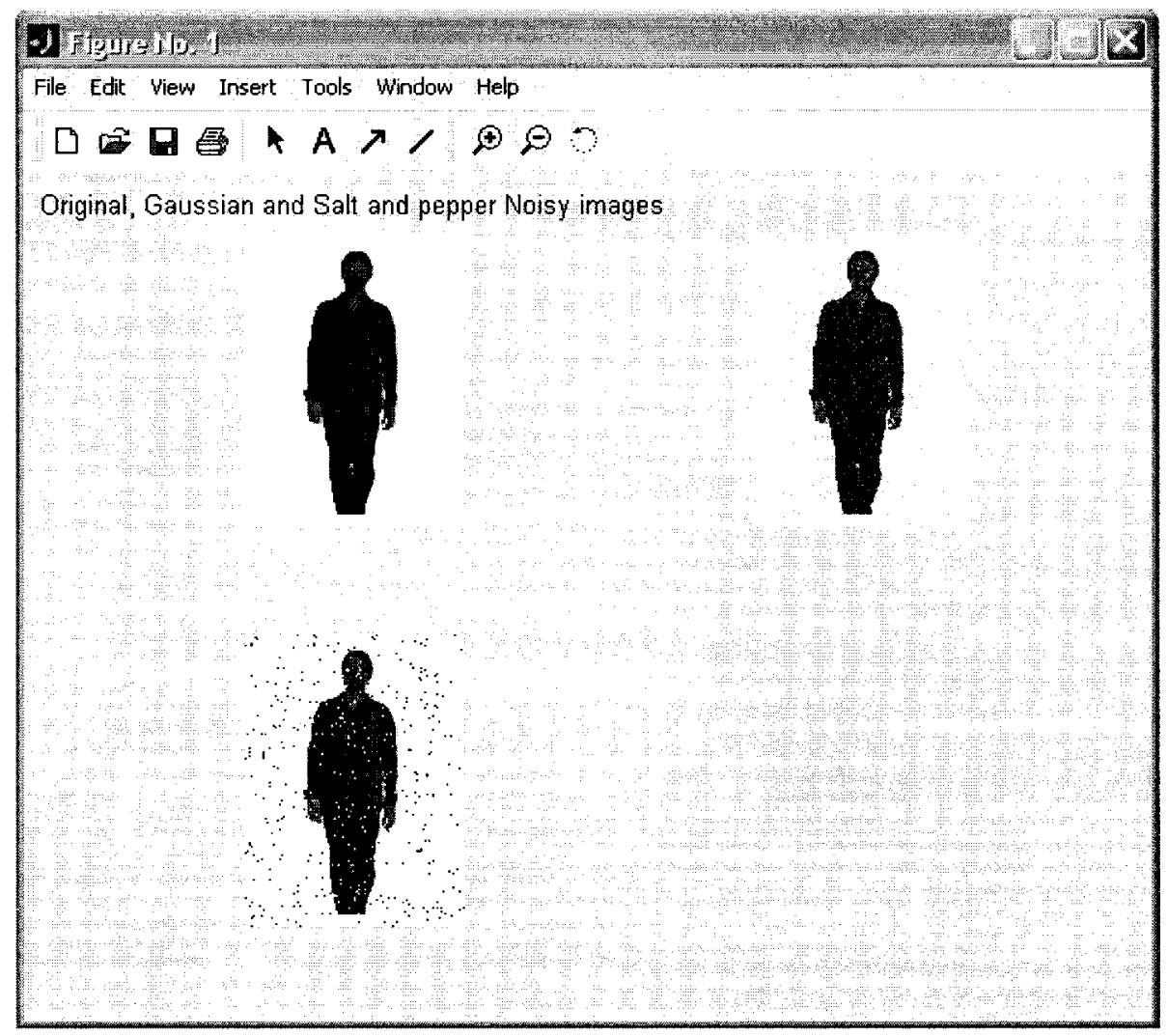

Figure 11: Original and Gaussian and Salt and Pepper Noisy Images 


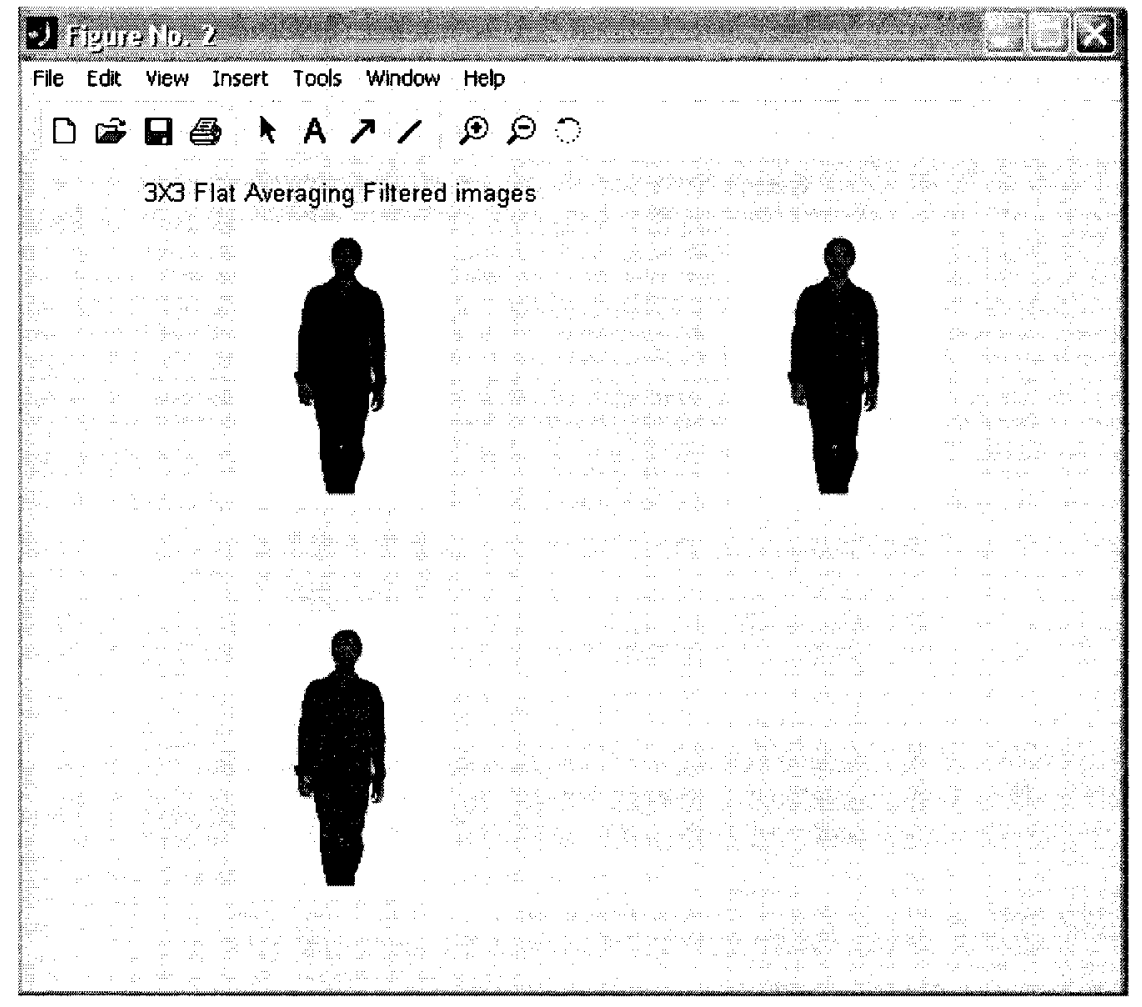

Figure 12: 3X3 Flat Averaging Filtered Images

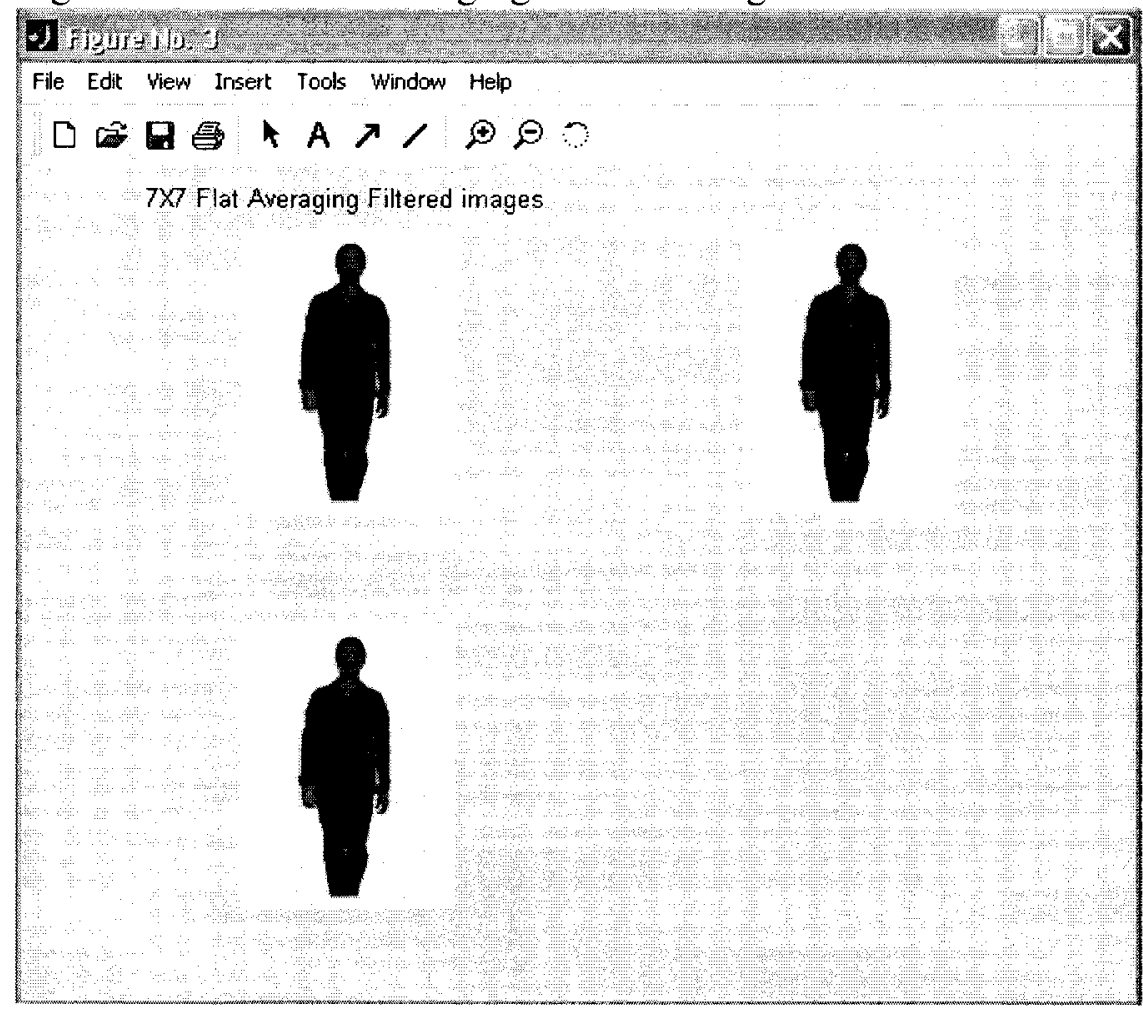

Figure 13: 7X7 Flat Averaging Filtered Images 


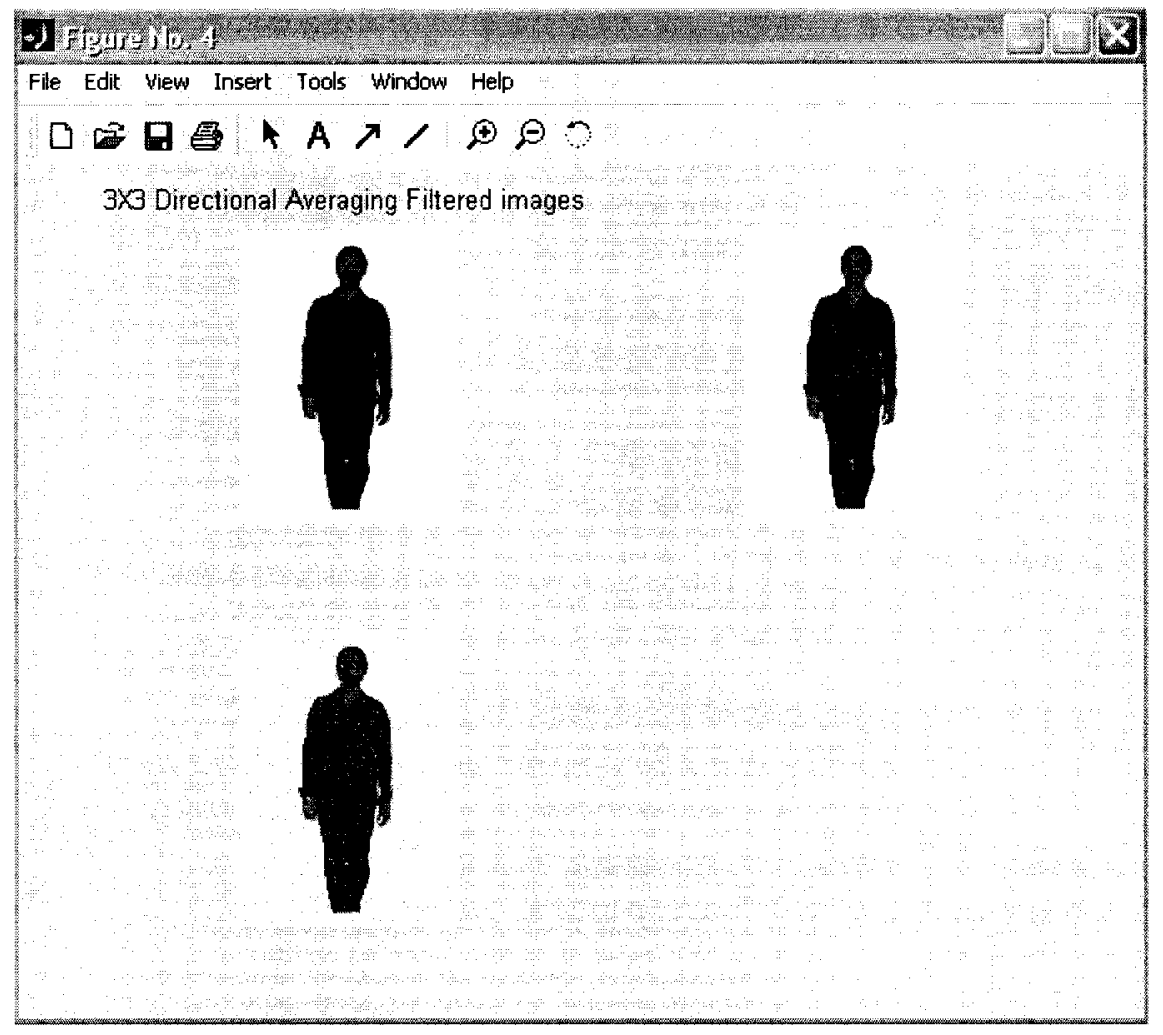

Figure 14: 3X3 Directional Averaging Filtered Images

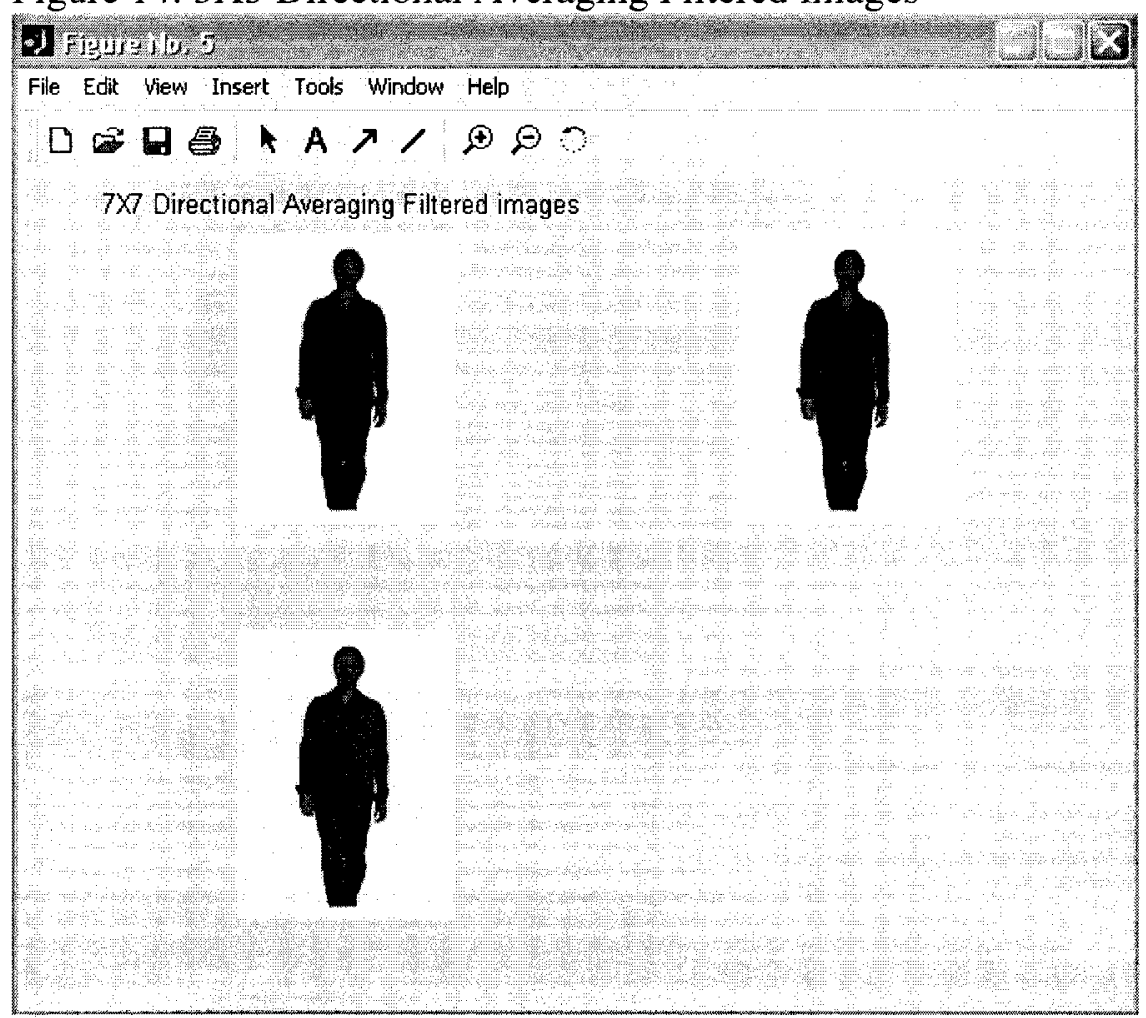

Figure 15: 7X7 Directional Averaging Filtered Images 


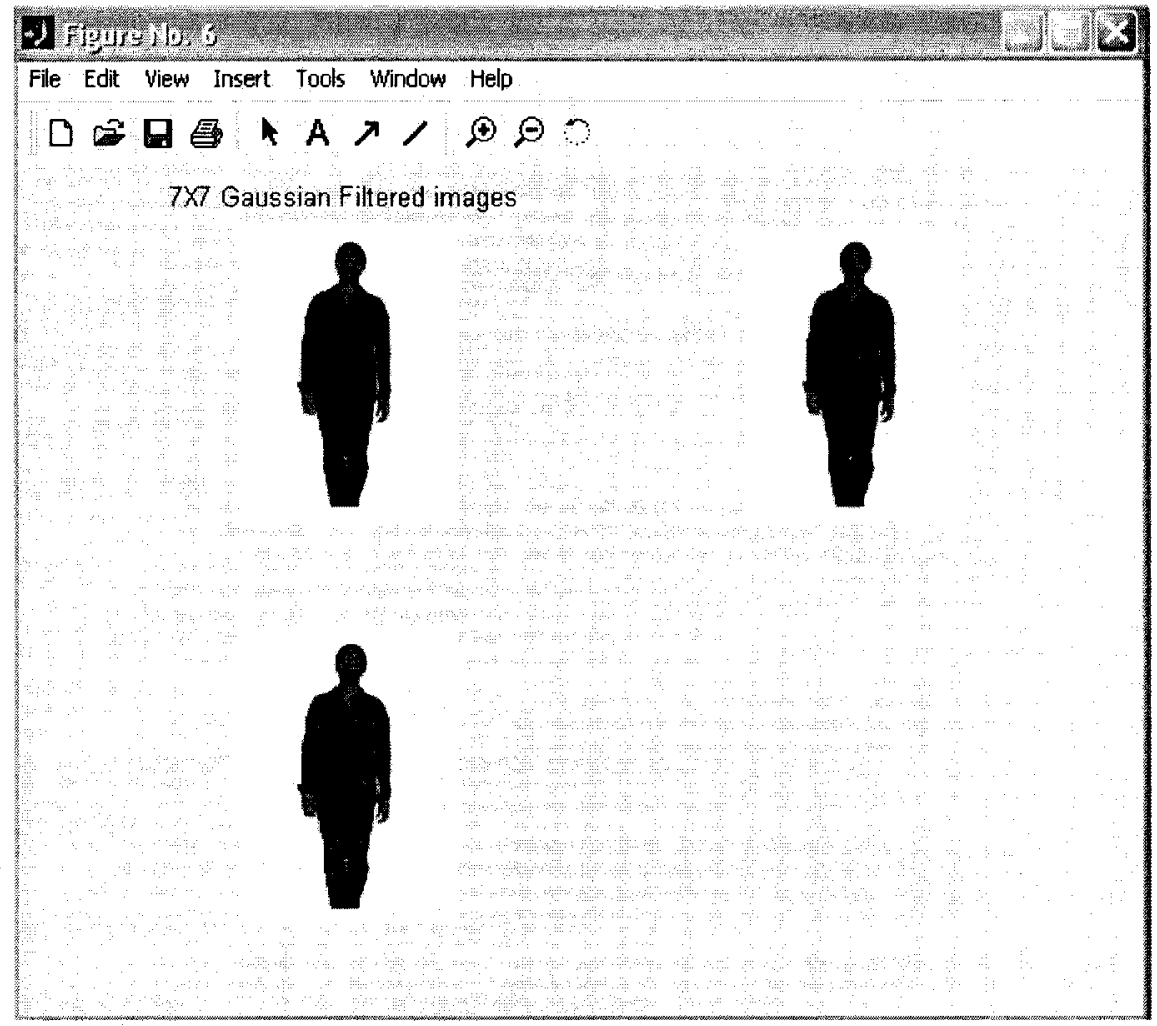

Figure 16: 7X7 Gaussian Filtered Images

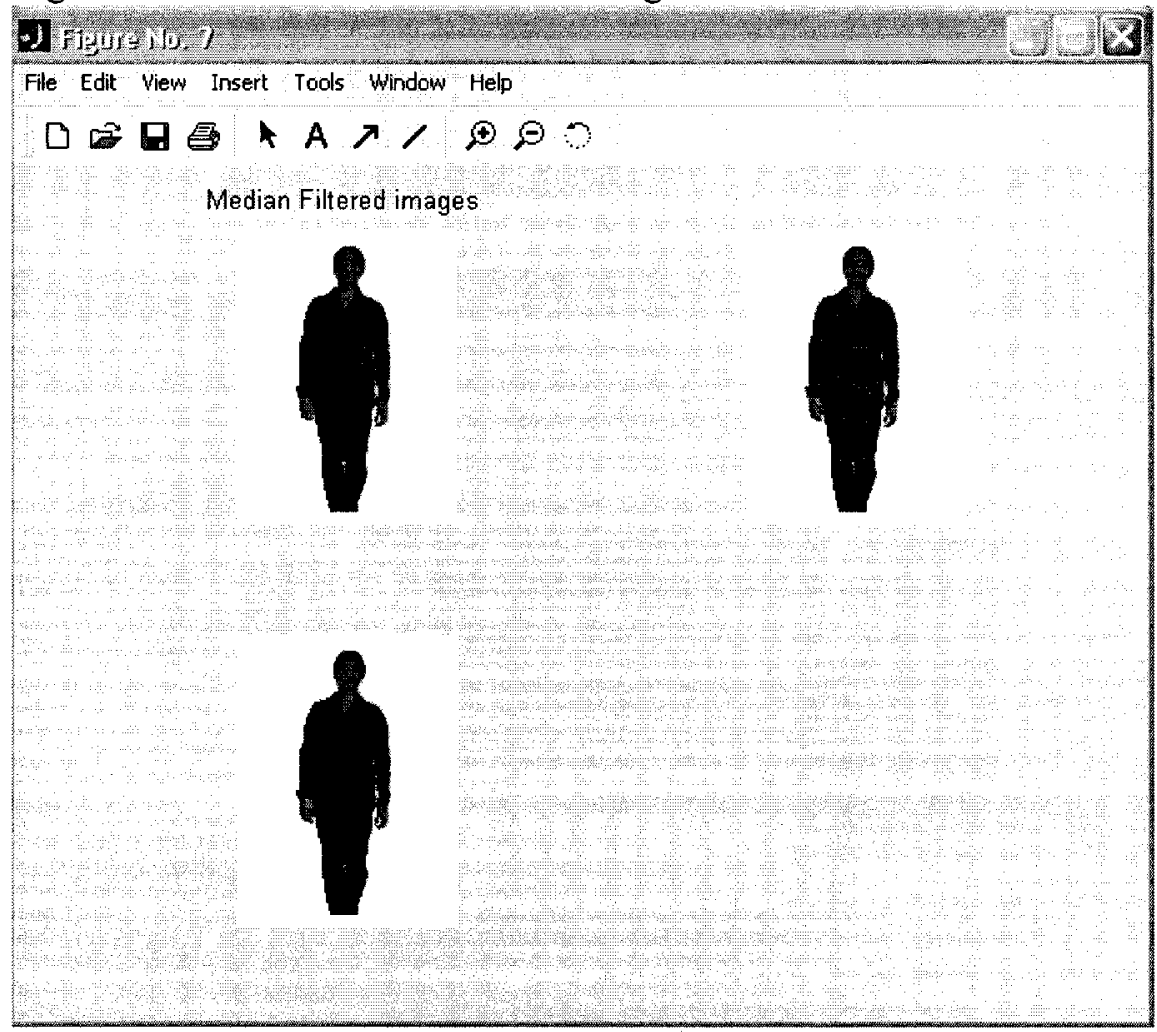

Figure 17: Median Filtered Images 


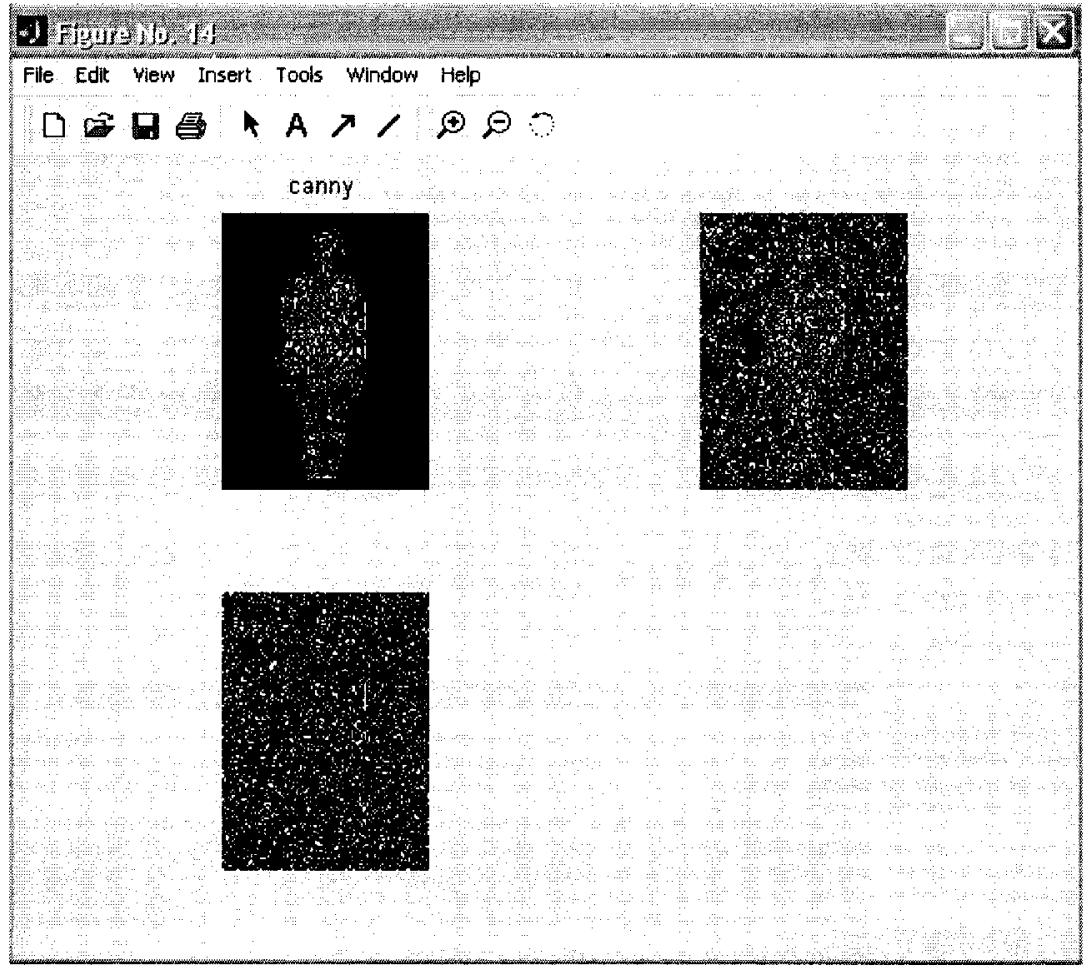

Figure 18: Canny Edge Detector on Original and Gaussian Filtered Gaussian and Salt and Pepper Noisy Images

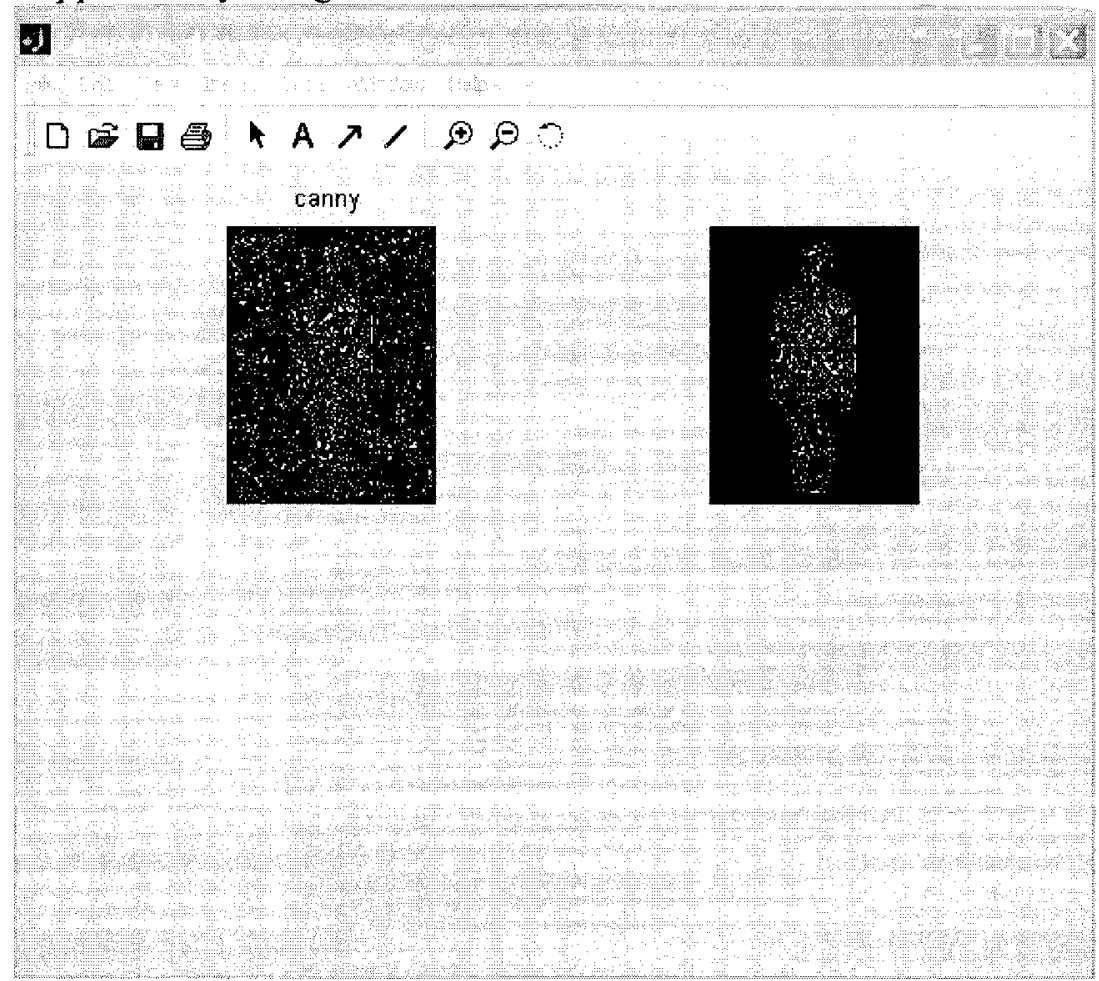

Figure 19: Canny Edge Detector on Median Filtered Gaussian and Salt and Pepper Noisy Images 


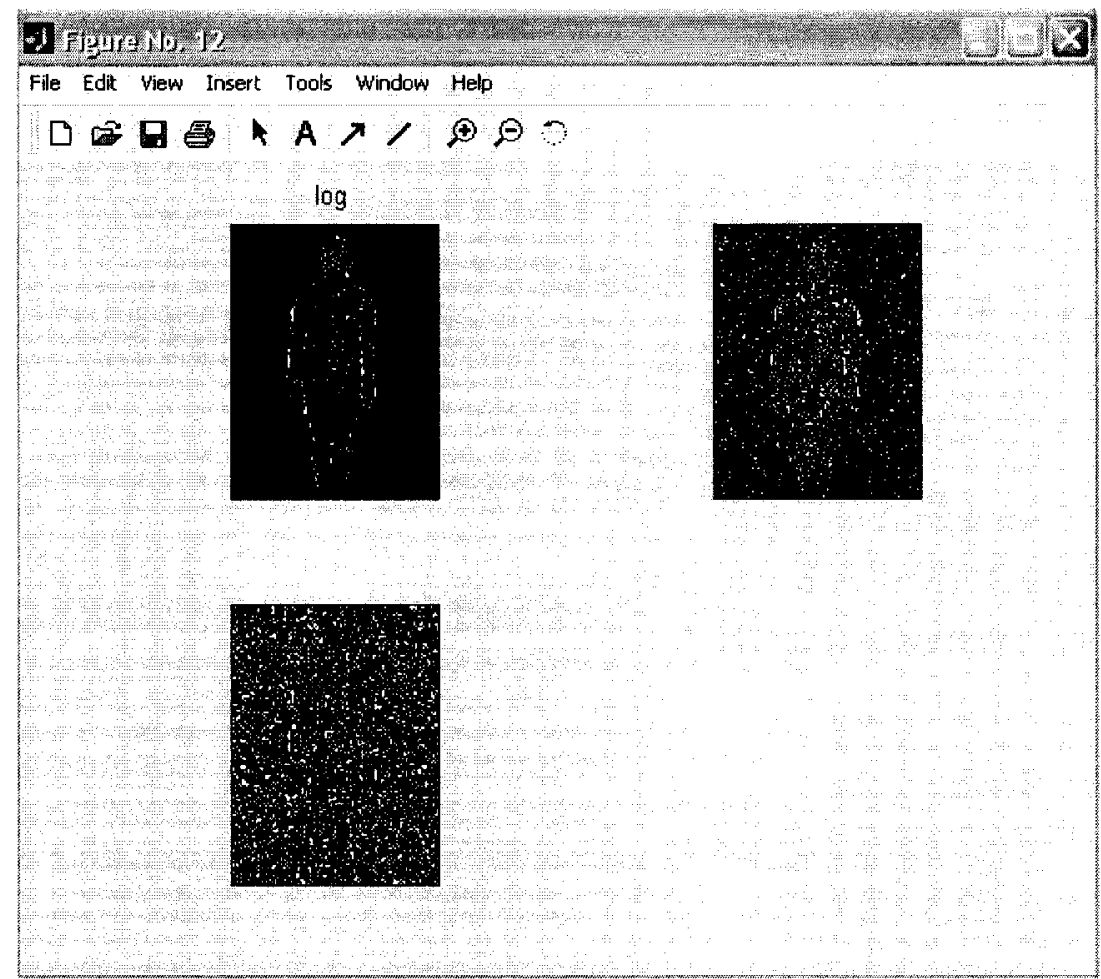

Figure 20: Log Edge Detector on Original and Gaussian Filtered Gaussian and Salt and Pepper Noisy Images

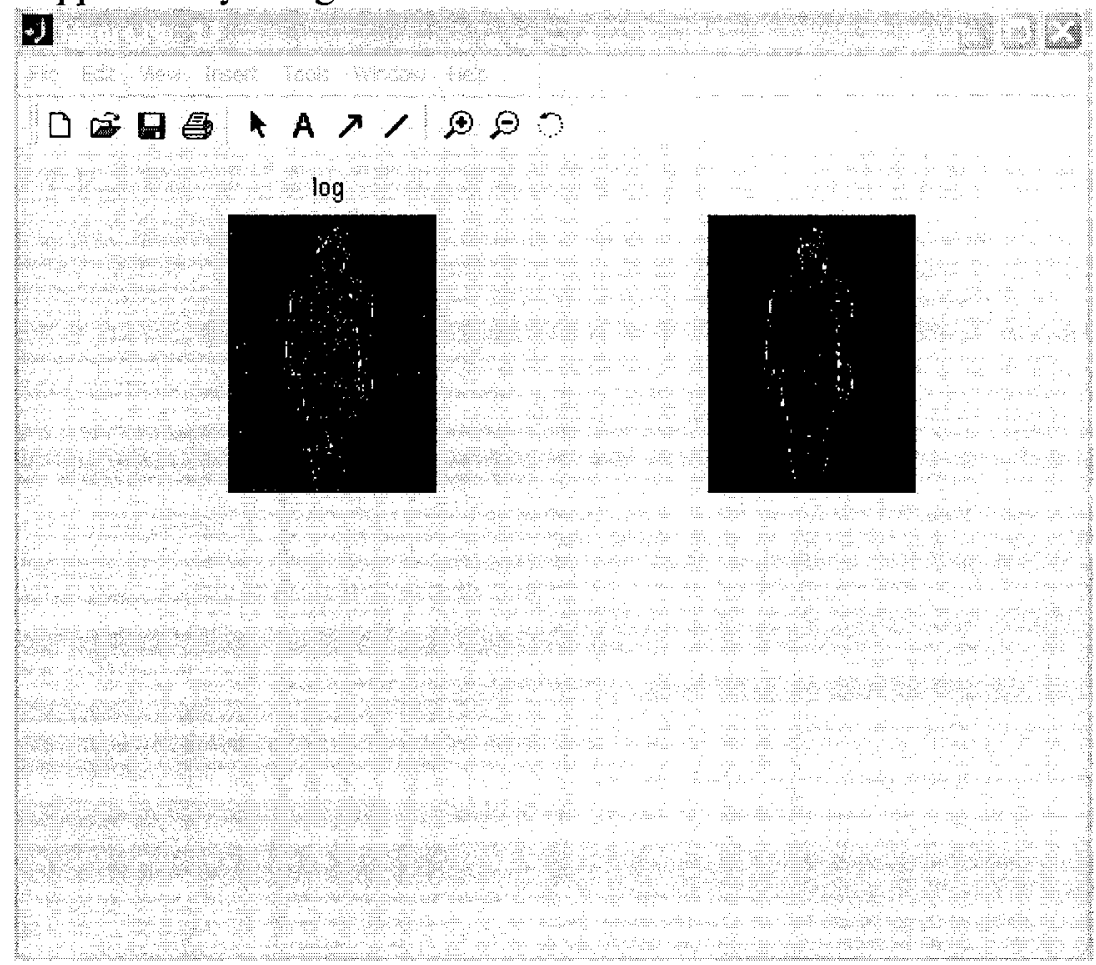

Figure 21: Log Edge Detector on Median Filtered Gaussian and Salt and Pepper Noisy Images 


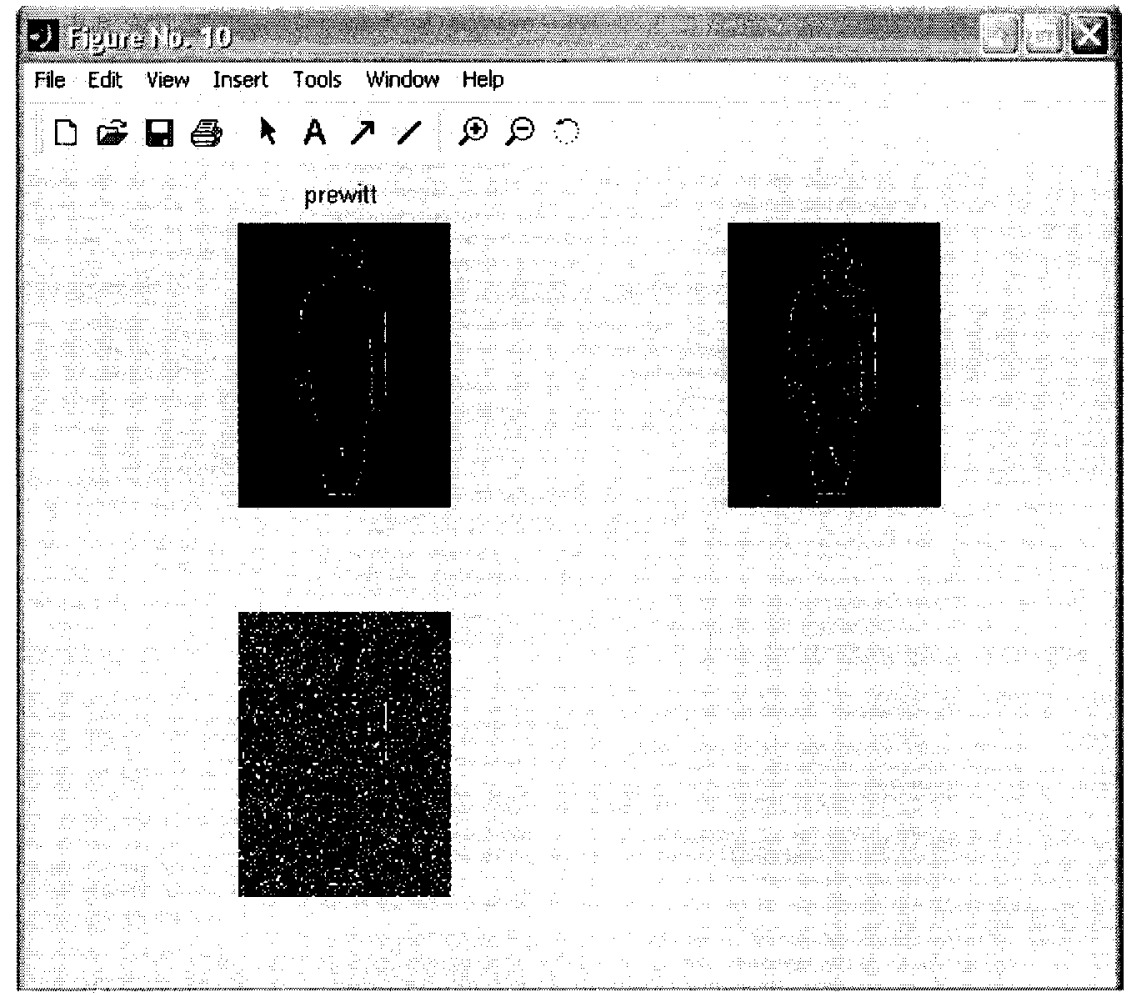

Figure 22: Prewitt Edge Detector on Original and Gaussian Filtered Gaussian and Salt and Pepper Noisy Images

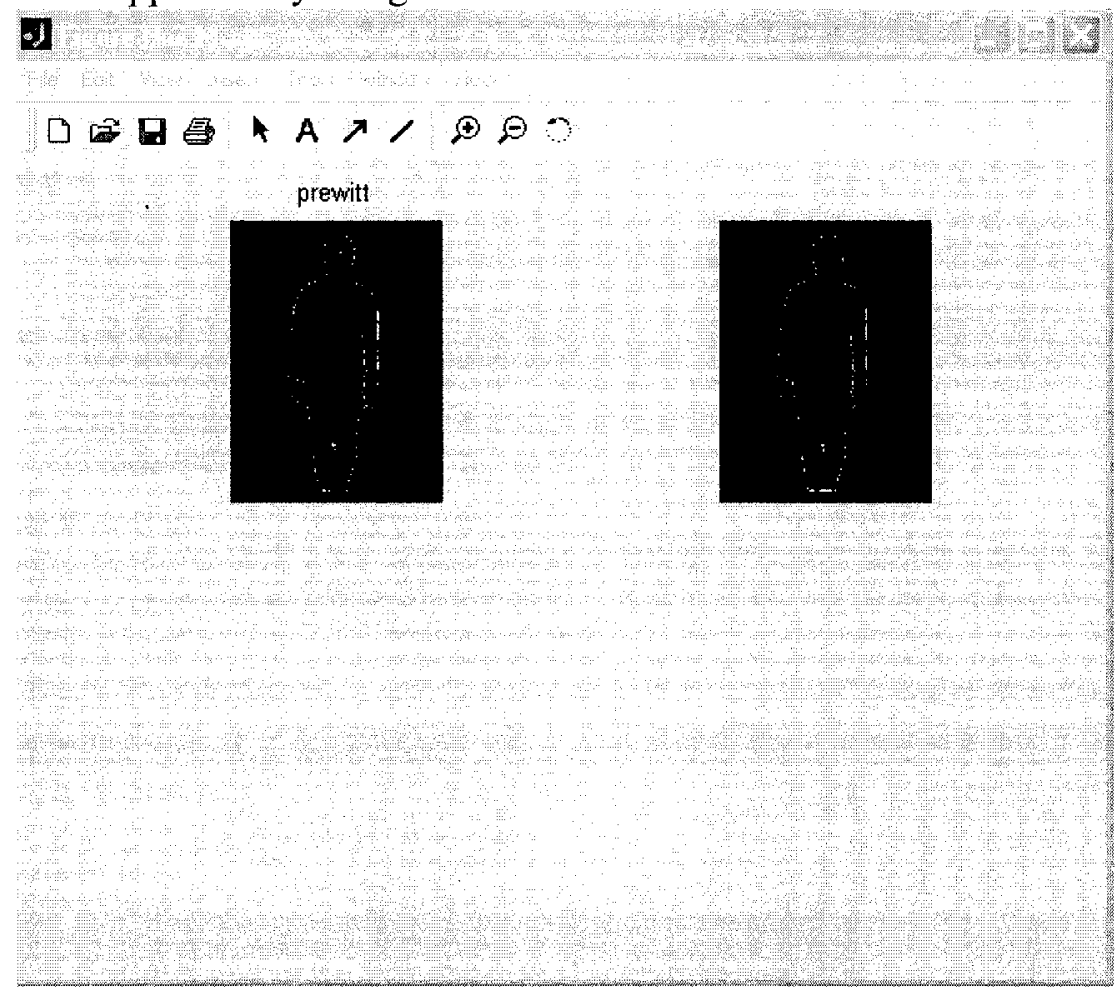

Figure 23: Prewitt Edge Detector on Median Filtered Gaussian and Salt and Pepper Noisy Images 


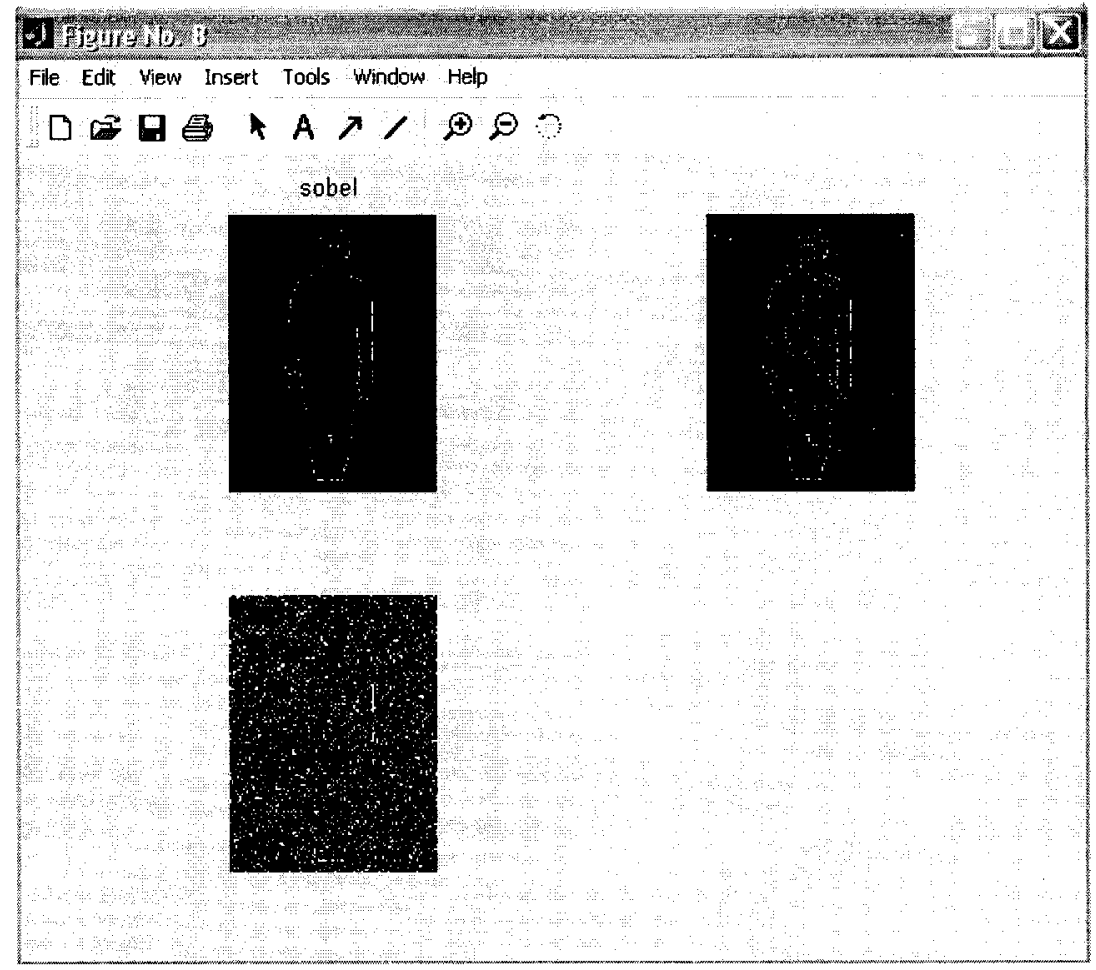

Figure 24: Sobel Edge Detector on Original and Gaussian Filtered Gaussian and Salt and Pepper Noisy Images

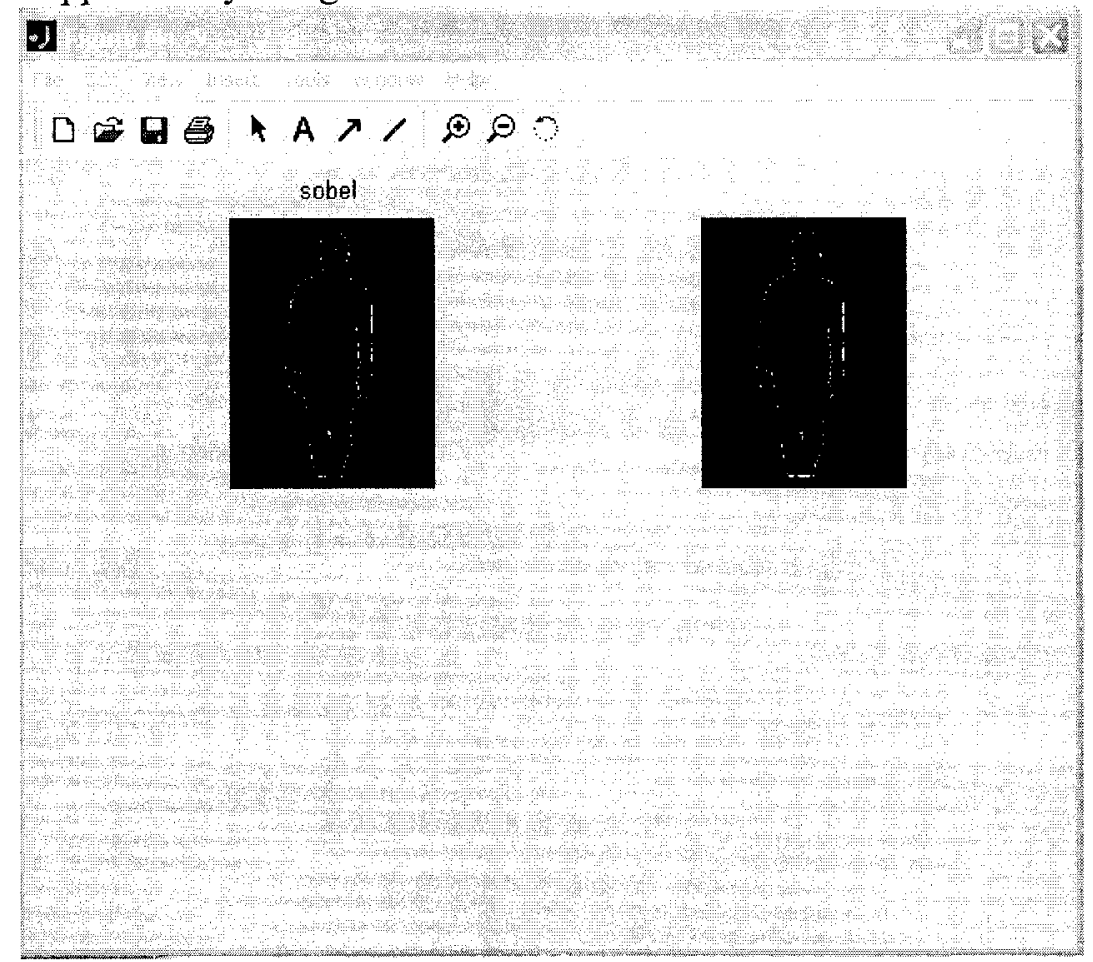

Figure 25: Sobel Edge Detector on Median Filtered Gaussian and Salt and Pepper Noisy Images 